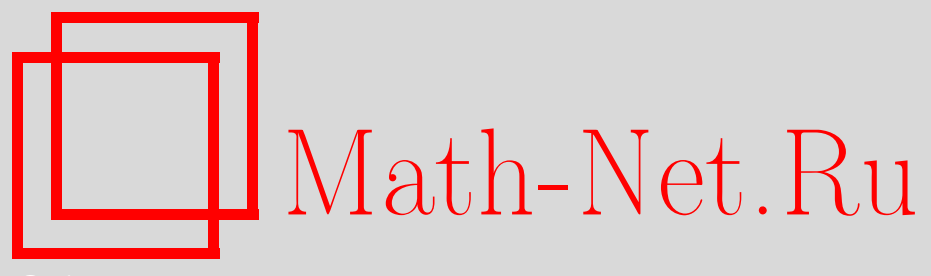

Ан. А. Мучник, Ю. Л. Притыкин, А. Л. Семенов, Последовательности, близкие к периодическим, УМН, 2009, том 64, выпуск 5, 21-96

DOI: https://doi.org/10.4213/rm9315

Использование Общероссийского математического портала Math-Net.Ru подразумевает, что вы прочитали и согласны с пользовательским соглашением http://www . mathnet.ru/rus/agreement

Параметры загрузки:

IP : 54.198 .67 .100

26 апреля 2023 г., 16:44:37

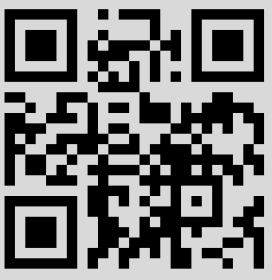




\section{Последовательности, близкие к периодическим}

\section{Ан. А. Мучник, Ю. Л. Притыкин, А. Л. Семенов}

В работе дается обзор понятий и результатов, связанных с обобщениями понятия периодической последовательности, как ставшими классическими, так и новыми. Обсуждаются вопросы, относящиеся к почти периодичности в таких областях, как комбинаторика слов, символическая динамика, выразимость в логических теориях, вычислимость, колмогоровская сложность, теория чисел.

Библиография: 124 названия.

Ключевые слова: комбинаторика слов, символическая динамика, разрешимость логических теорий, почти периодическая последовательность, морфическая последовательность, сложность последовательности, последовательность Туэ-Морса, последовательность Штурма.

\section{СоДЕРЖАНИЕ}

1. Введение............................................ 22

1.1. Первый пример: последовательность Туэ-Морса ............. 24

1.2. Второй пример: повороты окружности, последовательности Штурма и последовательность Фибоначчи ............... 27

1.3. Определения и обозначения.......................... 30

1.4. Почти периодические функции . . . . . . . . . . . . . . . . 31

1.5. Основные классы последовательностей. Регуляторы ......... 32

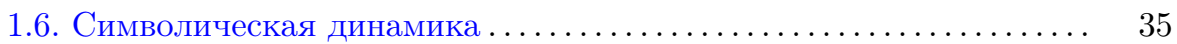

2. Алгебра почти периодических последовательностей ............. 37

2.1. Морфизмы ................................. 37

2.2. Конечно-автоматные преобразования .................. 37

2.3. Блочное произведение........................... 45

2.4. Универсальный метод построения обобщенно почти периодиче-

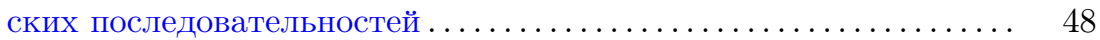

2.5. Произведение последовательностей и почти периодичность при

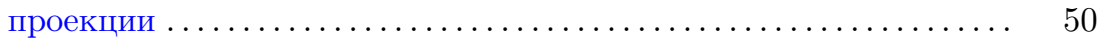

3. Логика почти периодических последовательностей ............... 53

3.1. Логические теории и автоматы ..................... 53

3.2. Разрешимость теорий конкретных последовательностей....... 58

(C) Ан. А. Мучник, Ю. Л. Притыкин, А. Л. Семенов, 2009 
4. Вычислимость для почти периодических последовательностей ..........

4.1. Неразрешимость некоторых свойств почти периодических по-

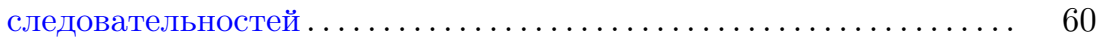

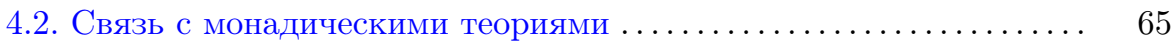

4.3. Нерегулярность некоторых множеств последовательностей .... 66

5. Автоматные и морфические последовательности. Соотношения с почти

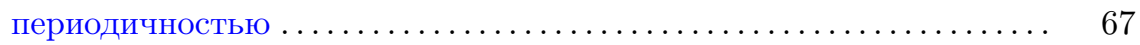

5.1. Автоматные последовательности..................... 67

5.2. Морфические последовательности .................... 69

6. Подслова и сложность почти периодических последовательностей .... 70

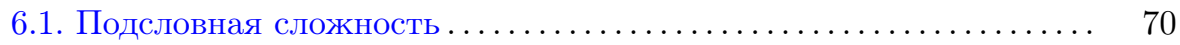

6.2. Нормальные числа и гипотеза Бореля .................. 72

6.3. Последовательности со сложными подсловами ............... 74

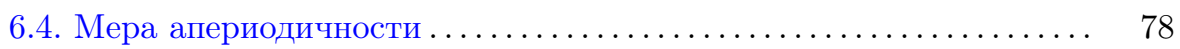

6.5. Предельное поведение регулятора почти периодичности . . . . . 80

7. Близость к периодичности: альтернативные подходы . . . . . . . . . 81

7.1. Квазипериодические последовательности ................. 81

7.2. Разреженные периодичности . . . . . . . . . . . . . . . . 82

7.3. Последовательности Тёплица, периодически альтернируемые морфизмы и последовательность Колакоски ............. 83

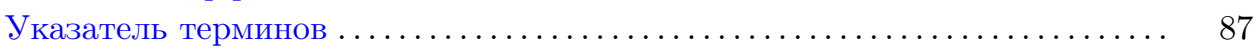

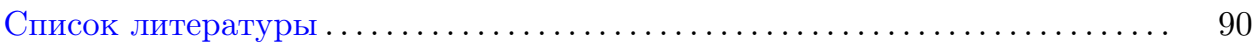

\section{1. Введение}

Наблюдая какой-либо процесс в окружающем мире и пытаясь фиксировать его ход, построить теорию, предсказать, что будет дальше, мы проводим измерения. Эти измерения происходят в дискретные моменты (например, каждый час или каждую наносекунду) и имеют фиксированную точность (один сантиметр или один световой год, или один нанометр). Таким образом, мы сопоставляем процессу последовательность символов (берущихся из конечного алфавита). Сегодня с распространением цифровых технологий и дискретных моделей такое рассмотрение особенно естественно. Математическим отражением этой ситуации является символическая динамика. Рассмотрим (непрерывную) динамическую систему. Разделим пространство состояний системы на конечное количество областей и дадим каждой из областей имя (символ). Посмотрим, в какой из областей окажется система в моменты времени $0,1,2, \ldots$. Получим последовательность символов (символическую траекторию), сопоставленную нашей системе (и исходному состоянию).

Очерченная только что идея символической динамики восходит к XIX веку, к работам Пуанкаре. Многие авторы упоминают в этой связи также Адамара и его публикацию 1898 г. [1], например, Биркгоф говорит о "символах, по существу введенных Адамаром" ([2], взято из [3]). Исследования Адамара были

Работа выполнена при поддержке программы "Ведущие научные школы" (грант НШ-5351.2006.1) и РФФИ (гранты № 05-01-02803, 06-01-00122). 
продолжены Морсом в работе 1921 г. [4]. Однако как самостоятельный предмет символическая динамика впервые начала изучаться полвека назад в работах Морса и Хедлунда 1938-1940 гг. [6], [7], где они установили два важнейших факта символической динамики (верных при естественных ограничениях): 1) символический аналог динамической системы описывает ее точно и 2) символическая траектория обладает некоторым свойством “повторяемости" своих отрезков (точные формулировки см. в п. 1.6). (Тем самым подтверждаются известные максимы - "все повторяется", “ничто не ново под луной” и т. д.) Последовательности, обладающие указанным или другими, аналогичными свойствами - "почти периодические" последовательности - и являются предметом рассмотрения настоящего обзора.

Сто лет назад, в работах [7], [8] важнейший пример почти периодической последовательности был построен Туэ. Это пример последовательности, не содержащей троекратного повторения, подряд, никакого отрезка. Пример Туэ был переоткрыт Морсом в его работе [4]. Туэ подчеркивал важность своего примера и всей области математики, связанной с конечными и бесконечными словами. Сейчас эта область называется комбинаторикой слов. К ней можно отнести ряд классических результатов, среди которых - одно из сложнейших доказательств современной математики - решение Новиковым и Адяном проблемы Бернсайда. (Исходным толчком к возникновению интуиции в решении этой проблемы, по словам Новикова, была последовательность Туэ, переоткрытая советским математиком Аршоном.) Еще один пример замечательного и сложного результата комбинаторики слов - это разрешимость проблемы равенства для систем Туэ с одним соотношением, в котором одна сторона - пустое слово [9].

Вопросы, относящиеся к какой-то символической динамической системе, естественно формулировать на логическом языке, в котором можно говорить о заданной символической последовательности, об операции перехода к следующему моменту времени, в котором есть логические связки и кванторы - по моментам времени и множествам таких моментов. Возникает вопрос о существовании единого алгоритма, отвечающего на все так формулируемые вопросы, иначе говоря, вопрос об алгоритмической разрешимости теории данной системы. Изучая этот вопрос, Семенов рассмотрел символические системы с более общей, чем у Морса, почти периодичностью и установил для них естественно формулируемый критерий разрешимости [10], [11]. Этот результат породил ряд других вопросов алгоритмического и сложностного характера, решенных в [12]-[14].

Прежде чем переходить к более формальному изложению, мы рассмотрим примеры возникающих в различных областях математики "почти периодических" последовательностей (пп. 1.1 и 1.2 и общий контекст символической динамики - п. 1.6). Читатель, интересующийся систематическим изложением основного содержания данного обзора, может сразу перейти к пп. 1.3 и 1.5. Указатель всех основных терминов, встречающихся в обзоре, можно найти на с. 87.

В тексте встречается имеющий единую нумерацию ряд открытых проблем и гипотез, принадлежащих как авторам обзора, так и другим авторам. 
Основное внимание в настоящем обзоре уделяется одномерному случаю, и за рамками обзора остаются различные, часто очень естественные и непосредственные обобщения рассматриваемых понятий на бо́льшие размерности.

Исследования по направлениям, затронутым в настоящем обзоре, естественным образом продолжаются, в частности, они продолжаются на семинарах на мехмате МГУ [15] и в Институте математики в Новосибирске [16].

1.1. Первый пример: последовательность Туэ-Морса. Последовательность Туэ-Морса - одна из самых популярных последовательностей в комбинаторике слов. Она встречается в самых разных сюжетах и служит классическим примером или контрпримером ко многим определениям и утверждениям. Хорошим обзором по свойствам этой последовательности является статья [17] (которую мы во многом используем в изложении этого пункта, вместе с обзором [18] истории комбинаторики слов).

У последовательности Туэ-Морса много различных эквивалентных между собой определений. Давая их, мы будем использовать операцию инвертирования ${ }^{-}$, переводящую 0 в 1 и 1 в 0 . Эта операция распространяется на слова естественным образом. Приведем некоторые из известных определений, пронумеровав.

1) Зададим последовательность $\mathbf{t}=t_{0} t_{1} t_{2} t_{3} t_{4} \ldots$ рекуррентно: $t_{0}=0$ и $t_{2 n}=t_{n}, t_{2 n+1}=\bar{t}_{n}$.

2) Запишем символ: 0. Далее инвертируем его и результат припишем к имеющемуся символу: 01. Теперь имеющийся блок опять инвертируем и припишем к имеющемуся блоку: 0110. Будем продолжать так дальше. Получим последовательность слов:

$$
\begin{aligned}
& u_{0}=0 \\
& u_{1}=01 \\
& u_{2}=0110 \\
& u_{3}=01101001 \\
& u_{4}=0110100110010110 \\
& u_{5}=01101001100101101001011001101001
\end{aligned}
$$

По построению каждое следующее конечное слово начинается с предыдущего. Поэтому все эти слова являются началом некоторого одного (предельного) бесконечного слова:

$$
\mathbf{t}=01101001100101101001011001101001 \ldots
$$

3) Пусть $s_{2}(n)$ обозначает сумму цифр натурального числа $n$, записанного в двоичной системе счисления. Определим двоичную последовательность $\mathbf{t}=$ $t_{0} t_{1} t_{2} t_{3} t_{4} \ldots$ так, что $t_{n}=s_{2}(n)(\bmod 2)$. Обобщением такого определения является понятие автоматной последовательности (см. п. 5.1).

4) Пусть $x$ - переменная. Тогда бесконечное произведение

$$
\prod_{i \geqslant 0}\left(1-x^{2^{i}}\right)=(1-x)\left(1-x^{2}\right)\left(1-x^{4}\right) \cdots=\sum_{i \geqslant 0} f_{i} x
$$


определяет последовательность $f_{i}$. Определим двоичную последовательность $\mathbf{t}=t_{0} t_{1} t_{2} t_{3} t_{4} \ldots$ так, что $(-1)^{t_{i}}=f_{i}$.

Нетрудно доказать, что вышеприведенные определения эквивалентны и задают одну и ту же последовательность, которая называется последовательностъю Туэ-Морса. Есть и другие способы определения. Например, последовательность Туэ-Морса - простейший пример морфической последовательности - неподвижной точки морфизма $0 \rightarrow 01,1 \rightarrow 10$ (см. пп. 5.1, 5.2).

Одним из самых ранних случаев рассмотрения этой последовательности являются работы Туэ: в [7] и [8] он строит эту последовательность как пример бескубной бесконечной последовательности. Здесь кубами мы называем слова вида иии, где $u$ - некоторое непустое слово, а бескубной - последовательность, в которую не входят кубы. Более того, можно доказать, что в $\mathbf{t}$ не входят даже слова вида аиаиа, где $a$ - некоторый символ, а $u$ - некоторое слово. Используя последовательность Туэ-Морса, можно построить бесквадратную (т. е. не содержащую квадратов - слов вида ии для непустого и) последовательность в алфавите из трех символов. В алфавите из двух символов это, как легко видеть, невозможно. Подробнее о работах Туэ см. [19].

Последовательность Туэ-Морса много раз переоткрывали, отсюда и другие имена, которые она иногда носит. Морс переоткрывает эту последовательность в [4] и доказывает, что она почти периодическая, но не периодическая (определения см. в п. 1.5). В [20] голландский шахматист Макс Эйве переоткрывает последовательность Туэ-Морса и использует ее для доказательства существования бесконечной шахматной партии. (Впоследствии для решения этой же задачи ее используют Морс и Хедлунд [21], [22], не зная о работе Эйве.)

Следующую задачу, в которой также появляется последовательность ТуэМорса, можно отнести к теории чисел.

В 1851 г. Пруэ опубликовал заметку на полстранички [23], где, в частности, предложил рассмотреть для каждого числа, начиная с 1, последнюю цифру его суммы цифр. Другими словами - рассмотреть последовательность Пруэ (о последовательностях Пруэ см. также п. 6.4):

$$
0,1,2, \ldots, 9,1,2, \ldots, 9,0,2, \ldots, 9,0,1,3, \ldots
$$

Такая последовательность естественно задает разбиение натуральных чисел на 10 классов (в соответствии с цифрой, стоящей на месте числа). Пруэ, как сказано в его заметке, доказал следующее. Возьмем начальный отрезок натурального ряда, например, длиной миллион (от 0 до 999 999) и найдем сумму чисел каждого класса, попавших в этот отрезок. Тогда все эти суммы для разных классов равны. Более того, равны будут и суммы квадратов этих чисел, суммы кубов, четвертых, пятых степеней. Аналогичная ситуация имеет место, если взять $10^{k}$ вместо миллиона. Тогда и суммы степеней будут совпадать вплоть до $k$-х. Пруэ рассматривал не только степени десяти, а сформулировал общую теорему. В случае двойки (см. наше определение 3)) эта теорема дает следующее. Последовательность Пруэ в этом случае начинается с цифр

$$
0,1,1,0,1,0,0,1,1, \ldots
$$


напишем под ними, для удобства, натуральные числа:

$$
\underline{0}, 1,2, \underline{3}, 4, \underline{5}, \underline{6}, 7,8, \ldots
$$

(места, отвечающие нулям, подчеркнуты). Вот суммы, скажем, для начального отрезка длины 8:

$$
\begin{aligned}
0+3+5+6 & =1+2+4+7=14, \\
0^{2}+3^{2}+5^{2}+6^{2} & =1^{2}+2^{2}+4^{2}+7^{2}=70 .
\end{aligned}
$$

Для того, чтобы получить равенство сумм и кубов тоже, надо рассмотреть начальный отрезок натурального ряда длиной 16, и т. д.

Иначе говоря, задача Пруэ для двойки заключается в следующем. Нужно разделить множество чисел $\left\{0,1,2, \ldots, 2^{N-1}\right\}$ на два множества $I$ и $J$ так, чтобы было выполнено равенство

$$
\sum_{i \in I} i^{k}=\sum_{j \in J} j^{k}
$$

для $k=0,1,2, \ldots, t$.

Пруэ доказал, что это возможно при $N=t+1$. Более точно, по существу Пруэ удалось доказать следующее свойство последовательности t. Определим

$$
\begin{aligned}
& I=\left\{i \in\left\{0,1,2, \ldots, 2^{N-1}\right\}: t_{i}=0\right\}, \\
& J=\left\{j \in\left\{0,1,2, \ldots, 2^{N-1}\right\}: t_{j}=1\right\} .
\end{aligned}
$$

Тогда для всех $0 \leqslant k \leqslant N-1$ выполнено

$$
\sum_{i \in I} i^{k}=\sum_{j \in J} j^{k} .
$$

Задачу Пруэ, через 50 лет после него, рассмотрели Тарри и Эскотт [24]- [27]. Видимо, заметка Пруэ [23] - это самое раннее (хотя и неявное) рассмотрение последовательности Туэ-Морса.

Следующий теоретико-числовой вопрос о последовательности Туэ-Морса был задан Гельфондом в 1968 г. [28]. Четвертое определение из сформулированных выше задает последовательность $f_{i}=(-1)^{t_{i}}$. В силу первого определения $\mathbf{t}$ для любого $i$ выполнено равенство, $f_{2 i}+f_{2 i+1}=0$, поэтому $\sum_{i=0}^{2 N} f_{i}=0$ и $\sum_{i=0}^{2 N+1} f_{i}=f_{2 N+1}$ для любого $N$. Отсюда $\left|\sum_{i=0}^{N} f_{i}\right| \leqslant 1$ для любого $N$. Ввиду соответствия с третьим определением (на основе суммы цифр в двоичном представлении), это значит, что среди натуральных чисел "почти поровну" тех, у которых сумма цифр четна, и тех, у которых сумма цифр нечетна. Гельфонд спросил, верно ли, что аналогичная ситуация имеет место для суммы цифр у простых чисел в двоичной системе счисления. Недавно в [29] было показано, что $\left|\sum_{p \leqslant x} f_{p}\right|=o(\pi(x))$ при $x \rightarrow \infty$, где сумма берется по простым числам $p$, а $\pi(x)$ равно количеству простых чисел, не превосходящих $x$. В связи с этим было бы интересно понять, является ли последовательность $\mathbf{t}_{p}-$ подпоследовательность последовательности $\mathbf{t}$, где $p$ пробегает множество простых чисел почти периодической. 
Последовательность Туэ-Морса встречается также в решении знаменитой проблемы Бернсайда: верно ли, что каждая конечно порожденная группа, удовлетворяющая тождеству $x^{n}=1$, конечна? Как показали Новиков и Адян [30]-[32], бесконечная группа $\Gamma(m, n)$ с $m$ порождающими и с тождеством $x^{n}=1$ существует для всех $m>1$ и для всех нечетных $n \geqslant 4381$. Изучению свойств бесконечных групп $\Gamma(m, n)$ на основе усовершенствования методов из [30]-[32] посвящена монография Адяна [33], где, в частности, оценка 4381 улучшена до 655 .

Один из самых первых шагов в решении проблемы Бернсайда состоит в построении бескубной последовательности в алфавите из двух символов, при этом Новиков и Адян ссылаются на работу Аршона, в которой построена такая последовательность.

Советский математик Соломон Ефимович Аршон родился в 1892 г. С 1934 г. по июнь 1938 г. он был главным редактором Главной редакции технико-теоретической литературы, входившей в состав Объединенного научно-технического издательства Народного комиссариата тяжелой промышленности СССР, по существу С. Е. Аршон руководил основным в стране издательством математической литературы. В конце 1938 - начале 1939 г. он был арестован и погиб (см. [34], [35]). В работе [36] (которая потом фактически повторяется в более известной и часто цитируемой работе [37]) Аршон говорит об открытом вопросе Хинчина 1933 г., поставленном на конференции НИИММ (видимо, НИИ математики и механики механико-математического факультета МГУ), о существовании $n$-буквенной бесквадратной последовательности (т. е. не знает о вышеупомянутых работах Туэ). В качестве положительного ответа Аршон предлагает определенный метод построения $n$-символьной последовательности. Для $n=2$ последовательность Аршона совпадает с последовательностью Туэ-Морса. Для $n \geqslant 3$ последовательности Аршона бесквадратные (он называет это свойство асимметричностью).

\section{2. Второй пример: повороты окружности, последовательности} Штурма и последовательность Фибоначчи. Следующий естественный пример относится к символической динамике. Рассмотрим окружность единичной длины, разделенную на несколько дуг, помеченных буквами конечного алфавита. Выберем число $\alpha$ и отметим какую-то начальную точку $\xi_{0}$ на окружности. Пусть $f$ - отображение окружности в себя, заключающееся в сдвиге всех точек на $\alpha$ в положительном направлении (поворот против часовой стрелки). Рассмотрим траекторию точки $\xi_{0}$ под действием этого отображения, т. е. последовательность точек окружности $\xi_{0}, \xi_{1}=f\left(\xi_{0}\right), \xi_{2}=f\left(f\left(\xi_{0}\right)\right)$, $\xi_{3}=f\left(f\left(f\left(\xi_{0}\right)\right)\right), \ldots$. По траектории получим символическую последовательность: для каждой точки траектории запишем название дуги, в которую она попала (точка может оказаться на границе дуг; в этом случае будем записывать название одной из дуг, скажем, следующей по направлению движения). Последовательности символов, получаемые таким образом, называются вращательными (см. рис. 1).

Ясно, что для рационального $\alpha$ траектория точки, а значит, и соответствующая вращательная последовательность, будет периодической, поскольку длина окружности равна 1. Более интересна ситуация с иррациональным $\alpha-$ в этом 


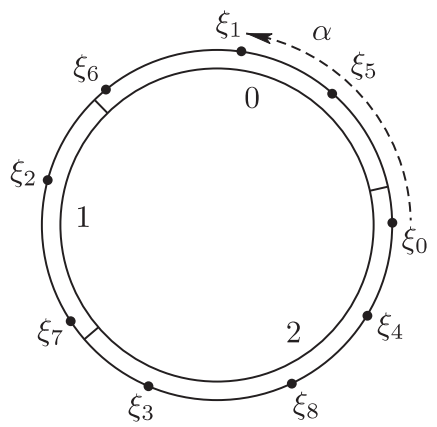

Рис. 1. Вращательная последовательность $x=201220012 \ldots$

случае последовательность не будет периодической, но будет близка к периодической (в некотором, основном для нас, смысле).

Рассмотрим теперь следующий частный случай вращательных последовательностей. Для действительных чисел $\alpha$ и $\rho$ таких, что $0 \leqslant \alpha \leqslant 1$ и $0 \leqslant$ $\rho<1$, определим последовательность $s_{\alpha, \rho}$ как вращательную с начальной точкой $\xi_{0}=\rho$, дугами $(0,1-\alpha)$ и $(1-\alpha, 1)$ и поворотом на $\alpha$. Таким образом, $s_{\alpha, \rho}(n)=0$ при $0 \leqslant\{\alpha n+\rho\}<1-\alpha$ и $s_{\alpha, \rho}(n)=1$ при $1-\alpha \leqslant$ $\{\alpha n+\rho\}<1$ (здесь $\{\cdot\}$ обозначает дробную часть числа). Иначе говоря, $s_{\alpha, \rho}(n)=\lfloor\alpha(n+1)+\rho\rfloor-\lfloor\alpha n+\rho\rfloor$. Такая последовательность называется механической с наклоном $\alpha$ и сдвигом $\rho$.

Термин "механическая последовательность" (используемый в [38], видимо, впервые) для нас отражает простой детерминированный характер процесса порождения последовательности - например, с помощью "механических" поворотов окружности. У этих последовательностей имеется и геометрическая интерпретация (рис. 2). Она получается следующим образом. Чтобы получить механическую последовательность $s_{\alpha, \rho}$, нужно провести прямую $y=\alpha x+\rho$, после чего на каждой вертикальной прямой $x=n$ отметить самые близкие снизу к прямой целые точки. Далее точки под прямой нужно соединить ломаной, она и определяет механическую последовательность: горизонтальное звено ломаной соответствует 0 , а наклоненное под углом $45^{\circ}$ соответствует 1 . Механическая последовательность называется рациональной или иррациональной в зависимости от рациональности наклона $\alpha$. (Определенные так последовательности называются также нижними механическими, в отличие от верхних механических, соответствующих приближениям к прямой сверху, подробно см. в [38].)

Можно рассмотреть другие варианты получения символической последовательности из прямой, нарисованной на клетчатой бумаге. Например, можно отметить все пересечения прямой с линиями сетки, и при пересечении горизонтальной линии писать в последовательность 0, а при пересечении вертикальной писать 1. Другой вариант: можно приближать прямую сверху ломаной, составленной не из горизонтальных и наклонных отрезков, а из горизонтальных и вертикальных: при этом горизонтальный отрезок будет соответствовать 0 , а вертикальный 1. Оба только что названных варианта (как и некоторые 


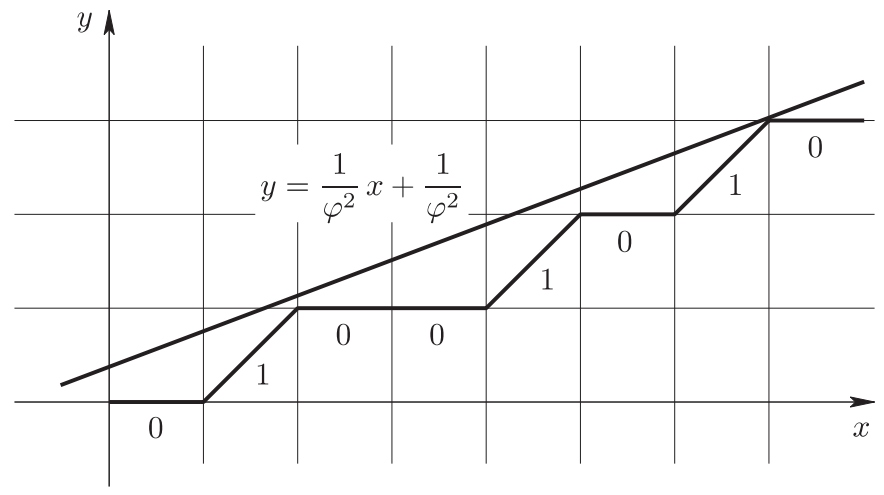

Рис. 2. Механическая последовательность $s_{1 / \varphi^{2}, 1 / \varphi^{2}}=\mathbf{f}$

другие не названные) определяют по-прежнему класс механических последовательностей, причем от параметров этих вариантов к параметрам исходного определения можно переходить вычислимо, и наоборот.

Механические последовательности - один из способов определить знаменитые последовательности Штурма. Первые шаги в изучении последовательностей Штурма можно проследить в работах Бернулли [39]. Эти исследования были продолжены Кристоффелем [40] и Марковым [41]. Однако подробно последовательности Штурма были изучены Морсом и Хедлундом в [6]. Как сообщается в [18], они названы в честь родившегося в Женеве математика Франсуа Штурма (1803-1855), преподававшего в École Polytechnique в Париже с 1840 г. Штурм известен методом решения алгебраических уравнений с помощью рассмотрения перемен знаков соответствующих многочленов. Его имя было использовано Морсом и Хедлундом в связи с нулями решений дифференциального уравнения второго порядка

$$
y^{\prime \prime}+g(x) y=0,
$$

где $g(x)$ - некоторая функция с периодом 1 . Если $k_{n}$ - количество нулей какого-то решения этого уравнения на интервале $[n, n+1)$, то последовательность $a b^{k_{0}} a b^{k_{1}} \ldots$ является последовательностью Штурма (или заключительно периодической). При этом Штурм никогда собственно последовательностями своего имени не занимался. Подробнее о последовательностях Штурма можно прочитать, например, в [42], [38]. Алгоритмические вопросы в связи с последовательностями Штурма рассмотрены в п. 3.2.

Самым известным примером последовательности Штурма является последователъность Фибоначчи $\mathbf{f}=s_{1 / \varphi^{2}, 1 / \varphi^{2}}$, где $\varphi=(1+\sqrt{5}) / 2$ - золотое сечение. Она изображена на рис. 2.

Последовательность $\mathbf{f}$ - наравне с последовательностью Туэ-Морса - служит примером ко многим утверждениям и обладает самыми разными экстремальными свойствами. Некоторые из них мы встретим далее в этой статье.

Другой способ определения последовательности Фибоначчи, которым и объясняется ее название (так как он похож на способ построения знаменитой числовой последовательности Фибоначчи $0,1,1,2,3,5,8,13, \ldots$, в которой каждый 
член равен сумме двух предыдущих), следующий. Положим $u_{0}=0, u_{1}=01$, и далее по правилу $u_{n+2}=u_{n+1} u_{n}$ (мы, так сказать, "складываем" два предыдущих слова, чтобы получить одно последующее). Получим

$$
\begin{aligned}
& u_{0}=0, \\
& u_{1}=01, \\
& u_{2}=010, \\
& u_{3}=01001, \\
& u_{4}=01001010, \\
& u_{5}=0100101001001, \\
& \ldots \ldots \ldots \ldots \ldots \ldots
\end{aligned}
$$

По построению каждое следующее слово здесь начинается с предыдущего, поэтому можно корректно определить последовательность, началами которой являются все слова $u_{n}$ :

$$
\mathbf{f}=010010100100101001010 \ldots
$$

По-другому, последовательность Фибоначчи - чисто морфическая, начинающаяся с 0 и полученная из морфизма $0 \rightarrow 01,1 \rightarrow 0$, см. пп. 5.1, 5.2.

1.3. Определения и обозначения. Введем некоторые основные понятия и обозначения.

Aлфавитом мы называем произвольное конечное множество. Обычно алфавиты мы обозначаем $A, B$ и т. п. Элементы алфавита мы называем буквами или символами. Стандартный алфавит из двух символов $\{0,1\}$ обозначим $\mathbb{B}$. Мы рассматриваем по умолчанию только конечные алфавиты, а при рассмотрении бесконечных алфавитов оговариваем это особо. Для конечного множества $X$ через $\# X$ или $|X|$ обозначим количество элементов в нем. Множество натуральных чисел $\{0,1,2, \ldots\}$ обозначим через $\mathbb{N}$.

Будем рассматривать последовательности над $A$ - отображения $x: \mathbb{N} \rightarrow A$. Определим канторову метрику на последовательностях как $d_{C}(x, y)=2^{-n}$, где $n=\min \{k: x(k) \neq y(k)\}$. Множество всех последовательностей над $A$, наделенное метрикой $d_{C}$, образует стандартное канторовское компактное метрическое пространство, которое мы обозначаем $A^{\mathbb{N}}$.

Таким образом, $\lim _{n \rightarrow \infty} u_{n}=x$, если для любого $i$ существует $n$ такое, что для любого $m>n$ выполнено равенство $u_{m}(i)=x(i)$. Последнее определение годится и для пределов последовательностей слов. Иногда последовательности называют также бесконечными словами или сверхсловами.

Конечная последовательность букв в алфавите $A$ называется еще словом над или в $A$. Через $A^{*}$ обозначим множество всех слов над $A$, включая пустое слово $\Lambda$. Через $|u|$ будем обозначать длину слова $u$, через $|u|_{a}$ - количество букв $a$ в слове $u$. Если $i \leqslant j$ натуральные, через $[i, j]$ обозначим отрезок натурального ряда с концами в $i$ и $j$, т. е. множество $\{i, i+1, i+2, \ldots, j\}$. Отрезок $[0, n]$ будем обозначать просто $[n]$. Через $x[i, j]$ обозначим отрезок последовательности или слова $x-$ слово $x(i) x(i+1) \ldots x(j)$. Говорят, что $[i, j]-$ 
вхождение в $x$ слова $u \in A^{*}$, если $x[i, j]=u$. Слово $u \neq \Lambda$ называется фбактором или подсловом $x$, если $u$ входит в $x$. Множество всевозможных факторов $x$ будем обозначать $\operatorname{Fac}(x)$, множество всевозможных факторов длины $m-$ $\mathrm{Fac}_{m}(x)$. Факторы вида $x[0, i]$ называются префиксами $x$, последовательности вида $x(i) x(i+1) x(i+2) \ldots-$ суфбиксами $x$ и обозначаются $x[i, \infty)$. Мы представляем себе последовательность расположенной горизонтально и идущей слева направо до бесконечности, поэтому будем использовать выражения “левее" и "правее" для меньших и бо́льших индексов соответственно.

Пусть $A, B$ - конечные алфавиты. Отображение $\varphi: A^{*} \rightarrow B^{*}$ называется морфизмом, если для любых $u, v \in A^{*}$ выполнено $\varphi(u v)=\varphi(u) \varphi(v)$. Ясно, что морфизм полностью определяется своими значениями на однобуквенных словах. Морфизм нестирающий, если $|\varphi(a)| \geqslant 1$ для всех $a \in A$. Морфизм называется $k$-равномерным, если $|\varphi(a)|=k$ для всех $a \in A$. 1-равномерный морфизм называется кодированием. Если $x$ - последовательность букв алфавита $A$, по определению положим

$$
\varphi(x)=\varphi(x(0)) \varphi(x(1)) \varphi(x(2)) \cdots
$$

Для действительного числа $x$ обозначим через $\lfloor x\rfloor$ максимальное целое число, не превосходящее $x$, и через $\lceil x\rceil$ минимальное целое число, не меньшее $x$.

1.4. Почти периодические функции. Будем рассматривать функции, определенные на некотором подмножестве $M$ действительных чисел. Пусть $d(f, g)$ - отображение, сопоставляющее паре функций неотрицательное действительное число, причем $d(f, f)=0$. Это отображение будем называть мерой близости. Будем говорить, что подмножество луча $[0,+\infty)$ имеет ограниченные пропуски, если длина отрезков, лежащих в $[0,+\infty)$, где нет его элементов, ограничена. Функция $f$ почти периодическая относителъно $d$, если для любого $\varepsilon>0$ множество таких положительных $T$, для которых $d(f(x), f(x+T))<\varepsilon$, имеет ограниченные пропуски. Ясно, что периодические функции являются почти периодическими при любом $d$.

Рассмотрим случай $M=\mathbb{R}$. В качестве меры близости функций $d(g, h)$ можно брать норму разности $\|f-g\|$ для различных норм $\|\cdot\|$ : равномерной нормы

$$
\|f\|_{\infty}=\sup _{x}|f(x)|
$$

(получаем почти периодические по Бору функции [43]), нормы Безиковича

$$
\|f\|_{B, p}=\limsup _{x \rightarrow \infty}\left(\frac{1}{2 x} \int_{-x}^{x}|f(x)|^{p} d x\right)^{1 / p}
$$

(получаем почти периодические по Безиковичу функции [44]) и многих других.

Чтобы получить случай последовательностей над конечным алфавитом, можно рассмотреть $M=\mathbb{N}$. Конечный алфавит, в котором принимают значения последовательности, можно отождествить с произвольным конечным подмножеством $\mathbb{R}$. Естественно договориться, что последовательности в $k$-буквенном алфавите принимают значения в множестве $\{0,1,2, \ldots, k-1\}$. После этого остается только уточнить, какую меру близости $d(x, y)$ рассматривать. 
Одной из естественных мер близости на множестве последовательностей является канторова метрика. Используя ее в определении выше, мы получаем определение почти периодической последовательности.

Из других естественных функций $d$, которые можно взять в этом определении, рассмотрим дискретный аналог расстояния Безиковича: $d_{B}(x, y)=$ $\liminf _{n \rightarrow \infty} n^{-1} \#\{i: 0 \leqslant i \leqslant n-1, x(i) \neq y(i)\}$. Такая функция уже не будет метрикой, потому что возможно $d_{B}(x, y)=0$ при $x \neq y$. Почти периодические относительно $d_{B}$ последовательности будем называть почти периодическими по Безиковичу. Это определение, введенное в [5] (под другим названием там такие последовательности назывались почти периодическими, а те, что мы в настоящем обзоре называем почти периодическими, назывались рекуррентными), является дискретным вариантом определения почти периодических по Безиковичу функций. Мы обращаемся к этому определению в несколько ином контексте, когда говорим о мере апериодичности последовательности (см. п. 6.4).

1.5. Основные классы последовательностей. Регуляторы. Последовательность $x$ периодическая, если для некоторого $T \in \mathbb{N}$, называемого периодом, имеем $x(i)=x(i+T)$ для любого $i \in \mathbb{N}$. В соответствии с общепринятым соглашением, периодом мы называем также и слово $x[0, T-1]$. Будем называть последовательность заключительно периодической, если $x(i)=x(i+T)$ выполнено для всех $i$, начиная с некоторого $K$. Тогда $x[0, K-1]$ называется предпериодом. Предпериодом будем называть также и число $K$. Последовательность, не являющаяся заключительно периодической, называется апериодической. Множество всех периодических последовательностей обозначим $\mathscr{P}$, множество заключительно периодических обозначим $\mathscr{E} \mathscr{P}$ (eventually periodic). Рассмотрим некоторые расширения этих классов.

В качестве меры близости для последовательностей обычно рассматривается канторова метрика. В соответствии с определением почти периодической функции из п. 1.4 получаем понятие почти периодической последовательности, которое можно сформулировать следующим образом (без упоминания близости).

Последовательность называется почти периодической, если для любого ее подслова найдется число $\Delta$ такое, что во всяком отрезке последовательности, имеющем длину $\Delta$, найдется вхождение этого подслова.

Тем самым, любое слово, входящее в почти периодическую последовательность, входит в нее бесконечное количество раз. Через $\mathscr{A} \mathscr{P}$ (almost periodic) будем обозначать класс всех таких последовательностей. Ясно, что для проверки почти периодичности достаточно убедиться в повторяемости с ограниченными интервалами всех префиксов, а не всех факторов. Почти периодические последовательности называют также равномерно рекуррентными или минимальными.

Будем называть последовательность $x$ заключительно почти периодической, если некоторый ее суффикс почти периодичен. Класс всех таких последовательностей обозначим $\mathscr{E} \mathscr{A} \mathscr{P}$ (eventually almost periodic). 
Если $x \in \mathscr{E} \mathscr{A} \mathscr{P}$, то минимальное такое $n$, что $x[n, \infty) \in \mathscr{A} \mathscr{P}$, будем называть минимальным префиксом и обозначать $\operatorname{pr}(x)$. Заметим, что для любого $m \geqslant$ $\operatorname{pr}(x)$ имеем $x[m, \infty) \in \mathscr{A} \mathscr{P}$.

Последовательность $x$ называется обобщенно почти периодической, если для каждого ее фактора $u$, входящего в нее бесконечное число раз, найдется такое натуральное $l$, что на каждом отрезке длины $l$ последовательности $x$ найдется вхождение слова $u$. Класс всех таких последовательностей обозначим через $\mathscr{G} \mathscr{A} \mathscr{P}$ (generalized almost periodic).

Назовем последовательность $x$ рекуррентной, если каждое слово, которое в нее входит, обязательно входит бесконечное количество раз. Ясно, что если последовательность рекуррентная и обобщенно почти периодическая, то она почти периодическая. Класс рекуррентных последовательностей будем обозначать $\mathscr{R}$. Последовательность заключительно рекуррентная, если некоторый ее суффикс рекуррентен. Класс таких последовательностей обозначим $\mathscr{E} \mathscr{R}$.

В дальнейших рассмотрениях мы будем использовать явное соответствие между длиной фактора (подслова) последовательности и длиной отрезков последовательности, на которых обязательно этот фактор встретится. Соответственно, мы вводим следующее определение.

Регулятором почти периодичности последовательности $x$ называется функция $\mathrm{R}_{x}$, сопоставляющая каждому натуральному $n$ такое минимальное натуральное $\Delta$, что всякое входящее в $x$ слово длины $n$ входит или только в начальный отрезок $x$ длины $\Delta$, или в любой отрезок $x$ длины $\Delta$.

Как видно из определения, регулятор почти периодичности было бы правильнее называть "регулятором обобщенной почти периодичности", однако фактически в основном для нас случае почти периодичности он выполняет функцию "регулятора почти периодичности", и мы сохраняем более короткий термин.

Если говорить более точно, регулятор почти периодичности объединяет в себе две функции: одна следит за расстояниями между вхождениями слов, входящих бесконечное количество раз, а другая следит за тем префиксом, которым ограничивается вхождение слов, входящих конечное количество раз. Иногда стоит эти функции разделять явно, хотя на протяжении настоящей статьи мы этого делать не будем. Часто вместо регулятора нам будет достаточно рассматривать только какую-то верхнюю оценку на него, т. е. функцию $f$ такую, что $f(n) \geqslant \mathrm{R}_{x}(n)$ для всех $n$ (коротко мы пишем $f \geqslant \mathrm{R}_{x}$ ).

Будем говорить, что последовательность $x$ эфбективно обобщенно почти периодическал, если $x$ вычислима, обобщенно почти периодична, и некоторая оценка сверху на регулятор почти периодичности последовательности $x$ также вычислима. Мы получим эквивалентное определение, если заменим требование вычислимости некоторой оценки сверху на регулятор требованием вычислимости регулятора в точности.

ПрЕДЛОЖЕНИЕ 1.5.1. Пусть последователъность $x \in \mathscr{G} \mathscr{A} \mathscr{P}$ вычислима, и вычислима некоторая функция $f \geqslant \mathrm{R}_{x}$. Тогда функиия $\mathrm{R}_{x}$ вычислима.

ДокАЗАТЕЛЬСтво. Слова длины $n$, входящие в последовательность $x$ бесконечное число раз, - это в точности слова длины $n$, входящие в $x[f(n), f(2 n)]$, и мы можем их выписать, поскольку $x$ вычислима. Точно также мы можем 
выписать слова длины $n$, входящие в $x$ конечное число раз и входящие хотя бы один раз.

Теперь нетрудно перебором найти границу $l_{1}$, не превышающую $f(n)$, на которой заканчиваются концы всех слов длины $n$, имеющих только конечное число вхождений в $x$.

Аналогично предыдущему, можно найти множество $K$ слов длины $f(n)$, хотя бы один раз входящих в $x$. Теперь достаточно найти минимальное $l_{2}$ такое, что каждое подслово длины $n$, встречающееся в $x$ бесконечно много раз, встречается в каждом подслове длины $l_{2}$ каждого из слов множества $K$. Тогда любое подслово $x$ длины $n$, встречающееся в $x$ бесконечно много раз, входит в любое подслово $x$ длины $l_{2}$, и заменить $l_{2}$ на меньшее нельзя.

Таким образом, $\mathrm{R}_{x}(n)=\max \left(l_{1}, l_{2}\right)$. Предложение доказано.

Назовем последовательность эффективно почти периодической, если она почти периодична и эффективно обобщенно почти периодична. Определение эффективной почти периодичности можно упростить.

ПРЕДЛОЖЕНИЕ 1.5.2. Почти периодическая последовательность $x$ эфбективно почти периодична тогда и только тогда, когда х вычислима и множество подслов $\operatorname{Fac}(x)$ разрешимо.

ДоказАтельство. $\Rightarrow$. Пусть $x \in \mathscr{A} \mathscr{P}$ вычислима, и мы умеем вычислять некоторую оценку $f \geqslant \mathrm{R}_{x}$. Тогда чтобы найти все слова длины $n$ в $x$, достаточно взять произвольное слово длины $f(n)$ в $x$ : множество его подслов длины $n$ в точности является множеством подслов длины $n$ всей последовательности $x$, в соответствии с определением почти периодичности.

$\Leftarrow$. Пусть $x \in \mathscr{A} \mathscr{P}$ вычислима и множество $\operatorname{Fac}(x)$ разрешимо. Чтобы найти значение $\mathrm{R}_{x}(n)$, будем перебирать все натуральные числа по очереди, начиная, скажем, с $n$. Проверяя число $m$, мы смотрим, верно ли, что каждое из слов множества $\mathrm{Fac}_{m}(x)$ (подслова $x$ длины $m$ ) содержит все слова из $\operatorname{Fac}_{n}(x)$ (подслова $x$ длины $n$ ). Если это так, то $\mathrm{R}_{x}(n) \leqslant m$, а иначе $\mathrm{R}_{x}(n)>m$. Поскольку $x \in \mathscr{A} \mathscr{P}$, мы когда-нибудь найдем подходящее $m$.

Предложение доказано.

Докажем, что последовательность Туэ-Морса

$$
\mathbf{t}=0110100110010110 \ldots
$$

(см. п. 1.1) почти периодична. Это следует из теоремы 2.4.1, но мы приведем здесь рассуждение явно. Заметим, что достаточно доказать, что для каждого $n$ найдется такое $l_{n}$, что на каждом отрезке длины $l_{n}$ в $\mathbf{t}$ найдется вхождение слова $u_{n}$ (в обозначениях второго определения из раздела 1.1).

В силу второго определения $\mathbf{t}$ имеем $u_{n+1}=u_{n} \overline{u_{n}}$. Поэтому $\mathbf{t}$ есть бесконечное произведение сомножителей вида $u_{n} \overline{u_{n}}$ и $\overline{u_{n}} u_{n}$, каждый из которых содержит $u_{n}$. Поскольку $\left|u_{n}\right|=2^{n}$, то длины указанных сомножителей равны $2^{n+1}$, и на любом отрезке последовательности $\mathbf{t}$ длины $2^{n+2}$ есть вхождение $u_{n}$.

Назовем последовательность точно почти периодической, если каждое слово, которое в нее входит, входит в некоторой арифметической прогрессии. Более формально, $x$ - точно почти периодическая, если для любого входящего 
в нее слова $u$ найдутся такие $a, d \in \mathbb{N}$, что $x[a+i d, a+i d+|u|-1]=u$ для всех $i \geqslant 0$. При этом $u$ может входить и где угодно еще в последовательности - мы не требуем, чтобы все вхождения слова $u$ образовывали арифметическую прогрессию. Ясно, что любая точно почти периодическая последовательность почти периодична. Класс точно почти периодических последовательностей обозначим $\mathscr{P} \mathscr{A} \mathscr{P}$. На с. 76 описывается арифметический метод построения последовательностей (использованный в доказательстве теоремы 6.3.2), с помощью которого можно строить точно почти периодические последовательности.

Несложно видеть, что $\mathscr{P} \subset \mathscr{A} \mathscr{P} \subset \mathscr{E} \mathscr{A} \mathscr{P} \subset \mathscr{G} \mathscr{A} \mathscr{P}$. Оказывается, все эти включения строгие. Например, известная последовательность Туэ-Морса пример последовательности из $\mathscr{A} \mathscr{P}$, но не из $\mathscr{P}$. Более того, класс $\mathscr{A} \mathscr{P}$ континуален, тогда как $\mathscr{P}$ счетный, доказательство см. в [45] или [46]. Неравенство $\mathscr{A} \mathscr{P} \subsetneq \mathscr{E} \mathscr{A} \mathscr{P}$ очевидно (можно взять $10000 \ldots \in \mathscr{E} \mathscr{A} \mathscr{P} \backslash \mathscr{A} \mathscr{P}$ ). Неравенство $\mathscr{E} \mathscr{A} \mathscr{P} \subsetneq \mathscr{G} \mathscr{A} \mathscr{P}$ было доказано в [47] (см. также в [12]). Кроме того, можно заметить следующие включения $\mathscr{P} \subset \mathscr{E} \mathscr{P} \subset \mathscr{E} \mathscr{A} \mathscr{P}$ и $\mathscr{P} \subset \mathscr{P} \mathscr{A} \mathscr{P} \subset \mathscr{A} \mathscr{P}$, все из которых тоже, очевидно, строгие. Также $\mathscr{A} \mathscr{P} \cap \mathscr{E} \mathscr{P}=$ Per.

Введенные классы изображены на рис. 3.

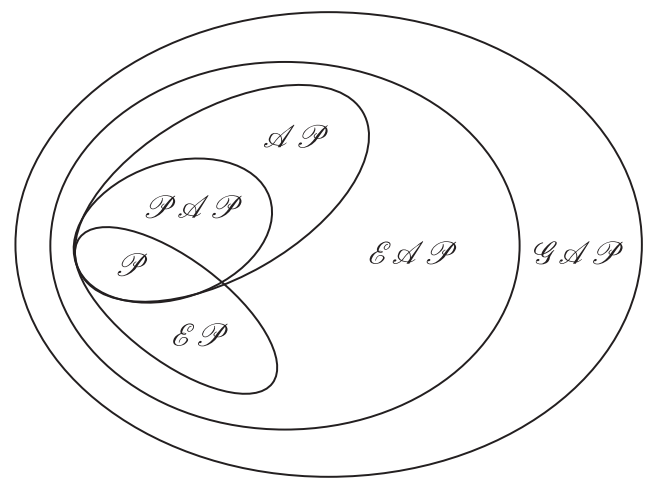

Рис. 3. Классы последовательностей

1.6. Символическая динамика. Изначально понятие почти периодичности возникло в связи с символической динамикой. Топологическая динамическая система - это топологическое пространство $V$ с заданным на нем непрерывным отображением $f: V \rightarrow V$. Пусть $V, f$ - топологическая динамическая система, $A_{1}, \ldots, A_{k}$ - попарно непересекающиеся открытые подмножества $V$ и $x_{0}$ - точка, орбита которой $\left\{f^{n}\left(x_{0}\right): n \in \mathbb{N}\right\}$ лежит в $\bigcup_{i=1}^{k} A_{i}$. Определим последовательность $x: \mathbb{N} \rightarrow\{1, \ldots, k\}$ условием $f^{n}\left(x_{0}\right) \in A_{x(n)}$. Таким образом, (некоторым) точкам пространства $V$ мы сопоставили последовательности букв алфавита $A=\{1, \ldots, k\}$. При этом применению отображения $f$ соответствует операция левого сдвига $L$ : $L\left(a_{0} a_{1} a_{2} a_{3} \ldots\right)=a_{1} a_{2} a_{3} \ldots{ }^{1}$.

\footnotetext{
${ }^{1}$ Условие $\left\{f^{n}\left(x_{0}\right): n \in \mathbb{N}\right\} \subseteq \bigcup_{i=1}^{k} A_{i}$ во многих интересных случаях не выполняется, как, например, в случае с поворотами окружности (см. п. 1.2). Точки траектории могут попадать на границы областей, но обычно можно единым универсальным способом разрешать эту ситуацию и выбирать, какой символ писать в последовательность.
} 
Ясно, что периодические последовательности описывают только самые простые ситуации. Однако оказывается, что в довольно широком классе ситуаций возникающая символическая последовательность будет обладать свойствами, близкими к свойствам периодической последовательности. Это наблюдение можно формализовывать разными способами, примеры точных результатов такого типа - теоремы 1.6.1-1.6.3 ниже.

ТЕОРема 1.6.1. Если пространство V компактно и орбита каждой точки плотна в $V$, то последовательность $x$ почти периодическая.

Более того, несложно показать, что каждая почти периодическая последовательность может быть получена таким образом, как описано в теореме 1.6.1. Для этого надо взять пространство всех последовательностей с топологией, порожденной метрикой $d_{C}$, для каждой буквы $a$ в качестве области $A_{a}$ взять множество последовательностей, начинающихся с $a$, и в качестве отображения $f$ взять отображение $L$ левого сдвига, а в качестве $V$ взять замыкание $x$ относительно действия $f$, после чего замкнуть получившееся множество относительно метрики $d_{C}$. Подробнее см., например, [38].

Следующий факт о траекториях в метрических пространствах - частный случай теоремы 1.6.1.

Теорема 1.6.2. Если $V$ - компактное метрическое пространство, и $f$ изометрия, то последовательность х почти периодическая.

Теоремы 1.6.1 и 1.6.2 в том или ином виде уже можно считать фольклором (доказательства можно найти, например, в [46]). Видимо, это уже не совсем так для результата теоремы 1.6.3.

Пусть $T^{s}-s$-мерный тор $[0,1)^{s}$ с естественной метрикой, индуцированной метрикой в $\mathbb{R}^{s}$. Множество в $\mathbb{R}^{s}$ называется алгебраическим, если оно является решением системы (строгих или нестрогих) полиномиальных неравенств с целыми коэффициентами. Множество в $\mathbb{R}^{s}$ называется полуалгебраическим, если оно является объединением алгебраических множеств. Множество в $T^{s}$ полуалгебраическое, если оно является пересечением полуалгебраического множества в $\mathbb{R}^{s}$ с тором $T^{s}$.

ТЕОРема 1.6.3 [47]. Пусть $V$ - s-мерный тор, $x_{0}$ - алгебраическая точка в $V, f$ - сдвиг на вектор с алгебрачческими координатами, все $A_{i}$ - полуалгебрачческие. Тогда последовательность х эффективно почти периодическая.

Из доказательства теоремы 1.6.3 в [46] легко можно получить и хорошо известный неэффективный результат: если $V-s$-мерный тор, $x_{0}$ - произвольная точка в $V, f$ - сдвиг на произвольный вектор, то последовательность $x$ почти периодическая. Отсюда следует, что все вращательные последовательности, и в частности, последовательности Штурма (см. п. 1.2), являются почти периодическими.

Один из синонимов почти периодической последовательности - минимальная последовательность. Почти периодические последовательности действительно обладают следующим свойством минимальности: они имеют минимальное множество подслов среди всех последовательностей, в следующем смысле. 
ПРЕДЛОЖЕНИЕ 1.6.4. 1) Для любых бесконечных последовательностей $x$ и у если $x$ почти периодическая $и \operatorname{Fac}(y) \subseteq \operatorname{Fac}(x), \operatorname{mo} \operatorname{Fac}(y)=\operatorname{Fac}(x)$.

2) Для любой последовательности у существует почти периодическая последовательность $x$ такая, что $\operatorname{Fac}(x) \subseteq \operatorname{Fac}(y)$.

Доказательство предложения 1.6.4 можно найти, например, в [48].

\section{2. Алгебра почти периодических последовательностей}

2.1. Морфизмы. Пусть $\varphi: A^{*} \rightarrow B^{*}$ - морфизм, $x$ - обобщенно почти периодическая последовательность над алфавитом $A$. В [46] было показано, что если последовательность $\varphi(x)$ бесконечна, то она обобщенно почти периодична. Заметим, что для $x$ - почти периодической $\varphi(x)$ будет почти периодической (если бесконечна). Действительно, достаточно показать, что для любого слова $u=x[i, j]$ слово $\varphi(u)=\varphi(x(i)) \ldots \varphi(x(j))$ встречается в $\varphi(x)$ бесконечно много раз. Но это следует из определения $\varphi(x)$ и из того, что $x$ почти периодична, и значит, слово $u$ встречается в ней бесконечно много раз. Очевидно, для $x$ - заключительно почти периодической $\varphi(x)$ будет снова заключительно почти периодической (если она бесконечна). Несложно видеть, что классы эффективно обобщенно почти периодических и эффективно почти периодических последовательностей также замкнуты относительно действий морфизмов.

2.2. Конечно-автоматные преобразования. Представляется интересным рассматривать преобразования последовательностей и пытаться понять, сохраняют ли эти преобразования свойства почти периодичности. Простейшими алгоритмическими преобразованиями можно считать конечно-автоматные. В то же время конечно-автоматные преобразования можно воспринимать как наиболее широкий класс преобразований, сохраняющих определенную алгебраическую структуру последовательностей, в частности, обобщающих морфизмы.

Для различных классов последовательностей известны результаты о замкнутости относительно конечно-автоматных преобразований - например, см. теоремы 5.1.3 и 5.2.3. В настоящем пункте мы обсуждаем замкнутость относительно таких преобразований последовательностей с различными свойствами типа почти периодичности.

Другая мотивация заключается в связи конечно-автоматных преобразований (а точнее, распознающих автоматов на последовательностях) с монадическими теориями на натуральных числах с отношением порядка, которым посвящен п. 3.1 (см. теорему 3.1.5).

Конечно-автоматным преобразователем назовем совокупность $M=\langle A, B$, $Q, \tilde{q}, \lambda, \mu\rangle$, где $A$ и $B$ - конечные множества, называемые соответственно входной и выходной алфавит, $Q$ - конечное множество состояний, $\tilde{q} \in Q$ - выделенное состояние, называемое начальным, $\lambda: Q \times A \rightarrow B^{*}-$ функция выхода, $\mu: Q \times A \rightarrow Q-$ функция переходов. Пусть $x \in A^{\mathbb{N}}$. Последовательность $\left(p_{n}\right)_{n=0}^{\infty}$ элементов множества $Q$ назовем ходом преобразователя $M$ на $x$, если $p_{0}=\tilde{q}$ и для каждого $n$ выполняется $p_{n+1}=\mu\left(p_{n}, x(n)\right)$. Последовательность $M(x)$, определяемую как бесконечная конкатенация слов $\lambda\left(p_{0}, x(0)\right), \lambda\left(p_{1}, x(1)\right)$, 
$\lambda\left(p_{2}, x(2)\right), \ldots$, где $\left(p_{n}\right)_{n=0}^{\infty}$ - ход преобразователя $M$ на $x$, назовем образом последовательности $x$ под действием $M$.

Если для каждых $a \in A, q \in Q$ выполнено $|\lambda(q, a)|=1$, то преобразователь $M$ называется равномерным. Применение произвольного конечно-автоматного преобразователя к последовательности можно представить как последовательное применение равномерного конечно-автоматного преобразователя и некоторого морфизма.

ПРеДЛОЖЕНИЕ 2.2.1. Пусть $M=\langle A, B, Q, \tilde{q}, \lambda, \mu\rangle-$ конечно-автоматный преобразователь, $x$ - последовательность. Тогда существует такой равномерный конечно-автоматный преобразователь $M^{\prime}$ и такой морфизм $\varphi$, что $M(x)=\varphi\left(M^{\prime}(x)\right)$.

ДокАЗАТЕЛЬСтво. Положим $M^{\prime}=\left\langle A, Q \times A, Q, \tilde{q}, \lambda^{\prime}, \mu\right\rangle$, так что $\lambda^{\prime}(q, a)=$ $\langle q, a\rangle$ для любых $q \in Q$ и $a \in A$. Определим также морфизм $\varphi: Q \times A \rightarrow B$, так что $\varphi(\langle q, a\rangle)=\lambda(q, a)$ для любых $q \in Q$ и $a \in A$. Ясно тогда, что $M(x)=$ $\varphi\left(M^{\prime}(x)\right)$. Предложение доказано.

Поэтому часто для упрощения ситуации мы ограничиваемся рассмотрением равномерных конечно-автоматных преобразователей. Если $[i, j]$ - вхождение слова $u$ в последовательность $x$, причем $p_{i}=q$, где $\left(p_{n}\right)_{n=0}^{\infty}-$ ход преобразователя $M$ на $x$, то будем говорить, что преобразователь $M$ nодxодum к этому вхождению слова $u$ в состоянии $q$.

По существу, следующая теорема доказана в [11] (см. также [46]). Приведем ее с полным доказательством (по [46]).

Для произвольной функции $g$ обозначим $g \circ g \circ \cdots \circ g\left(m\right.$ раз) через $g^{m}$.

Теорема 2.2.2 [11], [46]. Пусть $M$ - конечно-автоматный преобразователь с т состояниями и $x \in \mathscr{G} \mathscr{A} \mathscr{P}$. Тогда верно следующее.

1) $M(x) \in \mathscr{G} \mathscr{A} \mathscr{P}$.

2) Пусть $M$ - равномерный преобразователь. Тогда $\mathrm{R}_{M(x)}(n) \leqslant h(h(n))$ для всех $n$, где $h(n)=g^{m}(n)-1 u g(n)=\mathrm{R}_{x}(n)+1$.

3) Если х эббективно обобщенно почти периодическая, то $M(x)$ такэе эфбективно обобщенно почти периодическая.

Лемма 2.2.3. Пусть $M$ - равномерный конечно-автоматный преобразователь с $m$ состояниями и $x \in \mathscr{G} \mathscr{A} \mathscr{P}$. Пусть $v=M(x)[i, j]-$ вхождение слова длины $n$ в $M(x)$ такое, что $i \geqslant h(n)$, где $h(t)=g^{m}(t)-1, g(t)=\mathrm{R}_{x}(t)+1$. Тогда найдется такое $r$, что $j-h(n) \leqslant r \leqslant i-1 u M(x)[r, r+n-1]=v$.

ДокАЗАтельство. Будем считать сначала, что $v=M(x)[i, j]$ - просто достаточно далекое от начала вхождение слова $v$ в $M(x)$. Объясним, как найти искомое вхождение $v$, которое обозначим $M(x)[r, s]$. При этом мы будем делать разные допущения, которые подытожим и точно сформулируем позднее.

Итак, пусть $v=M(x)[i, j]$ и $u_{1}=x[i, j]$ - прообраз $v$ в $x$. Пусть преобразователь $M$ подходит к позиции $i$, находясь в состоянии $q_{1}$. Если $i$ достаточно велико, то найдется вхождение $u_{1}=x\left[i_{2}, j_{2}\right]$ левее отрезка $[i, j]$, но достаточно близко к нему. Если $M$ подходит к $i_{2}$ в состоянии $q_{1}$, то $M(x)\left[i_{2}, j_{2}\right]=v$. Иначе $M$ подходит к $i_{2}$ в каком-то состоянии $q_{2} \neq q_{1}$ (рис. 4 ). 


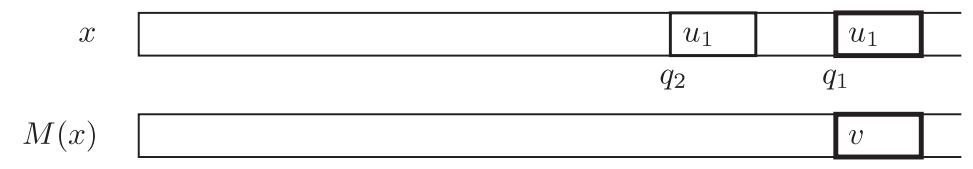

Рис. 4. Иллюстрация к доказательству теоремы 2.2 .2

Положим $u_{2}=x\left[i_{2}, j\right]$. Если $i_{2}$ достаточно велико, то найдется вхождение $u_{2}=x\left[i_{3}, j_{3}\right]$ левее отрезка $\left[i_{2}, j\right]$, но достаточно близко к нему. Если $M$ подходит к $i_{3}$ в состоянии $q_{1}$, то $M(x)\left[i_{3}, i_{3}+n-1\right]=v$, так как $u_{2}$ начинается с $u_{1}$. Если $M$ подходит к $i_{3}$ в состоянии $q_{2}$, то $M(x)\left[i_{3}, j_{3}\right]=M(x)\left[i_{2}, j\right]$, и тогда $M(x)\left[j_{3}-n+1, j_{3}\right]=v$, так как $u_{2}$ заканчивается словом $u_{1}$. В худшем случае $M$ подходит к $i_{3}$ в состоянии $q_{3}$ таком, что $q_{3} \neq q_{2}$ и $q_{3} \neq q_{1}$. При этом положим $u_{3}=x\left[i_{3}, j\right]$ (рис. 5$)$.

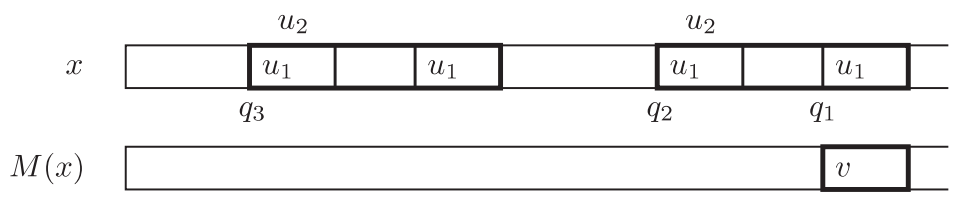

Рис. 5. Иллюстрация к доказательству теоремы 2.2 .2

Рассуждая так дальше, мы, все время выбирая худший случай, найдем такие $i_{2}, \ldots, i_{m+1}$, что к каким-то двум из $i_{1}=i, i_{2}, \ldots, i_{m+1}$ преобразователь $M$ подходит в одинаковых состояниях. Таким образом, слово $v$ обязательно встретится в $M(x)\left[i_{m+1}, j-1\right]$.

Проанализируем теперь вышеприведенное рассуждение. Вначале мы ищем вхождение $u_{1}=x\left[i_{2}, j_{2}\right]$. Это можно сделать, если $i \geqslant \mathrm{R}_{x}(n)$ - исходя из определения обобщенной почти периодичности, это означает, что $u_{1}$ входит в $x$ бесконечно много раз. При этом получится $(j-1)-i_{2}+1 \leqslant \mathrm{R}_{x}(n)$ - этого достаточно, чтобы на отрезке $x\left[i_{2}, j-1\right]$ нашлось вхождение $u_{1}$. Отсюда $i_{2} \geqslant j-\mathrm{R}_{x}(n)$ и $\left|u_{2}\right|=j-i_{2}+1 \leqslant \mathrm{R}_{x}(n)+1$.

Далее аналогично получаем, что при $i_{2} \geqslant \mathrm{R}_{x}\left(\mathrm{R}_{x}(n)+1\right) \geqslant \mathrm{R}_{x}\left(\left|u_{2}\right|\right)$ действительно можно найти вхождение $u_{2}=x\left[i_{3}, j_{3}\right]$, причем $i_{3} \geqslant j-\mathrm{R}_{x}\left(\mathrm{R}_{x}(n)+1\right)$ и $\left|u_{3}\right| \leqslant \mathrm{R}_{x}\left(\mathrm{R}_{x}(n)+1\right)+1$.

Положим $g=\mathrm{R}_{x}+1$. Аналогично рассуждая, получим, что при $i_{m} \geqslant$ $g^{m}(n)-1$ можно выбрать $i_{m+1} \geqslant j-g^{m}(n)+1$. Лемма доказана.

ДоказАТЕЛЬСтво теОремы 2.2.2. Пусть $x \in \mathscr{G} \mathscr{A} \mathscr{P}$ и на $x$ действует конечно-автоматный преобразователь $M$ с $m$ состояниями.

1) Из предложения 2.2.1 следует, что достаточно доказать утверждение только для равномерного $M$, так как морфизмы сохраняют обобщенную почти периодичность. Для равномерного $M$ из леммы 2.2 .3 следует, что если слово $v$ длины $n$ входит в $M(x)$ бесконечно много раз, то оно входит на каждом отрезке длины $g^{m}(n)-1$ в $M(x)$. Поэтому $M(x)$ обобщенно почти периодическая.

2 ) Покажем, как найти оценку сверху на $\mathrm{R}_{M(x)}$. Обозначим $h(n)=g^{m}(n)-1$, где $g(n)=\mathrm{R}_{x}(n)+1$. 
Пусть слово $v$ длины $n$ входит в $M(x)$ бесконечно много раз. Тогда по 1$)$ оно встретится на любом отрезке длины $h(n)$.

Пусть теперь слово $v$ длины $n$ входит в $M(x)$ конечное количество раз. Докажем, что тогда $v$ не входит в $M(x)$ правее позиции $h(h(n))$. Действительно, предположим, что это не так. Тогда на каждом отрезке длины $h(n)$ слова $M(x)[0, h(h(n))-1]$ найдется вхождение $v$, так как по 1) от каждого такого вхождения можно найти еще одно слева не дальше, чем на расстоянии $h(n)$. Но $v$ входит в $M(x)$ лишь конечное количество раз. Поэтому в $M(x)$ найдется слово $w$ длины $h(n)$ (где-то сильно справа), которое не содержит $v$. Тогда всюду слева от него на любом отрезке длины $h(h(n))$ найдется вхождение $w$, значит, и в $M(x)[0, h(h(n))-1]$ тоже. Получаем противоречие, так как на любом отрезке длины $h(n)$ в $M(x)[0, h(h(n))-1]$ есть вхождение $v$, но $w$ не содержит $v$ и входит в $M(x)[0, h(h(n))-1]$.

Таким образом, объединяя утверждения последних двух абзацев, получаем оценку $\mathrm{R}_{M(x)}(n) \leqslant h(h(n))$.

3) Пусть теперь последовательность $x$ эффективно обобщенно почти периодическая, т. е. $x$ вычислима и регулятор $\mathrm{R}_{x}$ вычислим. Аналогично п. 1$)$, достаточно рассматривать только случай равномерного $M$, так как морфизмы сохраняют эффективную обобщенную почти периодичность. Ясно, что зная $M$ и умея вычислять $x$, мы можем вычислять $M(x)$. Пункт 2) позволяет также вычислять оценку сверху на $\mathrm{R}_{M(x)}$. Значит, $M(x)$ эффективно обобщенно почти периодическая. Теорема доказана.

Из теоремы 2.2.2 сразу следует, что образ при конечно-автоматном преобразовании заключительно почти периодической последовательности обобщенно почти периодичен. Но оказывается, можно доказать более сильное утверждение.

ТеОРема 2.2.4 [47], [12]. Пусть $M$ - конечно-автоматный преобразователь. Тогда если $x \in \mathscr{E} \mathscr{A} \mathscr{P}$, то $M(x) \in \mathscr{E} \mathscr{A} \mathscr{P}$.

Доказательство теоремы 2.2.4, приведенное в [47], не эффективно в следующем смысле. Допустим, мы знаем $x \in \mathscr{A} \mathscr{P}$ и регулятор $\mathrm{R}_{x}$. Тогда по теореме 2.2.4 существует оценка сверху на $\operatorname{pr}(M(x))$, так как $M(x) \in \mathscr{E} \mathscr{A} \mathscr{P}$, но доказательство из [47] не позволяет по имеющимся данным найти никакую такую оценку эффективно (и даже до некоторого момента не позволяло надеяться, что такой эффективный способ вообще существует).

Следующий эффективный вариант теоремы 2.2.4 был объявлен в [49] и доказан в [12]. Приведем план доказательства.

ТеОРема 2.2.5 [12]. Пусть $M$ - равномерный конечно-автоматный преобразователь с т состояниями, и $x \in \mathscr{A} \mathscr{P}$. Тогда $M(x) \in \mathscr{E} \mathscr{A} \mathscr{P} u$

$$
\operatorname{pr}(M(x)) \leqslant \mathrm{R}_{x}^{m}(1)+\mathrm{R}_{x}^{m-1}(1)+\cdots+\mathrm{R}_{x}(1) .
$$

Пусть $M=\langle A, B, Q, \tilde{q}, \lambda, \mu\rangle$ - равномерный преобразователь. Доказательство проходит индукцией по количеству состояний преобразователя. Будем считать без ограничения общности, что $B=Q \times A$ и для всех $q \in Q, a \in A$ выполнено $\lambda(q, a)=\langle q, a\rangle$. Действительно, общий случай произвольного $B$ 
и произвольного $\lambda$ получается из рассматриваемого отождествлением каких-то пар $\langle q, a\rangle$ между собой (см. предложение 2.2.1).

База индукции - обратимый конечно-автоматный преобразователь.

Назовем конечно-автоматный преобразователь $M=\langle A, B, Q, \tilde{q}, \lambda, \mu\rangle$ обратимым, если для каждых $q \in Q$ и $a \in A$ существует ровно одно состояние $q^{\prime} \in Q$ такое, что $\mu\left(q^{\prime}, a\right)=q$. Другими словами, в таком преобразователе каждая буква входного алфавита осуществляет взаимно однозначное отображение множества состояний в себя. Находясь в некотором состоянии и зная последовательность предыдущих входных символов, можно восстановить и последовательность пройденных состояний (в этом и заключается свойство обратимости).

ПРЕДЛОЖЕНИЕ 2.2.6 [12]. Пусть $M$ - обратимый равномерный конечноавтоматный преобразователь с $m$ состояниями. Тогда если $x \in \mathscr{A} \mathscr{P}$, то $M(x) \in \mathscr{A} \mathscr{P}$.

ДокАЗАТЕЛЬство. Пусть $v=M(x)[i, j]$ - вхождение слова $v$ длины $n$ в $M(x)$ и $u_{1}=x[i, j]$ - прообраз $v$ в $x$, к которому $M$ подходит в состоянии $q_{1}$. Тогда найдется вхождение $u_{1}=x\left[i_{2}, j_{2}\right]$ такое, что $i_{2}>i$, но $j_{2} \leqslant i+\mathrm{R}_{x}(n)$. Если $M$ подходит к $i_{2}$ в состоянии $q_{1}$, то $M(x)\left[i_{2}, j_{2}\right]=v$. Иначе $M$ подходит к $i_{2}$ в каком-то состоянии $q_{2} \neq q_{1}$.

Положим $u_{2}=x\left[i, j_{2}\right]$. Имеем $\left|u_{2}\right| \leqslant \mathrm{R}_{x}(n)+1$. Тогда найдется вхождение $u_{2}=x\left[i_{3}, j_{3}\right]$ такое, что $i_{3}>i$, но $j_{3} \leqslant i+\mathrm{R}_{x}\left(\mathrm{R}_{x}(n)+1\right)$. Если $M$ подходит к $j_{3}-n+1$ в состоянии $q_{1}$, то $M(x)\left[j_{3}-i+1, j_{3}\right]=v$, так как $u_{2}$ заканчивается словом $u_{1}$. Если $M$ подходит к $j_{3}-n+1$ в состоянии $q_{2}$, то в силу обратимости $M$ подходит к $i_{2}$ в состоянии $q_{1}$, и тогда $M(x)\left[i_{2}, i_{2}+n-1\right]=v$, так как $u_{2}$ начинается с $u_{1}$. В худшем случае $M$ подходит к $j_{3}-n+1$ в состоянии $q_{3}$, таком что $q_{3} \neq q_{1}, q_{3} \neq q_{2}$ (рис. 6 ).

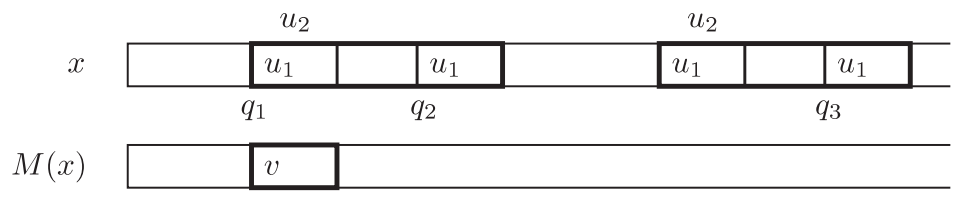

Рис. 6. Иллюстрация к доказательству предложения 2.2 .6

Рассуждая так дальше, построим такие $j_{2}, \ldots, j_{m+1}$, что хотя бы к каким-то двум из позиций $i=j-n+1=j_{1}-n+1, j_{2}-n+1, \ldots, j_{m+1}-n+1$ преобразователь подходит в одинаковых состояниях. При этом $j_{m+1} \leqslant i+g^{m}(n)-1$, где $g=$ $\mathrm{R}_{x}+1$.

Таким образом, каждое слово длины $n$, входящее в $M(x)$ бесконечно много раз, входит на любом отрезке длины $g^{m}(n)-1$. Следовательно, $M(x) \in \mathscr{A} \mathscr{P}$. Предложение доказано.

Видно, что доказательство предложения 2.2.6 очень похоже на доказательство теоремы 2.2.2, приведенное выше, только рассуждение проходит не "справа налево", а "слева направо". Такое "обращение" возможно за счет обратимости преобразователя. 
Для индукционного перехода рассмотрим следующую конструкцию. Пусть $x \in A^{\mathbb{N}}$, и символ $a \in A$ входит в $x$ бесконечное количество раз. Проведем разрез в $x$ после каждого вхождения символа $a$. Тогда $x$ разрежется на блоки вида $u a$, где $u \in(A \backslash\{a\})^{*}$, т. е. на слова, содержащие ровно один символ $a$ на последнем месте. Если символ $a$ встречается в $x$ с ограниченными интервалами, то количество всевозможных таких блоков конечно (например, если $x \in \mathscr{G} \mathscr{A} \mathscr{P}$, то их длины не больше $\left.\mathrm{R}_{x}(1)\right)$. Закодируем однозначно эти блоки буквами некоторого конечного алфавита, обозначим этот алфавит $\mathrm{b}_{a, x}(A)$. Таким образом, мы из $x$ получили новую последовательность в этом алфавите. Последовательность, полученную из нее отбрасыванием первого символа, назовем a-разбиением последовательности $x$ и обозначим $\mathrm{s}_{a}(x)$. Например, 0 -разбиение последовательности 3200122403100110 . . - это (0)(12240)(310)(0)(110) . . .

Несложно доказать, что если $x \in \mathscr{A} \mathscr{P}$ и символ $а$ входит в $x$, то $_{a}(x) \in \mathscr{A} \mathscr{P}$ (см. [12]).

Индукционный переход в доказательстве теоремы 2.2.5 заключается в следующем. Допустим, исходный преобразователь не обратим. Тогда образ множества состояний $Q$ преобразователя под действием какой-то буквы $a$ входного алфавита строго содержится в $Q$. Рассмотрев теперь вместо последовательности ее $a$-разбиение и, считая преобразователь определенным на алфавите $\mathrm{b}_{a, x}(A)$, сведем задачу к самой же себе, но с меньшим количеством состояний в преобразователе. При этом, переходя от последовательности $x$ к $a$-разбиению $\mathrm{s}_{a}(x)$, мы отбрасываем первый символ последовательности блоков, что соответствует отбрасыванию начального блока длины не более $\mathrm{R}_{x}(1)$ от последовательности $x$. Сделав так некоторое количество раз, мы приходим к ситуации, в которой к последовательности применяется обратимый конечно-автоматный преобразователь (преобразователь с одним состоянием всегда обратим). Подробнее доказательство см. в [12].

Примечательно, что, таким образом, мы получаем второе доказательство теоремы 2.2.4.

Заметим, что, формально говоря, введенные нами классы последовательностей - это классы последовательностей над конечным алфавитом. Однако определения почти периодической последовательности, обобщенно почти периодической последовательности, заключительно почти периодической последовательности переносятся в точности и на бесконечный алфавит. Например, континуум различных почти периодических последовательностей над бесконечным алфавитом можно построить с помощью арифметического метода построения последовательностей (см. с. 76); они будут точно почти периодическими. Несложно построить и другие нетривиальные примеры.

На бесконечный алфавит можно обобщить и понятие конечно-автоматного преобразователя. При этом не требуется никакой эффективности, в частности, функции переходов могут быть совершенно произвольными. Все результаты настоящего раздела верны и для конечно-автоматных преобразователей на последовательностях над бесконечным алфавитом (но это, вообще говоря, может быть не так для результатов во всей статье, например, для результатов о разрешимости раздела 3). 
Идея рассмотрения бесконечного алфавита позволяет получить новый результат и для класса последовательностей над конечным алфавитом. Следующее утверждение следует понимать как результат о последовательностях над бесконечным алфавитом. При этом частным случаем будет точно такое же утверждение, подразумеваемое для последовательностей над конечным алфавитом.

ТеОРема 2.2.7. Пусть $M$ - конечно-автоматный преобразователь. Тогда если последовательность $x$ заключительно почти периодическая над бесконечным алфавитом, то $M(x)$ также заключительно почти периодическая (над бесконечным алфавитом).

ПЛАН ДОКАЗАТЕЛЬСТВА. Мы приводим только план доказательства этой теоремы, поскольку доказательство следует схеме доказательства теоремы 2.2.5, изложенной выше, почти без изменений. Единственное важное замечание утверждение нужно доказывать над бесконечным алфавитом, по-прежнему индукцией по количеству состояний преобразователя.

База индукции - обратимый преобразователь. Можно доказать, аналогично предложению 2.2.6, что класс рекуррентных последовательностей (даже и над бесконечным алфавитом) сохраняется под действием обратимых конечно-автоматных преобразователей.

Для шага индукции нужно опять рассмотреть разбиения, описанные выше на с. 42. Можно доказать, что $a$-разбиение рекуррентной последовательности, в которую входит символ $a$, рекуррентно. Однако, как несложно видеть, разбиение рекуррентной последовательности даже и над конечным алфавитом может стать последовательностью над бесконечным алфавитом, потому что в рекуррентной последовательности нет ограничения на расстояние между вхождениями буквы. Теорема доказана.

Ввиду теоремы 2.2.7 естественно спросить следующее.

ПроБЛЕмА 1. Предложить эффективную версию теоремы 2.2.7, которая позволит упростить известные и получить новые результаты о разрешимости монадических теорий одноместных функций (о монадических теориях см. п. 3.1).

Представляется интересным разрабатывать все введенные нами понятия и для бесконечного алфавита.

ПроБЛЕма 2. Провести более систематическое изучение обобщений понятий и результатов, введенных в настоящей статье, на бесконечный алфавит, в том числе, снабженный дополнительной структурой.

ЗАмЕчАниЕ 2.2.8. В обоих случаях в первую очередь, в контексте настоящего обзора, нас могут интересовать вопросы разрешимости логических теорий. Однако можно ожидать и другие интересные результаты.

Следуя [50], [51], будем называть последовательность $x$ линейно почти neриодической ${ }^{2}$, если она почти периодическая и имеет не более чем линейный

\footnotetext{
${ }^{2} \mathrm{~B}$ [50], [51] такие последовательности называются линейно рекуррентными.
} 
регулятор почти периодичности, т. е. $\mathrm{R}_{x}(n) \leqslant C n$ для некоторого $C \in \mathbb{R}, C \geqslant 1$. (Несложно видеть, что при $C<1$ последовательностей $x$ с $\mathrm{R}_{x}(n) \leqslant C n$ для всех $n$ не существует.) Заметим, что класс линейно почти периодических последовательностей также сохраняется при конечно-автоматных преобразованиях с точностью до префикса. Естественно при этом определить класс заключительно линейно почти периодических последовательностей, у которых некоторый суффикс линейно почти периодический.

ТеОРема 2.2.9. Пусть $M$ - конечно-автоматный преобразователь с $m$ состояниями. Тогда если $x$ заключительно линейно почти периодическая, то $M(x)$ такюе заключительно линейно почти периодическая.

Доказательство. Имеем $M(x) \in \mathscr{E} \mathscr{A} \mathscr{P}$ по теореме 2.2.4. Пусть $\mathrm{R}_{x}(n) \leqslant$ $C n$ для всех $n$ и некоторого $C$. Тогда по теореме 2.2 .2$, п. 2) регулятор $\mathrm{R}_{M(x)}(n)$ можно оценить сверху величиной $g^{2 m}(n)$, где $g(n)=C n+1$. Таким образом, $\mathrm{R}_{M(x)}(n) \leqslant C^{2 m} n+C^{2 m-1}+C^{2 m-2}+\cdots+C+1$, что означает, что $M(x)$ заключительно линейно почти периодическая. Теорема доказана.

ПроБлемА 3. Можно ли улучшить оценку $\mathrm{R}_{M(x)}(n) \leqslant C^{2 m} n+\left(C^{2 m}-1\right) \times$ $(C-1)^{-1}$ в теореме 2.2 .9 (см. также проблему 4$)$ ?

Минимальный префикс $\operatorname{pr}(M(x))$ в теореме 2.2.9 также можно оценить, с помощью теоремы 2.2.5.

Теорема 2.2.2, п. 2) дает оценку сверху порядка $\mathrm{R}_{x}^{2 m}$ для регулятора выходной последовательности $M(x)$ при применении равномерного преобразователя $M$ с $m$ состояниями к последовательности $x$. Если воспользоваться общим планом доказательства теоремы 2.2.5 применительно к случаю обобщенно почти периодических последовательностей, то, по всей видимости, эту оценку можно улучшить. Однако, к сожалению, компактной формулы, удобной для формулировки и изложения доказательства, нам найти не удалось, поэтому полностью этот план для обобщенно почти периодических последовательностей мы здесь реализовывать не будем.

Тем не менее обобщение ключевой части этого плана - теоремы 2.2.6 - представляет и самостоятельный интерес. Назовем конечно-автоматный преобразователь $M$ почти обратимым относительно последовательности $x$, если каждая буква $a$, встречающаяся в $x$ бесконечное количество раз, осуществляет взаимно однозначное отображение на множестве состояний преобразователя $M$.

ПРЕДЛОЖЕНИЕ 2.2.10. Пусть $M$ - почти обратимый относительно последовательности $x$ равномерный конечно-автоматный преобразователь $с m$ состояниями, и $x \in \mathscr{G} \mathscr{A} \mathscr{P}$. Тогда $M(x) \in \mathscr{G} \mathscr{A} \mathscr{P}$, причем $\mathrm{R}_{M(x)}(n) \leqslant g^{m}(n)-1$, где $g(n)=\mathrm{R}_{x}(n)+1$.

ДоКАЗАТЕЛЬСТВо. Положим $h(n)=g^{m}(n)-1$ для всех $n$, где $g(n)=$ $\mathrm{R}_{x}(n)+1$ для всех $n$.

Пусть слово $v$ длины $n$ входит в $M(x)$, и $v=M(x)[i, j]-$ одно из таких вхождений при $i \geqslant h(n)$.

Заметим, что вхождения символов, встречающихся в $x$ конечное количество раз, ограничены префиксом длины $\mathrm{R}_{x}(1)$, поэтому, начиная с позиции $\mathrm{R}_{x}(1)$, преобразователь $M$ действует на $x$ как обратимый. При этом $i \geqslant h(n) \geqslant \mathrm{R}_{x}(1)$. 
Докажем, что найдется вхождение $[r, s]$ слова $v$ в $M(x)$ при $i<r \leqslant i+h(n)$.

Итак, пусть $v=M(x)[i, j]$, и $u_{1}=x[i, j]$ - прообраз $v$ в $x$, к которому $M$ подходит, находясь в состоянии $q_{1}$. Если бы $u_{1}$ входило в $x$ конечное количество раз, то $u_{1}$ не могло бы входить в $x[t, \infty)$ для $t \geqslant \mathrm{R}_{x}(n)$ по определению регулятора. Но $i \geqslant \mathrm{R}_{x}(n)$, поэтому $u_{1}$ входит в $x$ бесконечно много раз. Тогда найдется вхождение $u_{1}=x\left[i_{2}, j_{2}\right]$ такое, что $i_{2}>i$, но $j_{2} \leqslant i+\mathrm{R}_{x}(n)$. Если $M$ подходит к $i_{2}$ в состоянии $q_{1}$, то $M(x)\left[i_{2}, j_{2}\right]=v$. Иначе $M$ подходит к $i_{2}$ в некотором состоянии $q_{2} \neq q_{1}$ (в этом случае $m \geqslant 2$ ).

Положим $u_{2}=x\left[i, j_{2}\right]$. Имеем $\left|u_{2}\right| \leqslant \mathrm{R}_{x}(n)+1$. Тогда, поскольку $i \geqslant h(n) \geqslant$ $\mathrm{R}_{x}\left(\mathrm{R}_{x}(n)+1\right)$ (при $m \geqslant 2$ ), $u_{2}$ входит в $x$, бесконечно много раз. Поэтому найдется вхождение $u_{2}=x\left[i_{3}, j_{3}\right]$ такое, что $i_{3}>i$, но $j_{3} \leqslant i+\mathrm{R}_{x}\left(\mathrm{R}_{x}(n)+1\right)$. Если $M$ подходит к $j_{3}-n+1$ в состоянии $q_{1}$, то $M(x)\left[j_{3}-j+1, j_{3}\right]=v$, так как $u_{2}$ заканчивается словом $u_{1}$. Если $M$ подходит к $j_{3}-n+1$ в состоянии $q_{2}$, то в силу обратимости на $x[i, \infty)$ преобразователь $M$ подходит к $i_{2}$ в состоянии $q_{1}$, и тогда $M(x)\left[i_{2}, i_{2}+n-1\right]=v$, так как $u_{2}$ начинается с $u_{1}$. В худшем случае $M$ подходит к $j_{3}-n+1$ в некотором состоянии $q_{3}$, таком что $q_{3} \neq q_{1}$ и $q_{3} \neq q_{2}$ (рис. 6).

Рассуждая так дальше, найдем такие $j_{2}, \ldots, j_{m+1}$, что хотя бы к каким-то двум из позиций $i=j-n+1, j_{2}-n+1, \ldots, j_{m+1}-n+1$ преобразователь подходит в одинаковых состояниях. При этом $j_{m+1} \leqslant i+h(n)$.

Итак, мы доказали, что если слово длины $n$ входит в $M(x)[h(n), \infty)$, то оно входит в $M(x)$ бесконечно много раз. По лемме 2.2 .3 мы знаем тогда, что это слово входит в каждый отрезок длины $h(n)$ в $M(x)$. Отсюда $M(x) \in \mathscr{G} \mathscr{A} \mathscr{P}$ и $\mathrm{R}_{x}(n) \leqslant h(n)$. Предложение доказано.

Рассмотрев теперь разбиение $\mathrm{s}_{a}(x)$ последовательности $x$ для некоторой буквы $a$, входящей в $x$ бесконечно много раз, можно провести индукцию по количеству состояний преобразователя и, таким образом, другим способом доказать утверждение 1) теоремы 2.2.2. Как уже было сказано, в удобной форме оценку на регулятор образа, аналогичную той, что получена в утверждении 2) теоремы 2.2.2, нам получить не удалось, хотя с помощью описанного только что подхода, видимо, такую оценку получить можно, по крайней мере для некоторых частных случаев обобщенно почти периодических последовательностей.

ПроБлЕмА 4. Можно ли улучшить оценку на регулятор образа, полученную в утверждении 2) теоремы 2.2.2? Можно ли это сделать хотя бы в частных случаях, например, для заключительно почти периодических последовательностей? Для последовательностей с регулятором $\mathrm{R}_{x}(n)$, растущим достаточно быстро с ростом $n$ ? См. также проблему 3.

Нижние оценки мы обсуждаем в п. 2.5.

2.3. Блочное произведение. Пусть $u, v \in \mathbb{B}^{*}$. Определим блочное произведение $u \otimes v$ индукцией по длине $v$ :

$$
\begin{aligned}
u \otimes \Lambda & =\Lambda, \\
u \otimes v 0 & =(u \otimes v) u, \\
u \otimes v 1 & =(u \otimes v) \bar{u} .
\end{aligned}
$$


Легко проверить, что блочное произведение дистрибутивно справа (но не слева!) и ассоциативно по отношению к обычному приписыванию слов, т. е. $u \otimes$ $(v w)=(u \otimes v)(u \otimes w)$ и $u \otimes(v \otimes w)=(u \otimes v) \otimes w$ для любых слов $u, v, w$. Пусть теперь $u_{k}, k=0,1,2, \ldots,-$ последовательность непустых слов из $\mathbb{B}^{*}$ таких, что при $k \geqslant 1$ слово $u_{k}$ начинается с 0 . Тогда в последовательности $u_{0}, u_{0} \otimes u_{1}$, $u_{0} \otimes u_{1} \otimes u_{2}, \ldots$ каждое из слов является префиксом любого из следующих, и значит, существует

$$
\lim _{n \rightarrow \infty} \bigotimes_{k=0}^{n} u_{k}=\bigotimes_{k=0}^{\infty} u_{k}
$$

- бесконечная последовательность в $\mathbb{B}^{\mathbb{N}}$. Например, последовательность ТуэМорса - это блочное произведение слов $u_{k}=01$.

Блочное произведение, по-видимому, было введено в [52] и подробно изучалось в [45]. В частности, там доказано, что $\bigotimes_{k=0}^{\infty} u_{k}$ всегда почти периодична (см. теорему 2.4.1). Там же получен критерий периодичности: последовательность $x=\bigotimes_{k=0}^{n} u_{k}$ периодична тогда и только тогда, когда либо для некоторого $n$ выполнено $u_{k}=010101 \ldots 010$ для всех $k>n$, либо для некоторого $n$ выполнено $u_{k}=000 \ldots 00$ для всех $k>n$.

Кроме последовательности Туэ-Морса в качестве примера последовательности, получающейся при помощи блочного произведения, в [45] приводится последовательность

$$
x_{K}=0010011100010011101101100 \ldots=001 \otimes 001 \otimes 001 \otimes \cdots,
$$

названная там тернарной последовательностъю Кини или "вальсом бесконечного порядка". Также вводится частный случай блочного произведения - последовательности вида $0 p_{1} \otimes 0 p_{2} \otimes 0 p_{3} \otimes \cdots$, где все $p_{i}$ равны 0 или 1 , причем среди них бесконечное количество 1 ; они называются последовательностями Какутани, который их впервые изучал в [53]. Легко видеть, что множество последовательностей Какутани - это континуальное семейство почти периодических последовательностей; однако не всякая почти периодическая последовательность является последовательностью Какутани.

Блочное произведение - эффективный способ построения различных последовательностей, обладающих нужными свойствами. Примеры таких конструкций содержатся в [45], приведем некоторые результаты оттуда.

Относительной частотой вхождения $T_{u}(x, i, j)$ слова $u$ в последовательность $x$ на отрезке $[i, j]$ называется количество его вхождений на этом отрезке, деленное на длину отрезка. Блок $u$ называется чезаровским для $x$, если существует средняя частота его вхождения $I_{u}(x)=\lim _{n \rightarrow \infty} T_{u}(x, 0, n) ;$ равномерно чезаровским, если

$$
I_{u}(x)=\lim _{n \rightarrow \infty} T_{u}(x, s, s+n)
$$

равномерно по s. Последовательность (равномерно) чезаровская, если все ее факторы (равномерно) чезаровские.

Если $x$ почти периодична, то для любого ее фактора $u$ имеем строгое неравенство $\liminf \inf _{n \rightarrow \infty} \inf _{s \geqslant 0} T_{u}(x, s, s+n)>0$. В частности, если $u$ чезаровский, то $I_{u}(x)>0$. 
В [45] для последовательностей в алфавите $\{0,1\}$, получаемых с помощью блочного произведения, строится пример, в котором 0 и 1 не являются чезаровскими. Приводится критерий того, когда для таких последовательностей 0 является чезаровским со средней частотой $1 / 2$.

Кроме того, что особенно интересно для наших рассмотрений, в [45] обсуждается, насколько последовательности, получаемые при помощи блочного произведения, можно считать периодическими: "Хотя почти все наши последовательности не периодичны в строгом смысле, многие из них обладают свойствами периодичности несколько иного характера". Пример: для последовательности Туэ-Морса $\mathbf{t}$ и множества $F=\{0110,1001,0101,1010\}$ выполнено $\mathbf{t}[i, j] \in F \Leftrightarrow 2 \mid i$. Аналогично, для последовательности Кини и $F^{\prime}=\{001,110\}$ выполнено $x_{K}[i, j] \in F^{\prime} \Leftrightarrow 3 \mid i$. Доказана следующая общая теорема.

Teорема 2.3.1 (см. [45]). 1) Пусть $x=0 P_{1} \otimes 0 P_{2} \otimes \cdots u C_{n}=0 P_{1} \otimes 0 P_{2} \otimes$ $\cdots \otimes 0 P_{n}$ для некоторого $n$. Если существует $j$ такое, что в $D=0 P_{n+1} \otimes$ $0 P_{n+2} \otimes \cdots \otimes 0 P_{n+j}$ входит одно из слов 001, 110, 011, 100, то для множества $F=\bigcup_{|u|=2|D|} C_{n} \otimes u$ выполняется $x[i, j] \in F \Leftrightarrow\left|C_{n}\right| \mid i$.

2) Если последовательность $x=0 P_{1} \otimes 0 P_{2} \otimes \cdots$ не периодична, то для каждого $n$ найдется множество $F_{n}$, для которого $x[i, j] \in F_{n} \Leftrightarrow r_{n} \mid i$, где $C_{n}=0 P_{1} \otimes 0 P_{2} \otimes \cdots \otimes 0 P_{n}, r_{n}=\left|C_{n}\right|$.

В конце работы [45] сформулированы 8 задач, есть как простые упражнения, так и исследовательские проблемы, предложения обобщить полученные в статье результаты. Одна из задач заключается в предложении обобщить блочное произведение на случай произвольного конечного алфавита. Это сделано явно в [54], однако фактически все нужные конструкции содержатся в [11] (см. также п. 2.4).

Приведем следующий любопытный пример. Он также понадобится в доказательстве предложения 2.3.3.

ПРЕДЛОЖЕНИЕ 2.3.2. Существует почти периодическая последовательность над $\mathbb{B}$, у которой для любого $n$ найдется префикс, в котором нулей на $n$ больше, чем единии, и для любого $n$ найдется префикс, в котором единии, на п больше, чем нулей.

ДокАЗАТЕЛЬство. Положим $x=001 \otimes \bigotimes_{i=1}^{\infty} 0111$. Пусть $u_{m}=001 \otimes$ $\bigotimes_{i=1}^{m}$ 0111. Докажем индукцией по $m$, что $\left|u_{m}\right|_{0}-\left|u_{m}\right|_{1}=(-1)^{m} 2^{m}$. Действительно, при $m=0$ имеем $u_{0}=001,\left|u_{0}\right|_{0}-\left|u_{1}\right|_{1}=2-1=1$.

Пусть теперь $\left|u_{m}\right|_{0}-\left|u_{m}\right|_{1}=(-1)^{m} 2^{m}$. По определению $u_{m+1}=u_{m} \bar{u}_{m} \bar{u}_{m} \bar{u}_{m}$, значит,

$$
\begin{aligned}
\left|u_{m+1}\right|_{0}-\left|u_{m+1}\right|_{1} & =\left|u_{m}\right|_{0}+3\left|u_{m}\right|_{1}-\left(\left|u_{m}\right|_{1}+3\left|u_{m}\right|_{0}\right) \\
& =2\left(\left|u_{m}\right|_{1}-\left|u_{m}\right|_{0}\right)=(-1)^{m+1} 2^{m+1} .
\end{aligned}
$$

Предложение доказано.

В п. 2.2 обсуждались вопросы сохраняемости относительно конечно-автоматных преобразований различных свойств типа почти периодичности. Интересно понять, что произойдет, если мы несколько расширим класс рассматриваемых преобразований. 
Естественным обобщением можно считать счетчиковый конечно-автоматный преобразователь. Счетчиковый конечно-автоматный преобразователь неформально можно описать как конечно-автоматный преобразователь со счетчиком - переменной, в которой хранится натуральное число. На каждом шаге преобразователь может увеличить значение счетчика на 1, уменьшить на 1 или оставить без изменений. Преобразователь видит, равно ли значение счетчика нулю (но не видит значение счетчика), и по этой информации, а также по текущему состоянию и входному символу определяет, что делать со счетчиком и что выдавать на выход. Преобразователь не может уменьшать значение счетчика, если оно нулевое.

Как и следовало ожидать, счетчиковые конечно-автоматные преобразователи не сохраняют свойство обобщенной почти периодичности.

ПрЕДЛОЖЕНИЕ 2.3.3 [12]. Существуют счетчиковый конечно-автоматный преобразователь и обобщенно почти периодическая последовательность, которая под действием этого преобразователя переходит в последовательность, не являющуюся обобщенно почти периодической.

ДокАЗАТЕЛЬСТво. По предложению 2.3.2 существует почти периодическая последовательность в алфавите $\mathbb{B}$, обладающая следующим свойством: для любого $n \in \mathbb{N}$ найдется такой ее префикс, в котором нулей на $n$ больше, чем единиц, а также найдется такой префикс, в котором единиц на $n$ больше, чем нулей. Применим к этой последовательности следующий счетчиковый конечно-автоматный преобразователь. У него два режима, $a$ (начальный) и $b$. В режиме $a$ он действует так: увидев в последовательности символ 0, он увеличивает значение счетчика на один, а увидев 1, уменьшает значение счетчика на один. Когда счетчик обнуляется (что соответствует начальному отрезку последовательности с равным количеством 0 и 1), преобразователь переключается в режим $b$. В режиме $b$ преобразователь действует противоположным образом: при входе 1 увеличивает значение счетчика на один, при входе 0 уменьшает значение счетчика на один, при обнулении счетчика переключается в режим $a$. В выходную последовательность преобразователь подает всегда название своего режима. Видно тогда, что в выходной последовательности найдутся сколь угодно длинные отрезки подряд идущих $a$, и значит, она не является обобщенно почти периодической. Предложение доказано.

Счетчиковые конечно-автоматные преобразователи являются частным случаем конечно-автоматных преобразователей с магазинной памятью (стековых).

\section{4. Универсальный метод построения обобщенно почти периоди-} ческих последовательностей. Следующий метод построения последовательностей, по существу предложенный в [11] и обсуждавшийся также в [46], в некотором смысле обобщает блочное произведение. Он является универсальным, в том смысле, что с его помощью можно получить любые обобщенно почти периодические последовательности. Мы изложим немного модифицированную версию этого метода. Этот метод нам неоднократно понадобится далее в настоящем обзоре.

Последовательность $\left\langle B_{n}, C_{n}, l_{n}\right\rangle$, где $B_{n}$ и $C_{n}$ - непустые множества непустых слов в фиксированном конечном алфавите $A, l_{n}$ - натуральные числа, 
называется $\mathscr{G} \mathscr{A} \mathscr{P}$-схемой, если для нее выполняются следующие четыре условия для любого $n \in \mathbb{N}$ :

1) все слова из $B_{n}$ имеют длину $l_{n}$;

2) все слова из $C_{n}$ представимы в виде $v_{1} v_{2}$, где $v_{1}, v_{2} \in B_{n}$, и каждое слово из $B_{n}$ используется в качестве $v_{i}$ в каком-то из слов множества $C_{n}$;

$3)$ каждое слово из $B_{n+1}$ имеет вид $v_{1} v_{2} \ldots v_{k}$, где $v_{i} \in B_{n}$ для каждого $i \leqslant k$ и $v_{i} v_{i+1} \in C_{n}$ для каждого $i<k$, и для каждого $w \in C_{n}$ найдется $i<k$, для которого $w=v_{i} v_{i+1}$;

4) для каждого слова $u=v_{1} v_{2} \ldots v_{k} w_{1} w_{2} \ldots w_{k}$ из $C_{n+1}$ (здесь $v_{i}, w_{i} \in B_{n}$ ) имеем $v_{k} w_{1} \in C_{n}$.

Мы будем говорить, что последовательность $x \in A^{\mathbb{N}} \mathscr{G} \mathscr{A} \mathscr{P}$-порождена $\mathscr{G} \mathscr{A} \mathscr{P}$-схемой $\left\langle B_{n}, C_{n}, l_{n}\right\rangle$, если для каждого $n \in \mathbb{N}$ найдется такое $k_{n}$, что для всех $i \in \mathbb{N}$ выполнено

$$
x\left[k_{n}+i l_{n}, k_{n}+(i+2) l_{n}-1\right] \in C_{n} .
$$

Будем говорить, что последовательность $\mathscr{G} \mathscr{A} \mathscr{P}$-порождена правильно, если $l_{n} \mid k_{n}$.

Несложно видеть (из компактности), что каждая $\mathscr{G} \mathscr{A} \mathscr{P}$-схема правильно $\mathscr{G} \mathscr{A} \mathscr{P}$-порождает хотя бы одну последовательность. Как доказано в [46], каждая последовательность, $\mathscr{G} \mathscr{A} \mathscr{P}$-порожденная $\mathscr{G} \mathscr{A} \mathscr{P}$-схемой, является обобщенно почти периодической. Кроме того, каждая обобщенно почти периодическая последовательность $\mathscr{G} \mathscr{A} \mathscr{P}$-порождается некоторой $\mathscr{G} \mathscr{A} \mathscr{P}$-схемой, причем эту $\mathscr{G} \mathscr{A} \mathscr{P}$-схему можно выбрать так, чтобы последовательность порождалась правильно. Отметим также важное свойство эффективности: если обобщенно почти периодическая последовательность $\mathscr{G} \mathscr{A} \mathscr{P}$-порождена $\mathscr{G} \mathscr{A} \mathscr{P}$-схемой, то, зная последовательности $k_{n}$ и $l_{n}$, можно вычислять оценку сверху на регулятор почти периодичности порождаемой последовательности (здесь видно, что, возможно, логично было бы добавить последовательность $k_{n}$ в определение схемы - мы не сделали это из соображений краткости).

Для получения почти периодических последовательностей можно также пользоваться понятием $\mathscr{G} \mathscr{A} \mathscr{P}$-схемы. Мы будем говорить, что последовательность $x \in A^{\mathbb{N}} \mathscr{A} \mathscr{P}$-порождена $\mathscr{G} \mathscr{A} \mathscr{P}$-схемой $\left\langle B_{n}, C_{n}, l_{n}\right\rangle$, если для всех $n \in \mathbb{N}$ и $i \in \mathbb{N}$ выполнено

$$
x\left[i l_{n},(i+2) l_{n}-1\right] \in C_{n} .
$$

Другими словами, последовательность $\mathscr{A} \mathscr{P}$-порождается, если она $\mathscr{G} \mathscr{A} \mathscr{P}$-порождается со всеми $k_{n}=0$.

Аналогично предыдущему, каждая $\mathscr{G} \mathscr{A} \mathscr{P}$-схема $\mathscr{A} \mathscr{P}$-порождает хотя бы одну последовательность. Каждая последовательность, $\mathscr{A} \mathscr{P}$-порожденная $\mathscr{G} \mathscr{A} \mathscr{P}$-схемой, является почти периодической. Каждая почти периодическая последовательность может быть $\mathscr{A} \mathscr{P}$-порождена какой-нибудь $\mathscr{G} \mathscr{A} \mathscr{P}$-схемой. (Доказательства всех этих фактов аналогичны доказательствам упомянутых выше фактов из [46].) Свойство эффективности сохраняется: по последовательности $l_{n}$ в $\mathscr{G} \mathscr{A} \mathscr{P}$-схеме, порождающей последовательность, можно получить оценку сверху на регулятор почти периодичности этой последовательности.

Этот способ порождения почти периодических последовательностей можно упростить, но пожертвовав, по всей видимости, свойством эффективности. 
Последовательность $\left\langle B_{n}, l_{n}\right\rangle$, где $B_{n}$ - непустое множество непустых слов в фиксированном конечном алфавите $A, l_{n}$ - натуральные числа, называется $\mathscr{A} \mathscr{P}$-схемой, если для нее для любого $n \in \mathbb{N}$ выполнено условие 1$)$, и для любого $n \in \mathbb{N}$ каждое $u \in B_{n+1}$ имеет вид $u=v_{1} v_{2} \ldots v_{k}$, где $v_{i} \in B_{n}$, причем для каждого $w \in B_{n}$ найдется $i$ такое, что $v_{i}=w$. Последовательность $x$ $\mathscr{A} \mathscr{P}$-порождена $\mathscr{A} \mathscr{P}$-схемой, если для любых $i$ и $n$

$$
x\left[i l_{n},(i+1) l_{n}-1\right] \in B_{n} .
$$

Как и раньше, каждая $\mathscr{A} \mathscr{P}$-схема $\mathscr{A} \mathscr{P}$-порождает хотя бы одну последовательность. Каждая последовательность, $\mathscr{A} \mathscr{P}$-порожденная $\mathscr{A} \mathscr{P}$-схемой, является почти периодической. Каждая почти периодическая последовательность может быть $\mathscr{A} \mathscr{P}$-порождена какой-нибудь $\mathscr{A} \mathscr{P}$-схемой. (Опять же, доказательства этих фактов аналогичны доказательствам упомянутых выше фактов из [46].) Однако теперь уже, вообще говоря, может быть неверно, что по схеме можно оценивать сверху регулятор почти периодичности порождаемой последовательности. По крайней мере, то же рассуждение, которое можно было применить раньше, здесь уже не работает. Например, неясно даже, как, зная схему, дать оценку на расстояния между соседними вхождениями двухбуквенных слов: такое слово может оказаться на стыке двух слов - элементов схемы. Неизвестно, когда такой стык появится и появится ли вообще.

ГиПотезА 5. Существуют вычислимая $\mathscr{A} \mathscr{P}$-схема и $\mathscr{A} \mathscr{P}$-порожденная ею последовательность $x$ такая, что регулятор $\mathrm{R}_{x}$ не вычислим.

Когда ясно, о каком типе схемы или о каком типе порождения последовательности идет речь, мы будем опускать соответствующие приставки.

Применение универсального метода построения проиллюстрируем на примере следующего результата из [45], который мы теперь можем доказать совсем просто.

Tеорема 2.4.1 [45]. Пусть $x=\bigotimes_{k=0}^{\infty} u_{k}$, где слова $u_{k} \in \mathbb{B}^{*}$ начинаются с 0 при $k \geqslant 1$. Тогда $x$ почти периодическая.

ДокАЗАТЕЛЬство. Если все $u_{k}$, начиная с некоторого, состоят из одних 0 , то последовательность $x$ периодическая, а значит, и почти периодическая. Иначе достаточно рассмотреть случай, при котором каждое $u_{k}$ содержит хотя бы один символ 1 (общий случай к нему легко сводится). Но в этом случае $x$ $\mathscr{A} \mathscr{P}$-порождается $\mathscr{A} \mathscr{P}$-схемой $B_{n}=\left\{a_{n}, \bar{a}_{n}\right\}$, где $a_{n}=\bigotimes_{k=0}^{n} u_{k}$, и, значит, является почти периодической.

\section{5. Произведение последовательностей и почти периодичность}

при проекции. Результаты этого пункта дают любопытные примеры почти периодических последовательностей, подтверждающие, что свойства почти периодических последовательностей могут быть очень разнообразными.

На последовательностях можно определить операцию $\times$, которую мы будем называть (декартовым) произведением. Для $x \in A^{\mathbb{N}}, y \in B^{\mathbb{N}}$ определим $x \times y \in$ $(A \times B)^{\mathbb{N}}$ так, что

$$
(x \times y)(i)=\langle x(i), y(i)\rangle \text {. }
$$


Аналогично можно определить произведение произвольного количества последовательностей.

Как несложно видеть, если последовательность $y$ периодическая с периодом $m$, то $x \times y$ можно получить как результат равномерного конечно-автоматного преобразования последовательности $x$ преобразователем с $m$ состояниями. Такой преобразователь мы будем называть ииклическим.

Отсюда получаем такое следствие из предложения 2.2.6.

СлеДСтвие 2.5.1. Если $x \in \mathscr{A} \mathscr{P} u y \in \mathscr{P}$, mo $x \times y \in \mathscr{A} \mathscr{P}$.

Результат следствия 2.5.1 был впервые получен в [55].

Следующий результат (теорема 2.5.2) можно считать нижней оценкой для утверждения 2) теоремы 2.2.2. В утверждении 2) теоремы 2.2.2 дается верхняя оценка на регулятор почти периодичности образа обобщенно почти периодической последовательности при конечно-автоматном преобразовании. Если исходная последовательность $x$ имеет регулятор $\mathrm{R}_{x}$, и автомат имеет $m$ состояний, то регулятор образа можно оценить сверху величиной порядка $\mathrm{R}_{x}^{2 m}$ (где верхний индекс $m$, как и в п. 2.2, понимается в смысле композиции функций, а не в смысле возведения в степень значения функции). Теорема 2.5.2 показывает, что даже для простейшего типа конечно-автоматных преобразований, осуществляемых циклическими преобразователями, эту оценку на регулятор образа нельзя существенно улучшить.

Обозначим через $C_{m}$ последовательность $01 \ldots(m-1) 01 \ldots(m-1) 01 \ldots$ в алфавите из $m$ символов $0,1, \ldots, m-1$. Мы пишем $f \geqslant g$ для функций $f, g$, если $f(x) \geqslant g(x)$ для всякого аргумента $x$.

Tеорема 2.5.2 [13], [56]. Для каждого $m \geqslant 12$ существует бесконечно много последовательностей $x \in \mathscr{A} \mathscr{P}$ таких, что $\mathrm{R}_{x \times C_{m}} \geqslant \mathrm{R}_{x}^{\lfloor m / 4\rfloor-3}$.

Как сказано выше, по-другому теорему 2.5.2 можно сформулировать как нижнюю оценку на регулятор почти периодичности образа последовательности $x$ под действием циклического конечно-автоматного преобразователя. С помощью простой модификации этого преобразователя можно в качестве следствия из теоремы 2.5.2 получить нижнюю оценку вида $\operatorname{pr}\left(x \times C_{m}\right) \geqslant \mathrm{R}_{x}^{\alpha n-\beta}(1)$, для некоторых положительных действительных $\alpha, \beta$, дополняющую теорему 2.2.5, в которой дается соответствующая верхняя оценка. Подробнее см. [56].

В качестве естественного продолжения этой темы, можно задаться следующим неформальным вопросом: что можно сказать о произведении $x \times y$ последовательностей $x, y \in \mathscr{A} \mathscr{P}$ ?

Tеорема 2.5.3 [57]. Для каждого из множеств $\mathcal{M}=\mathscr{E} \mathscr{A} \mathscr{P} \backslash \mathscr{A} \mathscr{P}, \mathscr{G} \mathscr{A} \mathscr{P} \backslash$ $\mathscr{E} \mathscr{A} \mathscr{P}, \mathbb{B}^{\mathbb{N}} \backslash \mathscr{G} \mathscr{A} \mathscr{P}$ можно построить последовательности $x, y \in \mathscr{A} \mathscr{P}$ в албавите $\mathbb{B}$, maкие что $x \times y \in \mathcal{M}$.

Естественно исследовать свойства произведения последовательностей из каких-нибудь более узких классов, чем класс всех почти периодических последовательностей. Примерами результатов такого типа являются следствие 2.5.1 и теорема 2.5.7.

Рассмотрим множество $\mathscr{P} \mathscr{P}$ последовательностей $x$, для которых верно следующее: произведение $x$ с любой почти периодической последовательностью 
почти периодично. Например, следствие 2.5.1 утверждает, что множеству $\mathscr{P} \mathscr{P}$ принадлежат все периодические последовательности. По теореме 2.5.3 $\mathscr{P} \mathscr{P} \subsetneq$ $\mathscr{A} \mathscr{P}$. В [58] (а также в дальнейших частных сообщениях от П. Салимова) доказана для разнообразных последовательностей их принадлежность множеству $\mathscr{P} \mathscr{P}$, а именно:

1) для широкого класса чисто морфических последовательностей, в частности, для последовательности Туэ-Морса;

2) для последовательностей Тёплица и их обобщений;

3) для "правильных" вращательных последовательностей (т. е. таких, для которых траектория точки никогда не попадает на границу между дугами разбиения).

Для всех полученных результатов интересно было бы получить оценку на регулятор почти периодичности произведения на основе регуляторов почти периодичности исходных последовательностей.

Подытожим интересные вопросы.

Проблема 6. 1) Пусть $x, y \in \mathscr{A} \mathscr{P}$. Существует ли какой-то критерий для определения, верно ли, что $x \times y \in \mathscr{G} \mathscr{A} \mathscr{P}$ ? Что $x \times y \in \mathscr{A} \mathscr{P}$ ?

2) При каких дополнительных условиях на $x$ и $y$ произведение $x \times y$ будет обладать некоторыми свойствами, близкими к периодичности?

3) Для тех случаев, когда в предыдущем пункте удается доказать почти периодичность произведения, выразить оценку на регулятор почти периодичности произведения через оценки на регуляторы сомножителей. Оценки такого типа могут позволить получить новые примеры последовательностей с разрешимыми логическими теориями (см. раздел 3 ).

В некотором смысле обобщением одного из утверждений теоремы 2.5.3 являются следующие три результата, доказанные явно в [46], но по существу полученные в [11]. В доказательствах существенным образом используется универсальный метод построения обобщенно почти периодических последовательностей (см. п. 2.4).

Tеорема 2.5.4 [11], [46]. Для любого $m \in \mathbb{N}$ существуют $m+1$ двоичных последовательностей таких, что произведение любых т из них является эффективно почти периодической последовательностью, а произведение всех $m+1$ последовательностей не явлется почти периодическим.

Теорема 2.5.5 [11], [46]. Для любого $m \in \mathbb{N}$ существуют $m+1$ двоичных последовательностей таких, что произведение любых т из них является эффективно почти периодической последовательностью, а произведение всех $m+1$ последовательностей является почти периодической последовательностью, но не является эффективно почти периодической.

Морфизм $h: A^{*} \rightarrow B^{*}$ назовем проекцией, если для любой буквы $a \in A$ выполнено $|h(a)|=1$, и при этом $|A|>|B|$.

Теорема 2.5.6 [11], [46]. Для любого $m \in \mathbb{N}$ существует вычислимая последовательность $x: \mathbb{N} \rightarrow\{1, \ldots, m\}$ такая, что для любой проекции $h$ последовательность $h(x)$ почти периодична с вычислимой верхней оценкой на 
регулятор почти периодичности. Последовательность х может быть построена удовлетворяющей одному из следующих условий:

1) $x$ не является почти периодической;

2) х является почти периодической последовательностью, но не является эффективно почти периодической.

В следующей теореме доказывается, что класс точно почти периодических последовательностей замкнут относительно операции умножения на периодическую последовательность.

Teоpema 2.5.7. Если $x \in \mathscr{P} \mathscr{A} \mathscr{P} u y \in \mathscr{P}$, mo $x \times y \in \mathscr{P} \mathscr{A} \mathscr{P}$.

ДоКАЗАТЕЛЬСтво. Будем считать, что $y=01 \ldots(m-1) 01 \ldots(m-1) 01 \ldots$, где $m$ - период $y$. Случай произвольного $y \in \mathscr{P}$ получается из этого кодированием, которое, очевидно, сохраняет $\mathscr{P} \mathscr{A} \mathscr{P}$. В этом доказательстве для краткости вместо $u \times[i, i+|u|-1]$ будем писать $u \times[i, \cdot]$.

Пусть $u$ входит в $x$ и

$$
A=\{i: 0 \leqslant i \leqslant m, u \times[j, \cdot] \text { входит в } z \text { для некоторого } j \equiv i(\bmod m)\} .
$$

Пусть $v \times[a, \cdot]$ - такое подслово $z$, которое для каждого $i \in A$ содержит вхождение $u \times[j, \cdot]$ для некоторого $j \equiv i(\bmod m)$. Слово $v$ входит в $x$, поэтому для некоторых $b, d$ имеем $x[b+d t, b+d t+|v|-1]=v$ для каждого $t \in \mathbb{N}$. Ясно при этом, что

$$
\begin{array}{r}
B=\{i: 0 \leqslant i \leqslant m, u \times[j, \cdot] \text { входит в } v \times[b, \cdot] \\
\text { для некоторого } j \equiv i(\bmod m)\}=A .
\end{array}
$$

Действительно, по определению $A$ имеем $B \subseteq A$, но $B=\{(i+b-a) \bmod m$ : $i \in A\}$. Значит, $\# B=\# A$, откуда $B=A$.

Таким образом, если $u \times[s, \cdot]$ входит в $z$, то для некоторого $p \equiv s(\bmod m)$ имеем вхождение $u \times[p, \cdot]$ в $v \times[b, \cdot]$. Поэтому $u \times[p+t d m, \cdot]$ входит в $z$ для каждого $t \in \mathbb{N}$. Следовательно, $z \in \mathscr{P} \mathscr{A} \mathscr{P}$. Теорема доказана.

В связи с теоремой 2.5.7 возникает следующий вопрос.

ПроБлЕмА 7. 1) Верно ли, что класс точно почти периодических последовательностей замкнут относительно конечно-автоматных преобразований?

2) Если ответ на предыдущий вопрос положительный, то можно ли это доказать в каком-нибудь смысле эффективно и получить более простой критерий разрешимости монадической теории точно почти периодической последовательности (см. раздел 3)?

\section{3. Логика почти периодических последовательностей}

3.1. Логические теории и автоматы. Рассмотрим следующие простые вопросы о последовательности $x$ : входит ли в $x$ символ $a$ ? Входит ли слово $u$ ? Входит ли слово $u$ бесконечно много раз? Можно сформулировать и более сложные вопросы про последовательность. Когда на такие вопросы можно отвечать алгоритмически, получая на вход последовательность? 
Различные, в том числе и перечисленные только что, свойства бесконечных последовательностей могут быть выражены в логической теории первого nорядка. Под такой теорией для последовательности $x \in A^{\mathbb{N}}$ мы будем понимать следующее. Формально, в качестве структуры возьмем $\langle\mathbb{N}, S,<, X\rangle$, где $\mathbb{N}$ - множество натуральных чисел, которое пробегают индивидные переменные, $S$ - двухместный предикат следования $(S(x, y)$ истинно, если и только если $y$ следует за $x$, т. е. $y=x+1),<-$ двухместный предикат порядка на натуральных числах, $X$ - функциональный символ, интерпретируемый как последовательность $x: \mathbb{N} \rightarrow A$. В качестве теории берем обычную теорию первого порядка, истинность формул интерпретируем естественным образом. Во всех рассматриваемых нами теориях мы подразумеваем наличие двухместного предиката равенства, интерпретируемого естественным образом.

Ясно, что у такой реализации есть много вариантов, эквивалентных между собой по выразительным способностям. Например, можно было вместо двухместного предиката следования взять в структуру одноместную функцию следования. Можно было и не брать предикат следования вообще, потому что он выразим через неравенство. Точно так же вместо одной функции $X$ можно было взять семейство предикатов $X_{a}$ по одному для каждого $a \in A$, истинных ровно там, где в $x$ стоит буква $a$. Можно обойтись и меньшим - логарифмическим по отношению к размеру алфавита последовательности - количеством предикатов. Кроме того, поскольку константа 0 выразима в определенной выше структуре, ясно, что можно было, не меняя множество выразимых формул, добавить в структуру все константы. Мы можем переходить от одной конкретной реализации к другой, если понадобится, при этом четко фиксируя, какая именно реализация теории рассматривается, когда это важно, как, например, в теореме 3.1.1.

Теорию, определенную выше, будем обозначать $\mathrm{T}\langle\mathbb{N},<, x\rangle$.

Несложно видеть, что простые свойства последовательности $x$, типа упомянутых в начале этого пункта, можно выразить в теории $\mathrm{T}\langle\mathbb{N},<, x\rangle$. Например, формула $\forall p \exists q \exists r q>p \wedge S(q, r) \wedge X(q)=0 \wedge X(r)=1$ означает свойство вхождения в $x$ бесконечное количество раз слова 01 . Тем не менее, некоторые просто формулируемые свойства, которые, возможно, хотелось бы выразить, в теории первого порядка выразить нельзя, например, “слово в алфавите $\{a, b\}$, в котором между любыми последовательными вхождениями $b$ (такими, между которыми нет других вхождений $b$ ) входит нечетное количество букв $a$ ".

Указанное свойство, однако, можно выразить в более сильной монадической теории (второго порядка). В такой теории, кроме обычных переменных по натуральным числам $p, q, \ldots$, разрешены также монадические переменные по множествам натуральных чисел (или по одноместным предикатам) $P, Q, \ldots$ Вводятся соответствующие атомарные формулы вида $P(p), Q(p), \ldots$, обозначающие " $p$ принадлежит $P$ ", " $p$ принадлежит $Q$ ", . . Р Разрешены также кванторы по монадическим переменным (наряду с кванторами по числам). Такую теорию мы будем обозначать $\mathrm{MT}\langle\mathbb{N},<, x\rangle$.

Теории, аналогичные вышеперечисленным, но не расширенные последовательностью, будем обозначать соответственно $\mathrm{T}\langle\mathbb{N},<\rangle, \mathrm{MT}\langle\mathbb{N},<\rangle$. 
Теория $\mathrm{MT}\langle\mathbb{N},<, x\rangle$ богаче теории $\mathrm{T}\langle\mathbb{N},<, x\rangle$. Например, упомянутое выше не выразимое в теории первого порядка свойство в монадической теории выражается так:

$$
\begin{aligned}
\forall p \forall q((X(p)=b) & \wedge(X(q)=b) \wedge(p<q) \wedge \forall r((p<r) \wedge(r<q) \rightarrow(X(r)=a)) \\
& \rightarrow \exists Y(\forall u \forall v(S(u, v) \rightarrow(Y(u) \leftrightarrow \neg Y(v))) \wedge Y(p) \wedge Y(q))) .
\end{aligned}
$$

Как и в случае с теориями первого порядка, в описанной формализации монадической теории многое можно реализовать по-другому. Например, можно отказаться от неравенства, потому что в монадической теории оно выразимо через следование. Как и в случае теорий первого порядка, мы можем переходить от одной реализации к другой для удобства.

Произвольное подмножество множества $\left(A^{\mathbb{N}}\right)^{n}$ (для произвольного $n$ ) будем называть языком.

Для формулы $\varphi\left(X_{1}, \ldots, X_{n}\right)$ в любом из вышеописанных языков (с $n$ свободными монадическими переменными $\left.X_{1}, \ldots, X_{n}\right)$ будем обозначать через $L(\varphi)$ множество всех $x=\left\langle x_{1}, \ldots, x_{n}\right\rangle \in\left(A^{\mathbb{N}}\right)^{n}$, для которых эта формула верна, если интерпретировать $X_{i}$ как $x_{i}$.

Естественным и основным для нас вопросом о любой теории является вопрос о ее разрешимости. Теория разрешима, если существует алгоритм, который по любой замкнутой формуле определяет ее истинность.

Следующий результат о теориях первого порядка был получен в [10].

Теорию $\mathrm{T}^{\prime}\langle\mathbb{N},<, x\rangle$ (незначительную модификацию теории $\mathrm{T}\langle\mathbb{N},<, x\rangle$ ) определим следующим образом. Пусть $P$ - система предикатов, задающая последовательность $x$. Термами теории будут выражения вида $c, x+c$, где $c \in \mathbb{Z}$, $x$ - переменная. Атомарными формулами теории будут выражения $\tau<\tau^{\prime}$, $\tau>\tau^{\prime}, p(\tau)$, где $p$ - предикат из системы $P, \tau, \tau^{\prime}$ - термы. Несмотря на то, что значения термов могут не лежать в $\mathbb{N}$, отношения, задаваемые атомарными формулами, естественным образом всюду определены на $\mathbb{N}$.

Мы говорим, что теория бескванторная, если для каждой формулы теории найдется эквивалентная ей бескванторная.

ТЕОРема 3.1 .1 (см. [10]). 1) Если теория $\mathrm{T}^{\prime}\langle\mathbb{N},<, x\rangle$ бескванторная, то $x \in$ $\mathscr{G} \mathscr{A} \mathscr{P}$.

2) Пусть $x \in \mathscr{G} \mathscr{A} \mathscr{P}$. Тогда теория $\mathrm{T}^{\prime}\langle\mathbb{N},<, x\rangle$ бескванторная, причем она разрешима, если и только если $x$ эфбективно обобщенно почти периодична.

Обратимся теперь к монадическим теориям. Оказывается, что, благодаря применению теории автоматов, для достаточно широких классов последовательностей можно получить критерий разрешимости их монадических теорий.

Назовем (недетерминированным) автоматом Бюхи совокупность $M=\langle A$, $Q, \tilde{q}, \Delta, F\rangle$, где $A$ - входной алфавит, $Q$ - множество состояний, $\tilde{q} \in Q$ - начальное состояние, $\Delta \subseteq Q \times A \times Q$ - множество переходов, $F \subseteq Q$ - множество заключительных состояний. Ходом автомата $M$ на последовательности $x=x(0) x(1) x(2) \ldots$ называется такая последовательность состояний $\rho=\rho(0) \rho(1) \rho(2) \ldots$, что $\rho(0)=\tilde{q}$ и $\langle\rho(i), x(i), \rho(i+1)\rangle \in \Delta$ для любого $i$. Мы 
говорим, что автомат $M$ принимает $x$, если существует хотя бы один ход $\rho$ автомата $M$ на $x$, для которого хотя бы одно состояние, встречающееся в $\rho$ бесконечное количество раз, входит в множество заключительных состояний $F$. Такой ход будем называть принимающим. Определяя детерминированный автомат Бюхи, множество переходов $\Delta \subseteq Q \times A \times Q$ можно заменить на функцию переходов $\delta: Q \times A \rightarrow Q$ (с естественными изменениями для определения хода автомата).

Есть немного другой вариант понятия автомата на последовательностях. Назовем (недетерминированным) автоматом Мюллера совокупность $M=\langle A$, $Q, \tilde{q}, \Delta, \mathscr{F}\rangle$, где $A$ - входной алфавит, $Q$ - множество состояний, $\tilde{q} \in Q$ - начальное состояние, $\Delta \subseteq Q \times A \times Q-$ множество переходов, $\mathscr{F} \subseteq 2^{Q}$ - множество заключительных макросостояний. Здесь под макросостоянием мы понимаем элемент множества $2^{Q}$, т. е. произвольное подмножество множества состояний $Q$. Ходом автомата $M$ на последовательности $x=x(0) x(1) x(2) \ldots$ называется такая последовательность состояний $\rho=\rho(0) \rho(1) \rho(2) \ldots$, что $\rho(0)=\tilde{q}$ и $(\rho(i), x(i), \rho(i+1)) \in \Delta$ для любого $i$. Назовем пределом (или предельным макросостоянием) автомата $M$ на последовательности $x$ вдоль хода $\rho$ множество всех таких состояний, которые встречаются в $\rho$ бесконечное количество раз, обозначим это множество через $\lim _{\rho} M$. Мы говорим, что автомат $M n p u-$ нимает $x$, если существует хотя бы один ход $\rho$ автомата $M$ на $x$, для которого $\lim _{\rho} M \in \mathscr{F}$. Такой ход будем называть принимающим. Другими словами, слово принимается, если хотя бы на каком-нибудь ходе предельное макросостояние принадлежит множеству заключительных макросостояний $\mathscr{F}$. Аналогично предыдущему, определяя детерминированный автомат Мюллера, множество переходов $\Delta \subseteq Q \times A \times Q$ можно заменить на функцию переходов $\delta: Q \times A \rightarrow Q$ (с естественными изменениями для определения хода автомата).

Автоматы Бюхи и Мюллера имеет смысл рассматривать и для кортежей последовательностей (которые являются последовательностями над бо́льшим алфавитом). Для автомата $M$ Бюхи или Мюллера на кортежах из $n$ последовательностей над $A$ множество всех $x=\left\langle x_{1}, \ldots, x_{n}\right\rangle \in\left(A^{\mathbb{N}}\right)^{n}$, которые принимаются автоматом $M$, обозначим $L(M)$.

Для примера рассмотрим множество $L$ последовательностей в алфавите $\{a, b, c\}$, в которые если $a$ входит бесконечное количество раз, то и $b$ входит бесконечное количество раз. Автоматы Мюллера и Бюхи, принимающие в точности слова множества $L$, показаны на рис. 7 (автомат Бюхи понадобился недетерминированный).

Это пример общей ситуации.

ТЕОРема 3.1.2 [59]. Недетерминированные автоматы Бюхи, недетерминированные автоматы Мюллера и детерминированные автоматы Мюллера распознают один и тот же класс языков. Более того, по автомату одного типа можно получать эквивалентный автомат другого типа алгоритмически.

Язык, распознаваемый автоматом Мюллера или автоматом Бюхи, будем называть регулярным. Детерминированные автоматы Бюхи распознают меньший класс языков. 

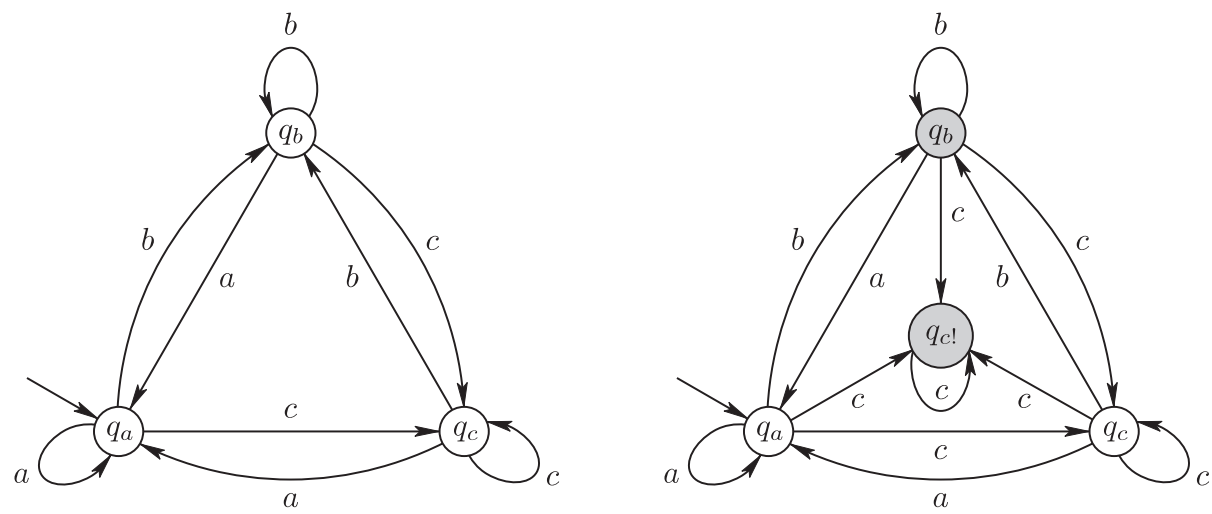

$\varnothing,\left\{q_{b}\right\},\left\{q_{c}\right\},\left\{q_{a}, q_{b}\right\},\left\{q_{b}, q_{c}\right\},\left\{q_{a}, q_{b}, q_{c}\right\}$

Рис. 7. Пример автомата Мюллера (слева) и автомата Бюхи (справа), принимающих одно и то же множество последовательностей. Множество принимающих макросостояний автомата Мюллера дано списком, заключительные состояния автомата Бюхи заштрихованы

Оказывается, что между описанием языков с помощью конечных автоматов и описанием их формулами монадического языка есть прямая связь.

ТЕОРема 3.1 .3 [60]. Существует алгоритм, который по каждому автомату Бюхи $M$ строит формулу ч монадической теории, такую что $L(M)=$ $L(\varphi)$, и наоборот, по любой бормуле $\varphi$ строит такой автомат Бюхи $M$, что $L(\varphi)=L(M)$. Теория $\mathrm{MT}\langle\mathbb{N},<\rangle$ разрешима.

Нас же интересует ситуация, когда теория $\mathrm{MT}\langle\mathbb{N},<\rangle$ расширена последовательностью. Оказывается, и в этом случае теорема Бюхи (теорема 3.1.3) применима. Несложно видеть, что выполняется следующее следствие.

СледствиЕ 3.1.4. Монадическая теория последовательности х разрешима тогда и только тогда, когда существует алгоритм, который по любому автомату Бюхи (или любому детерминированному автомату Мюллера) может определить, принимает ли этот автомат последовательность $x$ или нет.

Следующий основной результат является важнейшим примером применения результатов п. 2.2, в частности, теоремы 2.2.2.

ТЕОРема 3.1 .5 [11]. Теория $\mathrm{MT}\langle\mathbb{N},<, x\rangle$ обобщенно почти периодической последовательности х разрешима тогда и только тогда, когда х эффективно обобщенно почти периодична.

Доказательство. $\Rightarrow$. Пусть для $x \in \mathscr{G} \mathscr{A} \mathscr{P}$ теория $\mathrm{MT}\langle\mathbb{N},<, x\rangle$ разрешима. Тогда для каждого $n$ и для каждого возможного символа $a$ проверяем, не верно ли $x(n)=a$, и так перебором находим значение $x(n)$. Таким образом, $x$ вычислима. Также перебором можно вычислять $\mathrm{R}_{x}$, поскольку для любых $n$ и $l$ можно записать формулой тот факт, что $\mathrm{R}_{x}(n) \leqslant l$. 
$\Leftarrow$. Пусть теперь $x \in \mathscr{G} \mathscr{A} \mathscr{P}$ вычислима и некоторая $f \geqslant \mathrm{R}_{x}$ вычислима.

По следствию 3.1.4 для разрешимости $\mathrm{MT}\langle\mathbb{N},<, x\rangle$ достаточно уметь по любому детерминированному автомату Мюллера, запущенному на $x$, определять, принимает ли он $x$ или нет.

Пусть $M$ - какой-нибудь детерминированный автомат Мюллера, действующий на $x$. Рассмотрим конечно-автоматный преобразователь $M^{\prime}$, полученный из $M$ следующим образом: внутреннее устройство $M^{\prime}$ такое же, как и у $M$, при этом про принимающие макросостояния $M$ мы забываем. На входных последовательностях $M^{\prime}$ работает точно так же, как и $M$, а на выход выдает на каждом шаге свое текущее состояние. По теореме 2.2 .2 , п. 3) из вычислимости $x$ и $f$ следует вычислимость $F(x) \in \mathscr{G} \mathscr{A} \mathscr{P}$ и некоторой $g \geqslant \mathrm{R}_{M^{\prime}(x)}$.

Легко вычислить, какие символы входят в $M^{\prime}(x)$ бесконечно много раз: для этого достаточно посмотреть, какие символы входят в отрезок $M^{\prime}(x)[g(1)$, $2 g(1)-1]$ (а "посмотреть" мы можем, потому что сама последовательность $M^{\prime}(x)$ тоже вычислима).

Таким образом, мы можем найти множество всех таких состояний автомата $M$, которые встречаются бесконечное количество раз в процессе работы $M$ на $x$. Значит, мы можем проверить, принимает ли автомат $M$ последовательность $x$. Теорема доказана.

СлеДСтвиЕ 3.1.6. Теория $\mathrm{MT}\langle\mathbb{N},<, x\rangle$ почти периодической последовательности $x$ разрешима тогда и только тогда, когда $x$ вычислима и множество подслов $\operatorname{Fac}(x)$ разрешимо.

ДокАЗАТЕЛЬСтво. Следует из теоремы 3.1.5 и предложения 1.5.2.

Проблема 8. Предположим, последовательность $x \in \mathscr{E} \mathscr{A} \mathscr{P}(\mathscr{G} \mathscr{A} \mathscr{P}, \mathscr{R}, \mathscr{E} \mathscr{R})$ вычислима. Верно ли, что теория $\mathrm{MT}\langle\mathbb{N},<, x\rangle$ разрешима, если множество подслов $\mathrm{Fac}(x)$ разрешимо? Каков ответ, если дополнительно известно, что можно вычислить про каждое слово, входит ли оно в $x$ бесконечно много раз? Для всех этих вопросов есть и различные равномерные варианты. Например, существует ли алгоритм, который по последовательности $x \in \mathscr{E} \mathscr{A} \mathscr{P}$, числу $l \geqslant \operatorname{pr}(x)$ и множеству подслов последовательности $x[l, \infty) \in \mathscr{A} \mathscr{P}$ разрешает теорию $\mathrm{MT}\langle\mathbb{N},<, x\rangle$ ? В этой связи см. также результаты раздела 4.

По-видимому, ответы на все эти вопросы отрицательны, хотя степень уверенности авторов в этом различна для разных из них.

3.2. Разрешимость теорий конкретных последовательностей. Покажем, как полученные в п. 3.1 критерии применить к некоторым ранее рассмотренным примерам последовательностей.

Теорема 3.2.1. Монадическая теория $\mathrm{MT}\langle\mathbb{T},<, \mathbf{t}\rangle$ последовательности Туэ-Морса разрешима.

ДокАЗАтЕльство. Напомним, что последовательность Туэ-Морса можно определить как $\mathbf{t}=\lim _{n \rightarrow \infty} u_{n}$, где последовательность слов $u_{n}$ определяется по индукции: $u_{0}=0$ и $u_{n+1}=u_{n} \bar{u}_{n}$. При этом $\left|u_{n}\right|=2^{n}$. (См. п. 1.1.)

Ясно, что t вычислима. По следствию 3.1.6 достаточно доказать теперь, что $\operatorname{Fac}(\mathbf{t})$ разрешимо. Пусть $w$ - произвольное слово. Выберем $n$ так, чтобы выполнялось неравенство $|w| \leqslant 2^{n}$. Поскольку для любого $n$ имеем $\mathbf{t}=$ 
$u_{n} \bar{u}_{n} \bar{u}_{n} u_{n} \bar{u}_{n} u_{n} u_{n} \bar{u}_{n} \ldots$, слово $w$ входит в $\mathbf{t}$ тогда и только тогда, когда оно входит в одно из слов $u_{n} u_{n}, u_{n} \bar{u}_{n}, \bar{u}_{n} u_{n}, \bar{u}_{n} \bar{u}_{n}$, что легко проверяется. Теорема доказана.

Аналогично явно можно доказать разрешимость монадической теории последовательности Фибоначчи $\mathbf{f}$, но этот результат следует и из теоремы 3.2 .2 ниже.

Поставленный в [61] вопрос о разрешимости монадической теории последовательности $x_{n}=\lceil\sin (n)\rceil$ изначально послужил мотивацией в работах [10], [11]. Несложно видеть, что последовательность $x_{n}$ является вращательной. Частный случай вращательных последовательностей - последовательности Штурма. Для специальных последовательностей Штурма, т.е. таких, что сдвиг $\rho=0$, критерий эффективной почти периодичности (дающий в соответствии с теоремой 3.1.5 критерий разрешимости монадической теории) был получен в [10]. Критерий разрешимости для произвольных последовательностей Штурма (и для произвольных вращательных) следует из варианта теоремы 1.6.3 (сформулированного не в терминах алгебраичности, а в терминах вычислимости). Здесь мы доказываем его в явном виде (доказательство не сложнее предложенного в [10]). В [62] вопрос получения такого критерия также сформулирован.

Напомним, что действительное число $а$ называется вычислимым, если существует алгоритм, который по любому положительному рациональному числу $\varepsilon$ выдает приближение к $a$ с точностью $\varepsilon$, т. е. рациональное число в интервале $(a-\varepsilon, a+\varepsilon)$.

ТЕОрема 3.2.2. Монадическая теория $\mathrm{MT}\left\langle\mathbb{T},<, s_{\alpha, \rho}\right\rangle$ механической последовательности (т.е. последовательности Штурма) с наклоном $\alpha$ и сдвигом $\rho$ разрешима тогда и только тогда, когда а и $\rho$ вычислимы.

ДокАзАТЕЛЬство. $\Leftarrow$. Пусть $\alpha$ и $\rho$ вычислимы. Все, кроме, возможно, двух символов последовательности, вычислимы: для вычисления $s_{\alpha, \rho}(n)=$ $\lfloor\alpha(n+1)+\rho\rfloor-\lfloor\alpha n+\rho\rfloor$ нужно с достаточной степенью точности вычислить $\alpha$ и $\rho$, чтобы можно было вычислить $\lfloor\alpha n+\rho\rfloor$ и $\lfloor\alpha(n+1)+\rho\rfloor$, а значит, и $s_{\alpha, \rho}(n)$. Это возможно, если только не окажется, что $\alpha n+\rho$ или $\alpha(n+1)+\rho$ целые все такие $n$ (их не больше двух) мы запоминаем особо и в программу, вычисляющую $s_{\alpha, \rho}$, записываем как отдельный случай.

Далее, ясно, что если последовательность вычислима, и мы для каждого $n$ знаем количество подслов длины $n$ в ней - в данном случае это количество равно $n+1$ (см. теорему 6.1.2 далее) - то множество подслов разрешимо. Отсюда по следствию 3.1.6 получаем, что $\mathrm{MT}\left\langle\mathbb{N},<, s_{\alpha, \rho}\right\rangle$ разрешима.

$\Rightarrow$. Предположим, что теория $\mathrm{MT}\left\langle\mathbb{N},<, s_{\alpha, \rho}\right\rangle$ разрешима. Отсюда следует, что последовательность $s_{\alpha, \rho}$ вычислима. Заметим, что

$$
k_{n}=\sum_{i=1}^{n} s_{\alpha, \rho}(i)=\lfloor\alpha(n+1)+\rho\rfloor-\lfloor\rho\rfloor=\lfloor\alpha(n+1)+\rho\rfloor .
$$

Отсюда $k_{n} \leqslant \alpha(n+1)+\rho<k_{n}+1$, следовательно,

$$
\frac{k_{n}-1}{n+1}<\alpha<\frac{k_{n}+1}{n+1} \text {. }
$$


Это позволяет нам оценить $\alpha$ с точностью $2 /(n+1)$. Таким образом, число $\alpha$ вычислимо, потому что его можно вычислить с любой наперед заданной точностью.

Теперь возможны два случая. Первый случай: $\alpha n+\rho$ при каком-нибудь $n$ является целым числом. Тогда ясно, что число $\rho$ в этом случае вычислимо.

Рассмотрим второй случай, когда $\alpha n+\rho$ не является целым ни при каком $n$. Будем все числа рассматривать по модулю 1 , т. е. не различать числа, отличающиеся на целое слагаемое. Такие числа удобно представлять себе отмеченными на окружности единичной длины, на которой мы в положительную сторону идем против часовой стрелки, а в отрицательную по часовой. Соответствующим образом понимаются и двойные неравенства между такими числами: $a<b<c$, если тройка $(a, b, c)$ ориентирована против часовой стрелки. Заметим, что $s_{\alpha, \rho}(n)=0$, если $0<\alpha n+\rho<-\alpha$, и $s_{\alpha, \rho}(n)=1$, если $-\alpha<\alpha n+\rho<0$. Следовательно, $s_{\alpha, \rho}(n)=0$ при $-\alpha n<\rho<-\alpha(n+1)$ и $s_{\alpha, \rho}(n)=1$ при $-\alpha(n+1)<\rho<\alpha n$.

Чтобы посчитать $\rho$ с точностью $2 \varepsilon$, нужно сначала для достаточно большого $N$ отметить на окружности точки $\alpha k$ для всех $k=0,1,2, \ldots, N$, так чтобы расстояния между соседними отмеченными точками не превышали $2 \varepsilon$. Для этого $\alpha$ должно быть вычислено с точностью $\varepsilon / N$, и расстояния между вычисленными значениями не должны превышать $\varepsilon$. Такое достаточно большое $N$ найдется, так как $\alpha$ иррационально, а при иррациональных $\alpha$ последовательность $\alpha n$ всюду плотна на окружности. После этого, вычислив значения $s_{\alpha, \rho}(k)$ для $k=0,1,2, \ldots, N$, мы сможем ограничить $\rho$ одним из получившихся интервалов длины не более $2 \varepsilon$. Теорема доказана.

Рассмотрим равномерные аналоги утверждений, доказанных в теореме 3.2.2. Предположим, мы хотим построить алгоритм, который, получив на вход числа $\alpha$ и $\rho$, разрешает теорию $\mathrm{MT}\left\langle\mathbb{N},<, s_{\alpha, \rho}\right\rangle$. Если бы существовала процедура, распознающая факт существования такого $n$, что $\alpha n+\rho$ целое, то ее можно было бы использовать так, как описано в доказательстве теоремы 3.2.2. Но известно, что такой процедуры не существует. Однако это еще не доказывает, что желаемого алгоритма не существует. Обратно аналогично: при наличии вышеупомянутой процедуры можно построить алгоритм, который по алгоритму разрешения теории $\mathrm{MT}\left\langle\mathbb{N},<, s_{\alpha, \rho}\right\rangle$ мог бы вычислять $\alpha$ и $\rho$. Но известное нам отсутствие такой процедуры еще не влечет отсутствия желаемого алгоритма. Это порождает следующий вопрос.

ПроблемА 9. 1) Существует ли алгоритм, который, получив алгоритм разрешения теории $\mathrm{MT}\left\langle\mathbb{N},<, s_{\alpha, \rho}\right\rangle$ на вход, может вычислять $\alpha$ и $\rho$ ?

2) Разрешима ли теория $\mathrm{MT}\left\langle\mathbb{N},<, s_{\alpha, \rho}\right\rangle$ относительно алгоритма вычисления $\alpha$ и $\rho$ ?

\section{4. Вычислимость для почти периодических последовательностей}

4.1. Неразрешимость некоторых свойств почти периодических последовательностей. Во многих вопросах, связанных с почти периодическими и с обобщенно почти периодическими последовательностями, естественным 
образом возникает алгоритмическая составляющая: можно ли ту или иную характеристику или то или иное свойство проверять алгоритмически, получая на вход последовательность. Иногда эти вопросы являются эффективными аналогами уже известных результатов, например, теорема 2.2.5 - эффективный аналог теоремы 2.2.4. В настоящем пункте в основном будут рассмотрены результаты, когда ответ на эти вопросы отрицательный. Мы доказываем, что некоторые свойства почти периодичности не имеют эффективных аналогов.

Формально, мы рассматриваем вычислимые операторы на последовательноcmяx. Отметим, что тут возможны два подхода. В первом мы рассматриваем операторы, определенные на всевозможных последовательностях. Такие операторы в процессе вычисления могут обращаться к любым элементам последовательности. Основное свойство вычислимых операторов - непрерывность относительно топологии, индуцированной метрикой $d_{C}$. Другими словами, перед тем, как выдать ответ, оператор может прочитать лишь конечное количество элементов последовательности. Таким образом, для доказательства неразрешимости некоторого свойства таким оператором достаточно показать, что это свойство не является непрерывным.

При втором подходе мы рассматриваем операторы, определенные только на вычислимых последовательностях. На вход оператору подается процедура вычисления последовательности. Однако можно доказать, что при таком определении оператор также будет обладать свойством непрерывности (см. [63]). Поэтому основным для нас будет первый подход, но все результаты настоящего пункта верны и при втором подходе.

Почти никакие осмысленные свойства нельзя распознать, имея на входе только саму последовательность. Например, про $x \in \mathbb{B}^{\mathbb{N}}$ нельзя даже сказать, входит ли в нее символ 1: если алгоритм проверил некоторое конечное количество символов и все они оказались 0 , он не может гарантировать, что далее не встретится 1. Вопрос об эффективности станет более интересным, если мы на вход будем подавать какую-то дополнительную информацию. В случае с обобщенно почти периодическими последовательностями в качестве этой информации естественно взять регулятор почти периодичности. (Ясно, что точно так же можно рассматривать операторы, которым на вход подаются и функции, а не только последовательности.)

Теперь мы видим, что при таком подходе сформулированная выше задача может быть решена эффективно: прочитав первые $f(1)$ символов последовательности, мы можем понять, входит ли в нее 1, а прочитав следующие $f(1)$ символов, сможем сказать даже, входит ли в нее 1 бесконечно много раз. Более того, как следует из теоремы 3.1.5, при таком подходе любое свойство последовательности $x$, выразимое формулой $\varphi(x)$ монадического языка, оказывается разрешимым, если разрешающий алгоритм может использовать $x$.

Следующие несколько теорем - примеры результатов о неэффективности. Особенно интересно сопоставить утверждения теоремы 4.1.1 и теоремы 2.2.5. Теорема 4.1.3 также связана с теоремой 2.2.5. Теоремы 4.1.1, 4.1.2, 4.1.3 и п. 1) предложения 4.1.6 (для класса $\mathscr{A} \mathscr{P}$, что в данном случае несущественно) были впервые объявлены в [49] и доказаны в [12]. Схема доказательства теоре- 
мы 4.1.4, сформулированная для п. 2) предложения 4.1.6, изложена в [64] (здесь мы приводим полное доказательство).

Под сходимостью $f_{n} \rightarrow f$, где $f_{n}, f: \mathbb{N} \rightarrow \mathbb{N}$, будем понимать условие

$$
\forall i \exists n \forall m>n \quad f_{m}(i)=f(i) .
$$

Tеорема 4.1.1 [49], [12]. По $x \in \mathscr{E} \mathscr{A} \mathscr{P}$ u $f \geqslant \mathrm{R}_{x}$ невозможно алгоритмически определять какое-либо $l \geqslant \operatorname{pr}(x)$.

Приведем доказательство этой теоремы, чтобы продемонстрировать используемое во всех теоремах такого типа рассуждение.

Напомним, что последовательность Туэ-Морса можно определить как $\mathbf{t}=$ $\lim _{n \rightarrow \infty} u_{n}$, где последовательность слов $u_{n}$ определяется по индукции: $u_{0}=0$ и $u_{n+1}=u_{n} \bar{u}_{n}$. При этом $\left|u_{n}\right|=2^{n}$. Последовательность $\mathbf{t}$ не содержит кубов. (Подробнее о последовательности t см. п. 1.1. На самом деле, как будет видно из доказательства, нам достаточно даже более слабого утверждения отсутствия кубов специального вида.)

ДОКАЗАТЕЛЬСТВО ТЕОРЕМЫ 4.1.1. Для доказательства достаточно построить $x_{n} \in \mathscr{E} \mathscr{A} \mathscr{P}, x \in \mathscr{A} \mathscr{P}$ с оценками на регуляторы $f_{n} \geqslant \mathrm{R}_{x_{n}}$ и $f \geqslant \mathrm{R}_{x}$, такие что $x_{n} \rightarrow x, f_{n} \rightarrow f$, но $\operatorname{pr}\left(x_{n}\right) \rightarrow \infty$.

Действительно, предположим, указанный в условии теоремы алгоритм существует. Пусть, получив на вход $\langle x, f\rangle$, он выдает число $l \geqslant 0$ (оно может быть произвольным, так как $x \in \mathscr{A} \mathscr{P})$. Во время вычисления $l$ алгоритм прочитал лишь конечное количество символов $x$ и значений $f$, поэтому существует такое $N>l$, что алгоритм не знает $x(k)$ и $f(k)$ для $k>N$ (формально это означает, что на всех входах, отличающихся от $\langle x, f\rangle$ только в позициях $k>N$, алгоритм работает так же, как и на $\langle x, f\rangle)$. Поскольку $\operatorname{pr}\left(x_{n}\right) \rightarrow \infty$, найдется $n$, для которого $\operatorname{pr}\left(x_{n}\right)>N$. Ясно тогда, что на входе $\left\langle x_{n}, f_{n}\right\rangle$ алгоритм будет работать так же, как и на $\langle x, f\rangle$, и, значит, выдаст $l$, но $\operatorname{pr}\left(x_{n}\right)>N>l$.

Положим $x=\mathbf{t}, x_{n}=u_{n} u_{n} u_{n} x$. Тогда $\operatorname{pr}\left(x_{n}\right) \geqslant 2^{n}$. Действительно, если $\operatorname{pr}\left(x_{n}\right)<2^{n}$, то $u_{n} u_{n} x=u_{n} u_{n} u_{n} \bar{u}_{n} \bar{u}_{n} u_{n} \ldots \in \mathscr{A} \mathscr{P}$, и, значит, $u_{n} u_{n} u_{n}$ входит в $x=\mathbf{t}-$ противоречие с бескубностью $\mathbf{t}$.

Осталось показать, что можно выбрать оценку на регуляторы $f_{n}, f$, так что $f_{n} \rightarrow f$. Для этого достаточно найти общий регулятор $g$ для всех $x_{n}$ - потом мы можем увеличить $g$ и добиться того, чтобы $g$ была общей оценкой на регулятор для всех $x_{n}$ и для $x$. Положим $g=4 \mathrm{R}_{x}$. Пусть слово $v,|v|=k$ входит в $x_{n}=$ $u_{n} u_{n} u_{n} x$ бесконечно много раз. Возьмем отрезок $[i, j]$ последовательности $x$ длины $4 \mathrm{R}_{x}(k)$ и покажем, что $v$ в него входит. Если $j \geqslant 3 \cdot 2^{n}+\mathrm{R}_{x}(k)$, то $v$ встречается на отрезке $x\left[3 \cdot 2^{n}, 3 \cdot 2^{n}+\mathrm{R}_{x}(k)\right]$ (по определению регулятора). Иначе $j<3 \cdot 2^{n}+\mathrm{R}_{x}(k)$, откуда $i \leqslant 3 \cdot 2^{n}-3 \mathrm{R}_{x}(k)$. Но $i \geqslant 0$, значит, в этом случае $\mathrm{R}_{x}(k) \leqslant 2^{n}=\left|u_{n}\right|$. Таким образом, $x_{n}\left[i, i+\mathrm{R}_{x}(k)\right]$ целиком содержится в $u_{n} u_{n}$. Но $u_{n} u_{n}$ входит в $x$, и, значит, $x_{n}\left[i, i+\mathrm{R}_{x}(k)\right]$ входит в $x$, поэтому $v$ входит в $x\left[i, i+\mathrm{R}_{x}(k)\right]$, так как $v$ входит в $x$ бесконечно много раз, а значит, входит в любое подслово $x$ длины $\mathrm{R}_{x}(k)$.

Однако $g$ - еще не искомая. Необходимо проследить за словами, которые встречаются в $x_{n}$ конечное количество раз. Но ясно, что если какое-то $v$ входит в $x_{n}$ конечное количество раз, то $|v|=k>2^{n}$ (иначе $v$ входит в блок 
из двух последовательных слов $u_{n}$ или $\bar{u}_{n}$, а значит, и в $x$ ), т. е. такое может быть лишь для конечного множества различных $n$. Поэтому, рассмотрев все ситуации, когда слова фиксированной длины $k$ входят в какие-то $x_{n}$ конечное количество раз, нам, возможно, придется увеличить значение $g(k)$, но лишь конечное количество раз. Значит, искомая оценка регуляторов найдется. Теорема доказана.

Как мы уже отмечали в п. 1.5, имеем $\mathscr{E} \mathscr{A} \mathscr{P} \subsetneq \mathscr{G} \mathscr{A} \mathscr{P}$ [47]. Можно показать, что эти классы невозможно разделять эффективно.

Tеорема 4.1.2 [49], [12]. По $x \in \mathscr{G} \mathscr{A} \mathscr{P}$ u $f \geqslant \mathrm{R}_{x}$ невозможно алгоритмически определять, верно ли, что $x \in \mathscr{E} \mathscr{A} \mathscr{P}$.

Следующее утверждение по существу говорит о том, что даже приписав к почти периодической последовательности один символ, мы, вообще говоря, уже не можем проверять, является ли она по-прежнему почти периодической.

Tеорема 4.1.3 [49], [12]. По $x \in \mathscr{E} \mathscr{A} \mathscr{P}, f \geqslant \mathrm{R}_{x}$ u некоторому $l \geqslant \operatorname{pr}(x)$ невозможно алгоритмически находить $\operatorname{pr}(x)$.

Следующий довольно общий результат был изначально мотивирован вопросом о регулярности множества автоматных последовательностей - см. предложение 4.1.6, п. 2) и следствие 4.3.2, п. 2), доказанные в [64].

Теорема 4.1.4. Пусть $\mathscr{M}$ - счетное множество последовательностей, содержащее все периодические последовательности. Тогда по $x \in \mathscr{G} \mathscr{A} \mathscr{P} u$ $f \geqslant \mathrm{R}_{x}$ невозможно алгоритмически определять, верно ли, что $x \in \mathscr{M}$.

ДокАЗАТЕЛЬСтво. Достаточно построить последовательность $x_{n}$ бесконечных слов и бесконечное слово $x$, так что $x_{n}$ стремится к $x$, все слова $x_{n}, x$ обобщенно почти периодичны и имеют общую оценку на регулятор почти периодичности $g \geqslant \mathrm{R}_{x_{n}}, g \geqslant \mathrm{R}_{x}$, причем все $x_{n} \in \mathscr{M}$, а $x \notin \mathscr{M}$. Действительно (повторим основную схему рассуждения еще раз), предположим, алгоритм из условия теоремы (распознающий принадлежность множеству $\mathscr{M}$ обобщенно почти периодических последовательностей) существует. Подадим ему на вход $x$ и $g$. Он выдаст отрицательный ответ, так как $x \notin \mathscr{M}$. Во время своей работы, перед тем как выдать ответ, алгоритм прочитал лишь какое-то конечное количество символов из $x$. Последовательность $x_{n}$ стремится к $x$, значит, те же самые символы, которые алгоритм успел прочитать в $x$, стоят на тех же местах в каком-то $x_{m}$ для некоторого $m$. Значит, тот же самый отрицательный ответ алгоритм должен выдать и на $x_{m}$ и $g$ - противоречие, так как $x_{m} \in \mathscr{M}$.

Построим теперь нужные $x_{n}, x$.

В п. 2.4 мы описали универсальный метод построения почти периодических последовательностей. Напомним один из его вариантов.

Последовательность $\left\langle B_{n}, l_{n}\right\rangle$, где $B_{n}$ - непустое множество непустых слов в фиксированном конечном алфавите $A, l_{n}$ - натуральные числа, является $A$ - $\mathscr{A} \mathscr{P}$-схемой, если для нее выполнено для любого $n \in \mathbb{N}$ :

1) все слова из $B_{n}$ имеют длину $l_{n}$;

2) любое слово $u \in B_{n+1}$ представимо в виде $u=v_{1} v_{2} \ldots v_{k}$, где $v_{i} \in B_{n}$, и для любого $w \in B_{n}$ существует $i$ такое, что $v_{i}=w$. 
Последовательность $x \mathscr{A} \mathscr{P}$-порождена $A$ - $\mathscr{A} \mathscr{P}$-схемой, если для любых $i$ и $n$ имеем $x\left[i l_{n},(i+1) l_{n}-1\right] \in B_{n}$ (далее приставки $\mathscr{A} \mathscr{P}$ - и $A$ - $\mathscr{A} \mathscr{P}$ - мы опускаем).

Как мы уже говорили, каждая схема порождает какую-то последовательность, и каждая последовательность, порожденная схемой, является почти периодической. Более того, каждая почти периодическая последовательность порождается некоторой схемой.

Теперь мы усилим главное условие на $B_{n}$, а именно, будем рассматривать схемы, для которых верно:

(*) для любого $n>1$ каждое $u \in B_{n}$ имеет вид $u=v_{1} v_{2} \ldots v_{k}$, где $v_{i} \in B_{n-1}$, причем для каждых $w_{1}, w_{2} \in B_{n}$ найдется $i<k$ такое, что $v_{i} v_{i+1}=w_{1} w_{2}$.

Отметим, что так мы теряем свойство универсальности: очевидно, не каждая почти периодическая последовательность может быть порождена схемой, удовлетворяющей условию $(*)$.

Лемма 4.1.5. Существует схема, для которой выполнено условие (*), и которая порождает континуум различных последовательностей.

ДокАЗАтЕЛьство. Утверждение леммы почти очевидно, но тем не менее докажем его и построим необходимую $\mathscr{A} \mathscr{P}$-схему.

Определим рекурсивно схему $\left\langle l_{n}, B_{n}\right\rangle$ : положим $l_{0}=1$ и $B_{0}=\{0,1\}$, и дальше для каждого $n \geqslant 1$ положим $l_{n}=\left(\# B_{n-1}\right)^{2} l_{n-1}$. Также положим $B_{n}$ состоящим из всех слов длины $l_{n}$, которые удовлетворяют условию $(*)$.

Теперь объясним, почему эта схема порождает континуум различных последовательностей. Начнем строить какую-нибудь последовательность, порождаемую этой схемой. Для этого выберем последовательность слов $w_{i}$ рекурсивно следующим образом: слово $w_{0}$ из множества $B_{0}$ выберем произвольно, а далее слово $w_{i}$ для $i \geqslant 1$ будем выбирать произвольно из множества $B_{i}$, так чтобы оно являлось продолжением слова $w_{i-1}$. Заметим, что длина $l_{i}$ выбрана достаточной для того, чтобы для любого $v \in B_{i-1}$ можно было найти более одного слова из $B_{i}$, являющегося продолжением $v$. Это значит, что каждый раз, когда мы выбираем $w_{i}$ для какого-нибудь $i$, у нас есть хотя бы два различных варианта, независимо от всех предыдущих выборов. Значит, мы можем выбрать последовательность слов $w_{i}$ континуумом различных способов. Осталось только заметить, что при любом таком выборе предельное бесконечное слово $\lim _{i \rightarrow \infty} w_{i}$ порождено схемой $\left\langle l_{n}, B_{n}\right\rangle$ по построению. Лемма доказана.

Как следует из этой леммы, среди порожденных такими усиленными схемами найдутся последовательности, не принадлежащие $\mathscr{M}$, так как $\mathscr{M}$ счетно (это единственное место, где используется счетность $\mathscr{M}$ ). Возьмем одну из них $-x$, порожденную некоторой схемой $\left\langle B_{n}, l_{n}\right\rangle$.

Определим $p_{n}=x\left[0, l_{n}\right]$. Таким образом, $p_{n} \in B_{n}$ и $\lim _{n \rightarrow \infty} p_{n}=x$. Положим $x_{n}=p_{n} p_{n} p_{n} \ldots \in \mathscr{P}$. Ясно, что $x_{n} \rightarrow x$, кроме того, все $x_{n} \in \mathscr{M}$. Осталось выбрать общий регулятор $f$ для всех $x_{n}$. Увеличив его потом, если нужно, сделаем так, чтобы он подходил и для $x$.

Сейчас мы получим конечное (для каждого $k$ ) количество требований вида $f(k) \geqslant \alpha$, после чего в качестве $f(k)$ можем взять максимум по всем таким $\alpha$.

Пусть $v=x_{n}[i, j],|v|=k$ (это сразу означает, что $v$ входит в $x_{n}$ бесконечно много раз, так как $\left.x_{n} \in \mathscr{P}\right)$. Неравенство $k \geqslant l_{n-1}$ может выполняться лишь 
для конечного количества различных $n$ (при фиксированном $k$ ), что дает лишь конечное количество условий на $f(k)$. Теперь можно считать, что $k<l_{n-1}$. Возьмем такое $t$, что $l_{t-1}<k \leqslant l_{t}$ (важно, что $t$ не зависит от $n$ и однозначно определяется по $k$ ). Тогда $t \leqslant n-1$. Существует $m$ такое, что $m l_{t} \leqslant i$ и $j \leqslant$ $(m+2) l_{t}-1$, т. е. $v$ содержится в некотором $a b$, где $a, b \in B_{t}$, и значит, благодаря свойству $(*), v$ входит в любое $c \in B_{t+1}$. Но на каждом отрезке длины $2 l_{t+1}$ последовательности $x_{n}$ найдется вхождение какого-нибудь $c \in B_{t+1}$ (целиком входящего в какое-то $\left.p_{n}\right)$, а значит, и вхождение $v$. Таким образом, достаточно $f \geqslant 2 l_{t+1}$. Теорема 4.1 .4 доказана.

ПреДЛОЖеНИЕ 4.1.6. 1) [49], [12] По $x \in \mathscr{G} \mathscr{A} \mathscr{P} u f \geqslant \mathrm{R}_{x}$ невозможно алгоритмически определять, верно ли, что $x \in \mathscr{P}$.

2) [64] По $x \in \mathscr{G} \mathscr{A} \mathscr{P}$ u $f \geqslant \mathrm{R}_{x}$ невозможно алгоритмически определять, верно ли, что $x$ автоматна.

3) По $x \in \mathscr{G} \mathscr{A} \mathscr{P}$ u $f \geqslant \mathrm{R}_{x}$ невозможно алгоритмически определять, верно ли, что $x$ морфическая.

Из конструкции в доказательстве теоремы 4.1.4 следует существование бесконечного количества периодических последовательностей $x_{n}$ с общей верхней оценкой на регулятор почти периодичности. Эта серия примеров любопытна тем, что одна естественная мера повторяемости в этих последовательностях оценка на регулятор почти периодичности - постоянна, тогда как другая такая мера - период последовательности - может быть сколь угодно большой. По-другому, это замечание можно сформулировать так: если естественным образом обобщить понятие регулятора почти периодичности на конечные слова и взять последовательность слов $p_{n}-$ периодов $x_{n}$, то это будет пример сколь угодно длинных конечных слов с общей оценкой сверху на регулятор почти периодичности.

4.2. Связь с монадическими теориями. Любопытным образом оказывается теперь, что из вышеперечисленных результатов и из теоремы 3.1.5 можно получить следствия в терминах монадических теорий следующим образом.

СлЕДСТВИЕ 4.2.1. Не существует такой формулы $\varphi(x, l)$ в монадическом языке, которая при $x \in \mathscr{E} \mathscr{A} \mathscr{P}$ истинна, только если $l \geqslant \operatorname{pr}(x)$, причем при каждом $x \in \mathscr{E} \mathscr{A} \mathscr{P}$ хотя бы одно такое $l$, что $\varphi(x, l)$ истинна, найдется.

ДоказАТЕЛьство. Пусть $x \in \mathscr{E} \mathscr{A} \mathscr{P}$, и пусть существует формула $\varphi(x, l)$ из условия теоремы. Тогда по $x$ и какому-то $f \geqslant \mathrm{R}_{x}$, можно перебором найти $l \geqslant \operatorname{pr}(x)$. Для этого нужно перебирать подряд все натуральные числа $n$ и проверять, верно ли $\varphi(x, n)$ - это можно проверить по теореме 3.1.5. Таким образом, это дало бы алгоритмический способ находить $l \geqslant \operatorname{pr}(x)$ по $x$ и $f \geqslant \mathrm{R}_{x}$ - противоречие с теоремой 4.1.1. Следствие доказано.

Аналогичным образом получаются и приведенные ниже следствия. Скажем, что свойство $P$ выразимо при $x \in C$ в теории $\mathrm{MT}\langle\mathbb{N},<, x\rangle$, если существует такая формула $\varphi(x)$ в монадическом языке, которая в ограничении на множество $C$ выражает свойство $P$.

СлеДСТвиЕ 4.2.2. Если $x \in \mathscr{G} \mathscr{A} \mathscr{P}$, то в теории $\mathrm{MT}\langle\mathbb{N},<, x\rangle$ невыразимо свойство $x \in \mathscr{E} \mathscr{A} \mathscr{P}$. 
ДокАЗАТЕЛЬСтво. Следует из теоремы 4.1.2.

СлеДСтвие 4.2.3. Если $x \in \mathscr{E} \mathscr{A} \mathscr{P}$, то в теории $\mathrm{MT}\langle\mathbb{N},<, x\rangle$ с дополнительной константой, равной какому-то $l \geqslant \operatorname{pr}(x)$, невыразимо число $\operatorname{pr}(x)$.

ДокАЗАтельство. Следует из теоремы 4.1.3.

СлЕДСТвиЕ 4.2.4. 1) Если $x \in \mathscr{G} \mathscr{A} \mathscr{P}$, то в теории $\mathrm{MT}\langle\mathbb{N},<, x\rangle$ невыразимо свойство $x \in \mathscr{P}$.

2) Если $x \in \mathscr{G} \mathscr{A} \mathscr{P}$, то в теории $\mathrm{MT}\langle\mathbb{N},<, x\rangle$ невыразимо свойство " $x$ автоматная последовательность".

3) Если $x \in \mathscr{G} \mathscr{A} \mathscr{P}$, то в теории $\mathrm{MT}\langle\mathbb{N},<, x\rangle$ невыразимо свойство " $x$ морфическая последовательность".

ДокАЗАТЕЛЬСтво. Следует из предложения 4.1.6.

О связанном с результатами этого пункта понятии самовыразимости см. [65].

4.3. Нерегулярность некоторых множеств последовательностей. Теперь мы покажем, как, используя теоремы этого раздела, можно доказать про некоторые множества последовательностей, что они не распознаются конечным автоматом (имеется в виду автомат Бюхи или автомат Мюллера, см. п.3.1), т. е. не являются регулярными. Возможно, у этих фактов есть и более прямое доказательство. В изложении этого пункта мы в существенной степени следуем работе [64].

Как утверждает теорема 3.1.5, по обобщенно почти периодической последовательности, какой-нибудь оценке сверху на ее регулятор и автомату Бюхи можно алгоритмически определять, принимает ли данный автомат Бюхи данную последовательность. Говоря более точно, существует алгоритм, который, получив на вход оракул для последовательности $x \in \mathscr{G} \mathscr{A} \mathscr{P}$, оракул для некоторой функции $g \geqslant \mathrm{R}_{x}$ и автомат Бюхи $F$, выдает ответ, верно ли, что $F$ принимает $x$.

Предположим, что некоторое множество $\mathscr{M}$ последовательностей является регулярным, т. е. существует принимающий его автомат Бюхи. Тогда по теореме 3.1 .5 существует алгоритм, который по обобщенно почти периодической последовательности и оценке сверху на ее регулятор определяет, принадлежит ли эта последовательность множеству $\mathscr{M}$.

Таким образом, для того чтобы показать, что множество $\mathscr{M}$ нерегулярно, достаточно показать, что не существует алгоритма, который по оракулу для обобщенно почти периодической последовательности и для какой-то оценки сверху на ее регулятор определяет принадлежность этой последовательности к множеству $\mathscr{M}$. (Обобщенно почти периодические последовательности в этом утверждении можно заменить на почти периодические.)

Так, из теоремы 4.1.2 и предложения 4.1.6 можно получить следующие результаты для фиксированного алфавита.

СледСТВиЕ 4.3.1. Множество $\mathscr{E} \mathscr{A} \mathscr{P}$ не регулярно.

СлЕДСТвиЕ 4.3.2. 1) Множество Я не регулярно.

2) Множество автоматных последовательностей не регулярно.

3) Множество морфических последовательностей не регулярно. 
В качестве естественного продолжения можно сформулировать следующую гипотезу.

ГипотезА 10. Множества $\mathscr{A} \mathscr{P}, \mathscr{G} \mathscr{A} \mathscr{P}, \mathscr{R}, \mathscr{E} \mathscr{R}$ не регулярны.

Скорее всего, в этих случаях также можно применить технику, продемонстрированную в этом пункте.

\section{5. Автоматные и морфические последовательности. Соотношения с почти периодичностью}

5.1. Автоматные последовательности. Любая заключительно периодическая последовательность может быть порождена машиной с конечной памятью: достаточно иметь информацию о предпериоде и периоде последовательности. И наоборот, любая машина с конечной памятью, печатающая символы конечного алфавита, порождает заключительно периодическую последовательность. Действительно, во время ее работы в какой-то момент конфигурация машины полностью совпадет с какой-то из уже встречавшихся, и так начнется период в выходной последовательности.

Кобхэм в [55] предложил другой способ использования конечных автоматов для определения последовательностей. В его определении автоматам подаются на вход моменты времени.

Рассмотрим конечный автомат, действующий на словах в алфавите $\Sigma_{k}=$ $\{0,1, \ldots, k-1\}$. Каждому состоянию автомата соответствует буква в некотором другом алфавите $A$ (разным состояниям могут соответствовать одинаковые буквы). Автомат действует так: получает на вход слово в алфавите $\Sigma_{k}$, производит вычисления и выдает ту букву алфавита $A$, которая соответствует последнему состоянию в вычислении. Последовательность $x$ букв алфавита $A$ называется $k$-автоматной, если существует конечный автомат вышеуказанного вида, который, будучи запущенным на числе $n$, записанном в $k$-ичной системе счисления, выдает букву $x(n)$. Когда ясно или неважно, о каком $k$ идет речь, приставку " $k$-" мы будем опускать.

Примером 2-автоматной последовательности является последовательность Туэ-Морса (см. наше второе определение из п. 1.1). Действительно, несложно придумать автомат из двух состояний, который по записи числа в двоичной системе счисления вычисляет четность его суммы цифр.

Теперь опишем другой естественный подход, приводящий к тому же понятию.

В этом разделе мы рассматриваем морфизмы (см. определения в п. 1.3) вида $A^{*} \rightarrow A^{*}$. Пусть $\varphi(s)=s u$ для некоторых $s \in A, u \in A^{*}$. Тогда для всех натуральных $m<n$ слово $\varphi^{n}(s)$ начинается со слова $\varphi^{m}(s)$, так что можно корректно определить $\varphi^{\infty}(s)=\lim _{n \rightarrow \infty} \varphi^{n}(s)=s u \varphi(u) \varphi^{2}(u) \varphi^{3}(u) \ldots$ Если $\varphi^{n}(u) \neq \Lambda$ для всех $n$, то $\varphi^{\infty}(s)$ бесконечна. В этом случае говорят, что морфизм $\varphi$ продолжаем на $s$. Последовательности вида $h\left(\varphi^{\infty}(s)\right)$, где $h: A \rightarrow B-$ кодирование, называются морфическими, вида $\varphi^{\infty}(s)$ - чисто морфическими.

Например, последовательность Туэ-Морса является чисто морфической, поскольку порождается итерациями морфизма $0 \rightarrow 01,1 \rightarrow 10$ на символе 0 . 
Теорема 5.1.1 [55], [42].Последовательность $k$-автоматна тогда и только тогда, когда она является морфической, полученной из $k$-равномерного морфизма.

Благодаря теореме 5.1.1 мы имеем два эквивалентных определения автоматных последовательностей.

Одним из обобщений последовательности Туэ-Морса на произвольный алфавит являются последовательности Пруэ, по существу введенные в [23] (см. также п. 1.1), которые были названы в честь Пруэ в [66], и активно после этого изучались (например, см. [67], [68]).

Определим морфизм $\varphi:\{0, \ldots, k-1\}^{*} \rightarrow\{0, \ldots, k-1\}^{*}$ следующим образом:

$$
\begin{aligned}
& \varphi(0)=0123 \ldots(k-2)(k-1), \\
& \varphi(1)=123 \ldots(k-2)(k-1) 0, \\
& \varphi(2)=23 \ldots(k-2)(k-1) 01, \\
& \ldots \ldots \ldots \ldots \ldots \ldots \ldots \ldots \ldots \\
& \varphi(k-1)=(k-1) 0123 \ldots(k-2) .
\end{aligned}
$$

Другими словами, $(\varphi(i))(j)=i+j(\bmod k)$ для $0 \leqslant i, j \leqslant k-1$. Последовательность $\mathbf{t}_{k}=\varphi^{\infty}(0)$ называется последовательностью Пруэ. Видно, что $\mathbf{t}_{k}$ является $k$-автоматной. Это можно увидеть и из другого, эквивалентного определения этой последовательности: $\mathbf{t}_{k}(n)$ равно сумме цифр по модулю $k$ числа $n$, записанного в системе счисления с основанием $k$ (см. также п. 1.1).

Из определения видно, что автоматная последовательность может быть конечно описана - достаточно либо задать конечный автомат, который ее порождает, либо задать порождающий равномерный морфизм, букву, с которой надо начинать итерировать, и кодирование. Это значит, в частности, что можно корректно формулировать вопросы о разрешимости различных свойств автоматных последовательностей.

В контексте этой статьи для нас наиболее интересным представляется сравнивать различные определения последовательностей, близких к периодическим. Ясно, например, что существуют почти периодические последовательности, не являющиеся автоматными - почти периодических последовательностей континуум, в то время как автоматных, очевидно, счетное количество. И наоборот, существуют автоматные последовательности, не являющиеся почти периодическими. Действительно, последовательность

$$
011010001000000010000000000000001 \ldots,
$$

у которой на каждом $2^{n}$-м месте стоит 1 , а на остальных 0 , является 2 -автоматной (легко построить автомат, отделяющий числа вида $100 \ldots 0$ в двоичной системе счисления от всех остальных), но не является почти периодической.

Естественно задать вопрос о разрешимости свойства почти периодичности для автоматных последовательностей. Оказывается, на него можно ответить положительно.

ТЕОРема 5.1.2 [69], [70]. Существует полиномиалъный по времени алгоритм, который по автоматной последовательности определяет, является ли она почти периодической. 
Интересно следующее свойство замкнутости для автоматных последовательностей.

ТеОРема 5.1.3 [55]. Множество $k$-автоматных последовательностей замкнуто относительно равномерных конечно-автоматных преобразований.

О конечно-автоматных преобразованиях см. п. 2.2. Доказательство теоремы 5.1.3 можно найти также в [42].

ПроБЛЕмА 11. Сохраняется ли множество $k$-автоматных последовательностей под действием произвольных (не обязательно равномерных) морфизмов? Под действием произвольных (не обязательно равномерных) конечно-автоматных преобразований?

5.2. Морфические последовательности. В одном из определений автоматных последовательностей используются морфические последовательности, порожденные равномерными морфизмами. В качестве естественного обобщения можно рассматривать морфические последовательности, порожденные морфизмами произвольного вида.

Класс морфических последовательностей, очевидно, шире класса автоматных последовательностей. Например, последовательность Фибоначчи $\mathbf{f}=0100101001001 \ldots$ (см. п. 1.2) является чисто морфической, порожденной морфизмом $0 \rightarrow 01,1 \rightarrow 0$, но не является автоматной.

Ясно также, что множество морфических последовательностей находится в общем положении с множеством почти периодических последовательностей. Как и в п. 5.1 (см. теорему 5.1.2), возникает естественный вопрос о разрешимости почти периодичности для морфических последовательностей. Однако в этом случае проблема до сих пор не решена.

ГиПотезА 12. Существует алгоритм, который по морфической последовательности определяет, является ли она почти периодической.

Интересно сравнить гипотезу 12 с предложением 4.1.6.

В одном из частных случаев - случае автоматных последовательностей ответ на вопрос о разрешимости положительный (теорема 5.1.2). Ответ положительный и в другом частном случае - чисто морфических последовательностей.

ТЕОРема 5.2.1 [69], [70]. Существует полиномиалъный по времени алгоритм, который по чисто морфической последовательности определяет, является ли она почти периодической.

Во многом свойства морфических последовательностей проще свойств почти периодических. Например, имеет место следующий результат (о монадических теориях см. п. 3.1).

ТеОРема 5.2.2 [62]. Монадическая теория морфической последовательности разрешима.

Теорема 5.2.2 сформулирована и доказана в [62], однако несложно следует и из более ранних результатов. Действительно, в [71] доказано следующее утверждение. 
ТеОРема 5.2.3 [71]. Множество морфических последовательностей замкнуто относителъно конечно-автоматных преобразований.

О конечно-автоматных преобразованиях см. п. 2.2. Доказательство теоремы 5.2.3 можно найти также в [42]. Из доказательства несложно видеть, что утверждение этой теоремы эффективно, т. е. по конечно-автоматному преобразователю $M$ и описанию морфической последовательности $x$ можно получить описание морфической последовательности $M(x)$. Ясно также, что существует (полиномиальный) алгоритм, который по любому символу и морфической последовательности определяет, входит ли этот символ в последовательность бесконечное количество раз (доказательство можно найти в [70]). По следствию 3.1.4 получаем, что монадическая теория морфической последовательности разрешима.

\section{6. Подслова и сложность почти периодических последовательностей}

6.1. Подсловная сложность. Подсловной сложностью $\mathrm{p}_{x}(n)$ последовательности $x$ называется количество слов длины $n$, входящих в $x$.

Подсловная сложность - естественная и просто определяемая характеристика последовательностей, это понятие широко изучалось, см. обзоры [72], [73] или [42; гл. 10].

Следующий известный результат в терминах подсловной сложности характеризует периодические последовательности.

ПрЕДЛОЖЕНИЕ 6.1 .1 [5]. 1) Если для некоторого $n$ верно $\mathrm{p}_{x}(n) \leqslant n$, то последовательность $x$ заключительно периодическая.

2) Последовательность $x$ заключительно периодическая тогда и только тогда, когда ее подсловная сложность ограничена, т.е. найдется такая константа $C$, что $\mathrm{p}_{x}(n) \leqslant C$ для всех $n$.

ДокАзАтЕльство. 1) Возможны два случая. Если $\mathrm{p}_{x}(1)=1$, то это означает, что в последовательность входит только одна буква, и, тем самым, она периодична. Если $\mathrm{p}_{x}(1) \geqslant 2$, то, поскольку функция подсловной сложности не убывает, найдется такое $k$, что $1 \leqslant k<n$ и $\mathrm{p}_{x}(k)=\mathrm{p}_{x}(k+1)$. Отсюда следует, что каждое слово длины $k$, входящее в последовательность, является началом ровно одного слова длины $k+1$, входящего в последовательность. Значит, каждый следующий символ последовательности, начиная с $(k+1)$-го, однозначно определяется предыдущими $k$, и последовательность периодична с этого момента.

2) Пусть последовательность $x$ заключительно периодическая, и длина ее периода равна $p$, а длина предпериода равна $q$. Пусть $n \geqslant p-$ некоторое число. Ясно, что различных слов длины $n$, входящих целиком в периодическую часть $x[q, \infty)$, не больше $p$, а слов длины $n$, “задевающих" предпериод $x[0, q-1]$, не больше $q$. Отсюда $\mathrm{p}_{x}(n) \leqslant p+q$ для $n \geqslant q$.

Если последовательность имеет ограниченную подсловную сложность, то ее заключительная периодичность следует из п. 1). Предложение доказано. 
Последовательности Штурма можно охарактеризовать в терминах подсловной сложности в точности как последовательности $x$, для которых $\mathrm{p}_{x}(n)=n+1$ для любого $n$. Таким образом, последовательности Штурма - это последовательности с минимальной возможной для апериодических последовательностей подсловной сложностью.

Приведем и еще одну характеризацию последовательностей Штурма в терминах подслов. Множество слов $X$ называется сбалансированным, если для любых слов $u, v \in X$ одинаковой длины имеем $\left.|| u\right|_{1}-|v|_{1} \mid \leqslant 1$. Конечное слово или бесконечная последовательность называется сбалансированным/ой, если множество его/ее подслов сбалансированно.

Доказательство следующей теоремы, видимо, уже относящейся к фольклору, можно найти, например, в [38].

Теорема 6.1.2. Для последовательности $x$ следующие условия эквивалентны:

(i) $x$ имеет подсловную сложность $p_{x}(n)=n+1$;

(ii) $x$ сбалансированная и не заключительно периодическая;

(iii) $x$ механическая иррачиональная.

Таким образом, благодаря теореме 6.1.2 мы имеем три эквивалентных определения последовательностей Штурма.

В работе [74] рассмотрено понятие квазиштурмовых последовательностей (в произвольном конечном алфавите) - таких, у которых для некоторых $k$ и $n_{0}$ подсловная сложность равна $\mathrm{p}_{x}(n)=n+k$ для всех $n \geqslant n_{0} . \mathrm{B}$ [74] доказано, что такие последовательности всегда имеют вид $w \psi(x)$, где $w$ - некоторое слово, $x$ - последовательность Штурма над некоторым алфавитом $\{a, b\}, \psi-$ непериодический морфизм, т. е. такой, что $\psi(a b) \neq \psi(b a)$.

В [75] рассмотрено обобщение понятия сбалансированной последовательности на произвольный конечный алфавит и получена характеризация таких последовательностей в терминах динамических систем.

Также интересно понять, что можно сказать про подсловную сложность морфических последовательностей. В серии работ, завершающейся работой [76], доказано, что подсловная сложность чисто морфической последовательности может удовлетворять одной из пяти следующих асимптотик: $O(1), \Theta(n)$, $\Theta(n \log \log n), \Theta(n \log n), \Theta\left(n^{2}\right)$. Про подсловную сложность морфических последовательностей произвольного вида долгое время было ничего неизвестно. В [77] показано, что существуют примеры морфических последовательностей с подсловной сложностью вида $\Theta\left(n^{1+\frac{1}{k}}\right)$ для каждого $k \in \mathbb{N}$. Наконец, в [78] сформулировано и в [79] будет доказано следующее.

Теорема 6.1.3 [78], [79]. Подсловная сложность морфической последовательности имеет один из следующих видов: $O(n \log n), \Theta\left(n^{1+1 / k}\right)$ для некоторого $k \in \mathbb{N}$. Каждый из указанных классов непуст.

Таким образом, для полного описания возможных асимптотик подсловной сложности морфической последовательности осталось разобраться подробнее со случаем $O(n \log n)$. Можно сформулировать следующую гипотезу. 
ГиПотезА 13 [78], [79]. Подсловная сложность морфической последовательности имеет один из следующих видов:

$$
O(1), \quad \Theta(n), \quad \Theta(n \log \log n), \quad \Theta(n \log n), \quad \Theta\left(n^{1+\frac{1}{k}}\right)
$$

для некоторого $k \in \mathbb{N}$. Каждый из указанных классов непуст.

Оказывается, про подсловную сложность морфической последовательности можно сказать гораздо больше, если наложить дополнительные условия.

ТЕОрема 6.1 .4 [70]. Подсловная сложность морфической почти периодической последовательности не более чем линейна.

См. также следствие 6.2.4.

В дальнейшем представляется интересным продолжить изучение подсловной сложности морфических последовательностей, в том числе морфических последовательностей с разными дополнительными ограничениями.

Обратимся к автоматным последовательностям. Близкими к периодическим автоматные последовательности можно назвать и еще по одной причине. Для последовательностей в алфавите из $m$ символов подсловная сложность может варьироваться от 1 до $m^{n}$. Как доказано в [5] (см. предложение 6.1.1), подсловная сложность последовательности ограничена тогда и только тогда, когда последовательность является заключительно периодической. Оказывается, что подсловная сложность автоматных последовательностей тоже не слишком велика.

ТЕОРема 6.1.5 [55]. Подсловная сложность автоматной последовательности не более чем линейна.

Тем не менее, теорема 6.4.6 показывает, что автоматные последовательности могут быть достаточно далеки от периодических.

Обобщение подсловной сложности - арифметическая сложность - было введено в [80] и далее активно изучалось (см., например, [81], [82] и др.) Арифметическая сложность последовательности $x$ - это количество слов $u$ длины $n$, для которых найдутся некоторые $i, d$ такие, что $x(i) x(i+d) x(i+2 d) \ldots$ $x(i+(n-1) d)=u$.

\section{2. Нормальные числа и гипотеза Бореля.}

ПроБлемА 14. Является ли десятичная запись числа $\sqrt{2}$ почти периодической последовательностью? Является ли она морфической? Автоматной?

По всей видимости, проблема 14 очень сложная. Ниже даны некоторые аргументы в пользу такой точки зрения. В изложении этого пункта мы в основном следуем обзорной статье [83]. Для некоторых теорем мы приводим упрощенные ослабленные формулировки. Подробнее см. [83].

Для простоты все действительные числа в этом пункте будем считать лежащими на интервале $[0,1)$. Пусть $x$ - действительное число, $b \in \mathbb{N}$. Запишем $x$ в системе счисления с основанием $b: x=0, x(0) x(1) x(2) \ldots$. Последовательность $x(0) x(1) x(2) \ldots$ в алфавите $\Sigma_{b}=\{0,1, \ldots, b-1\}$ обозначим тогда $x_{b}$.

Назовем действительное число $x$ нормальным, если оно обладает следующим свойством: для любого $b \in \mathbb{N}$ все слова длины $n$ в алфавите $\Sigma_{b}$ входят 
в последовательность $x_{b}$ бесконечно много раз с одинаковой частотой $1 / b^{n}$. Борель доказал, что почти все (по мере Лебега) действительные числа являются нормальными [84]. Таким образом, нормальность можно воспринимать как свойство, которому удовлетворяет любое случайно взятое число.

Напомним, что действительное число называется алгебраическим, если оно является корнем некоторого многочлена от одной переменной с целыми коэффициентами. Верно ли, что алгебраические числа не случайны? Борель предположил, что ответ отрицателен, в следующем смысле.

ГиПотЕЗА 15 (Борель, [85]). Любое алгебраическое иррачиональное число является нормальным.

Эта гипотеза по-прежнему остается недоказанной. В частности, из гипотезы Бореля следует, что десятичная запись числа $\sqrt{2}$ (как и запись по любому основанию, большему 1 , любого алгебраического иррационального числа) не является почти периодической, поскольку для любой почти периодической последовательности (в алфавите хотя бы из двух букв) есть слова, которые в нее не входят (например, слишком длинные отрезки из одного некоторого символа). Но доказать это, по всей видимости, довольно сложно.

Не доказан и следующий, более слабый вариант гипотезы Бореля.

ГИПОТЕЗА 16. Для любого $b \geqslant 3$ и любого алгебраического иррачионалъного числа $x$ каждъй символ алфавита $\Sigma_{b}$ входит в $x_{b}$ хотя бы один раз.

Также не известно ни одного явного примера тройки $x, a, b$, где $x$ - алгебраическое иррациональное число, $b$ - натуральное число, $a \in \Sigma_{k}$, для которых можно доказать, что $a$ входит в $x_{b}$ бесконечно много раз.

Пытаясь подойти к гипотезе Бореля, можно для различных классов последовательностей доказывать, что ни одна последовательность из этого класса не может быть записью алгебраического иррационального числа. Следующая ослабленная формулировка гипотезы Бореля также не доказана. Напомним, что $\mathrm{p}_{y}$ обозначает подсловную сложность последовательности $y$.

ГиПотезА 17. Если $x$ - алгебрачческое иррационалъное число, и $b \in \mathbb{N}$, то $\mathrm{p}_{x_{b}}(n)=b^{n}$.

Даже и эта гипотеза, по всей видимости, является очень сложной. Рассмотрим следующую переформулировку.

ГиПотезА 17'. Пусть $x$ - действительное число, $b \in \mathbb{N} u \mathrm{p}_{x_{b}}(n)<b^{n}$. Тогда х рационально или трансцендентно.

Известны следующие гораздо более слабые результаты.

ТеОрема 6.2.1 [86]. Пусть $x$-действителъное число. Если $x_{2}$ является последовательностью Штурма, то х трансцендентно.

В частности, например, двоичная запись $\sqrt{2}$ не является последовательностью Штурма. Впоследствии было получено более сильное утверждение.

Теорема 6.2.2 [87]. Пусть $x$ - действительное число, $b \in \mathbb{N} u \mathrm{p}_{x_{b}}(n)=$ $O(n)$. Тогда $x$ рачионально или трансцендентно. 
Отсюда сразу получается такое следствие.

СлеДСтвиЕ 6.2.3. Пусть $x$ - действительное число, $b \in \mathbb{N} u x_{b}$ - автоматная последовательность. Тогда $x$ рационально или трансцендентно.

ДоКАЗАТЕЛЬСтво. Следует из теоремы 6.1.5 и теоремы 6.2.2.

Мы же теперь можем получить и еще одно любопытное следствие.

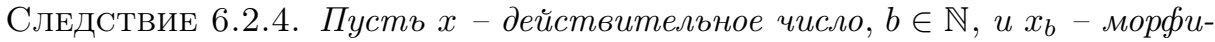
ческая почти периодическая последовательность. Тогда $x$ рационально или трансцендентно.

ДоКАЗАТЕЛЬСТвО. Следует из теоремы 6.1.4 и теоремы 6.2.2.

В [87] также формулируется следующая гипотеза.

ГиПотеЗА 18. Пусть $x$ - действительное число, $b \in \mathbb{N} u x_{b}$ - морфическая последовательность. Тогда $x$ рационально или трансцендентно.

Частные случаи этой гипотезы доказаны в [87], один из таких случаев следствие 6.2.3 (которое также является ответом на вопрос Кобхэма из [88]). Существенное продвижение в этой гипотезе (в частности, покрывающее результат следствия 6.2.4) получено в [89].

И наконец, самым сильным результатом в этой серии на настоящий момент является следующий.

Теорема 6.2.5 [90]. Пусть $x$ - действительное число, $b \in \mathbb{N} u \mathrm{p}_{x_{b}}(n)=$ $O\left(n(\log n)^{\theta}\right)$ для некоторого положительного действительного числа $\theta<1 / 11$. Тогда х рационально или трансцендентно.

Видно, что он еще по-прежнему очень далек от гипотезы Бореля.

6.3. Последовательности со сложными подсловами. Колмогоровская сложность конечного слова, неформально, - это количество информации в этом слове. Более формально, это длина кратчайшего бинарного описания этого слова, при таком способе описания, при котором слова получаются из своих коротких описаний применением универсального алгоритма. По-другому, колмогоровская сложность слова - это длина кратчайшей закодированной в бинарном алфавите программы без входа в каком-нибудь естественном языке программирования, печатающей это слово. Колмогоровская сложность определена с точностью до аддитивной константы (зависящей от языка программирования или конкретного универсального алгоритма, который берется в определении). Более детально с фундаментальным понятием колмогоровской сложности можно ознакомиться по [91] или [92] (см. также [93]).

Мы обозначаем через $K(u)$ сложность слова $u$. В этом пункте все конечные слова и бесконечные последовательности предполагаются бинарными. Одно из базовых свойств колмогоровской сложности, которое нам понадобится, следующее: для всех слов $u \in \mathbb{B}^{*}$ выполнено

$$
K(u)<|u|+C,
$$

поскольку каждое слово можно описать как минимум им самим. 
Хотя колмогоровская сложность измеряет количество информации в словах (и в других конечных объектах) и, следовательно, ее применение и связи с комбинаторикой слов представляются любопытными и естественными, о таких связях известно мало. Здесь можно отметить работы [14] (см. обсуждение дальше), [94], где найдено новое доказательство существования последовательностей с произвольным значением критической экспоненты (изначально результат получен в [95]) и, наконец, раздел 5 работы [96], расширенной версией которого и является настоящий пункт.

Основной целью данного пункта является обсуждение следующей гипотезы.

ГипотезА 19 (Ан. Мучник, 2005). Для любого $0<\alpha<1$ cуществует почти периодическая последовательность $x$, для которой одновременно выполнено следующее:

1) некоторая верхняя оценка на $\mathrm{R}_{x}$ вычислима;

2) неравенство $K(u)>\alpha|u|$ выполнено для любого подслова и последовательности $x$.

Гипотеза не доказана даже хотя бы для какого-нибудь фиксированного $\alpha$.

Свойства последовательности из этой гипотезы объединяют в себе до некоторой степени вычислимость (вычислимый регулятор почти периодичности) и до некоторой степени случайность (почти максимальная возможная колмогоровская сложность подслов). Однако эта последовательность не может быть ни по-настоящему вычислимой (это означало бы логарифмическую сложность всех начал), ни по-настоящему случайной в смысле Мартин-Лёфа (это означало бы, что в нее входят все возможные слова, в том числе простые).

Было получено много результатов, близких к гипотезе 19, например, следующее утверждение.

ТеОрема 6.3.1 [97]. Для любого $0<\alpha<1$ существует последовательность $x \in \mathbb{B}^{\mathbb{N}}$ такая, что неравенство $K(u)>\alpha|u|$ выполнено для всех подслов и последовательности $x$.

Так как по предложению 1.6.4 для каждой последовательности существует почти периодическая последовательность, все подслова которой являются подсловами исходной, в качестве следствия из теоремы 6.3.1 выводим существование почти периодической последовательности со сложными подсловами. Теперь задача в том, чтобы найти такую последовательность с вычислимой верхней оценкой на регулятор почти периодичности.

Можно вычислимо оценить сверху другую характеристику почти периодической последовательности. Пусть $x \in \mathscr{A} \mathscr{P}$. Определим $\mathrm{R}_{x}^{\prime}(n)$ как минимальное такое $l$, что слово $x[0, n-1]$ входит в любое подслово длины $l$ в $x$ (см. также [50]). Очевидно, $\mathrm{R}_{x} \geqslant \mathrm{R}_{x}^{\prime}$, поэтому для любого $x \in \mathscr{A} \mathscr{P}$ если какая-то верхняя оценка на $\mathrm{R}_{x}$ вычислима, то то же верно и про $\mathrm{R}_{x}^{\prime}$. По существу, следующее утверждение доказано в [14].

ТеОрема 6.3.2 [14]. Для любого $0<\alpha<1$ существует почти периодическая последовательность $x$, для которой одновременно выполнено следующее:

1) какая-то верхняя оценка на $\mathrm{R}_{x}^{\prime}$ вычислима;

2) неравенство $K(u)>\alpha|u|$ выполнено для любого подслова и последовательности $x$. 
При доказательстве теоремы 6.3.2 используется следующий простой метод назовем его арифметическим - построения почти периодических последовательностей. Пусть $n_{i}$ - возрастающая последовательность натуральных чисел такая, что $n_{i} \mid n_{i+1}$ для любого $i$. Возьмем произвольную последовательность $x$ и изменим в ней некоторые элементы так, чтобы она стала почти периодической. Первым шагом заменим все отрезки вида $x\left[i n_{1}, i n_{1}+n_{0}-1\right]$ для $i \geqslant 1$ на отрезок $x\left[0, n_{0}-1\right]$. Далее заменим все отрезки вида $x\left[i n_{2}, i n_{2}+n_{1}-1\right]$ для $i \geqslant 1$ на отрезок $x\left[0, n_{1}-1\right]$. И так далее: на $k$-м шаге процедуры мы заменяем все отрезки вида $x\left[i n_{k+1}, i n_{k+1}+n_{k}-1\right]$ для $i \geqslant 1$ на отрезок $x\left[0, n_{k}-1\right]$. За счет условия $n_{i} \mid n_{i+1}$ для любого $i$ все замены друг с другом согласованы: если какой-то символ на некотором шаге был заменен, то дальше он уже заменяться не будет. Отсюда следует, что последовательность, полученная такой процедурой, будет почти периодической: мы обеспечили достаточную встречаемость для каждого префикса. Более того, мы обеспечили каждому префиксу встречаемость по арифметической прогрессии, из чего следует, что каждая последовательность, полученная по вышеописанной процедуре, будет точно почти периодической. Однако, по всей видимости, так получаются не все точно почти периодические последовательности.

ГиПотЕзА 20. Существуют точно почти периодические последовательности, которые не порождаются арифметическим методом построения последовательностей.

Другое ослабление гипотезы Мучника может быть получено, если мы потребуем близости к случайности не от всех подслов последовательности, а только от всех префиксов. По существу, следующий результат получен в [46].

Теорема 6.3.3 [46]. Для любого $0<\alpha<1$ существует почти периодическая последовательность $x$, для которой одновременно выполнено следующее:

1) какая-то верхняя оценка на $\mathrm{R}_{x}$ вычислима;

2) неравенство $K(u)>\alpha|u|$ выполнено для любого префикса и последовательности $x$.

Техника, с помощью которой этот результат доказывается в работе [46], использует универсальный метод построения почти периодических последовательностей (см. п. 2.4). Это один из возможных способов пытаться доказать гипотезу 19. (Строго говоря, в работе [46] дана конструкция, использующая $\mathscr{A} \mathscr{P}$-схемы, которая не дает, вообе говоря, оценки на регулятор почти периодичности - см. гипотезу 5. Но эту конструкцию можно модифицировать, заменив использование $\mathscr{A} \mathscr{P}$-схем $\mathscr{G} \mathscr{A} \mathscr{P}$-схемами и тем самым обеспечив вычислимость оценки на регулятор.)

Естественное понятие подсловной сложности часто используется для характеризации конечных слов и бесконечных последовательностей. Следующие леммы описывают связи между колмогоровской и подсловной сложностью.

Лемма 6.3.4. Для произвольной $x \in \mathbb{B}^{\mathbb{N}}$ для любого $n$ существует такое $C$, что для любого $m \geqslant n$ для каждого $u \in \operatorname{Fac}_{m}(x)$ выполнено $K(u) \leqslant$ $\lceil m / n\rceil \log \mathrm{p}_{x}(n)+C$. 
ДокАЗАТЕЛЬСтво. Возьмем слово длины $m$, входящее в последовательность. Разделим его на блоки длины $n$ (возможно, последний блок будет неполной длины) - получится $\lceil m / n\rceil$ блоков. Каждый из получившихся блоков - слово длины $n$ (последнее, возможно, короче $n$ ), входящее в последовательность $x$. Всего в последовательность $x$ входит $\mathrm{p}_{x}(n)$ слов длины $n$, поэтому на задание каждого из блоков достаточно потратить $\log \mathrm{p}_{x}(n)$ битов. Кроме того, для задания длины последнего блока достаточно $\log \log \mathrm{p}_{x}(n)$ битов, что есть константа от $m$. Лемма доказана.

Лемма 6.3.5. Для произвольной $x \in \mathbb{B}^{\mathbb{N}}$ для любого $n$ существует $u \in$ $\operatorname{Fac}_{n}(x)$ такое, что $K(u) \geqslant \log \mathrm{p}_{x}(n)$.

ДокАзАтельство. Всего слов, имеющих сложность меньше $\log \mathrm{p}_{x}(n)$, не больше, чем $2^{\log \mathrm{p}_{x}(n)}-1=\mathrm{p}_{x}(n)-1$, поэтому хотя бы одно из слов множества $\operatorname{Fac}_{n}(x)$ имеет сложность не меньше, чем $\log \mathrm{p}_{x}(n)$.

Предел $\lim _{n \rightarrow \infty} n^{-1} \log \mathrm{p}_{x}(n)$ существует для любой последовательности $x$ и называется ее топологической энтропией (см. [98], [73]); мы обозначаем эту величину через $E_{t}(x)$. Это действительное число между 0 и 1 , некоторым образом описывающее, насколько детерминирована последовательность: чем ближе $E_{t}(x)$ к 0, тем более детерминирована последовательность $x$ и тем менее хаотична. Если пытаться определять аналогичную числовую характеристику в терминах колмогоровской сложности, то естественным выбором будет $E_{k}(x)=\lim _{n \rightarrow \infty} n^{-1} \max \left\{K(u): u \in \operatorname{Fac}_{n}(x)\right\}$. Из этого определения неясно даже, когда существует $E_{k}(x)$, но из лемм 6.3.4 и 6.3.5 можно вывести следующее.

ТЕОРема 6.3.6. Для любой последовательности $x$ число $E_{k}(x)$ существует и равно $E_{t}(x)$.

ДокАЗАТЕЛЬСтво. Из леммы 6.3 .5 сразу следует, что

$$
\frac{1}{n} \max \left\{K(u): u \in \operatorname{Fac}_{n}(x)\right\} \geqslant \frac{1}{n} \log \mathrm{p}_{x}(n),
$$

откуда

$$
\liminf _{n \rightarrow \infty} \frac{1}{n} \max \left\{K(u): u \in \operatorname{Fac}_{n}(x)\right\} \geqslant E_{t}(x) .
$$

Теперь зафиксируем $n$. Тогда для некоторой константы $C$ для любого $m \geqslant n$ для любого $u \in \operatorname{Fac}_{m}(x)$ имеем $K(u) \leqslant\lceil m / n\rceil \log \mathrm{p}_{x}(n)+C$. Отсюда

$$
\frac{1}{m} \max \left\{K(u): u \in \operatorname{Fac}_{m}(x)\right\} \leqslant \frac{1}{m}\left\lceil\frac{m}{n}\right\rceil \log \mathrm{p}_{x}(n)+\frac{C}{m} .
$$

Следовательно,

$$
\limsup _{m \rightarrow \infty} \frac{1}{m} \max \left\{K(u): u \in \operatorname{Fac}_{m}(x)\right\} \leqslant \frac{1}{n} \log \mathrm{p}_{x}(n)
$$

для любого $n$, откуда следует, что

$$
\limsup _{m \rightarrow \infty} \frac{1}{m} \max \left\{K(u): u \in \mathrm{Fac}_{m}(x)\right\} \leqslant E_{t}(x) .
$$


Отсюда получаем, что

$$
\begin{aligned}
E_{t}(x) & \leqslant \liminf _{n \rightarrow \infty} \frac{1}{n} \max \left\{K(u): u \in \operatorname{Fac}_{n}(x)\right\} \\
& \leqslant \limsup _{n \rightarrow \infty} \frac{1}{n} \max \left\{K(u): u \in \operatorname{Fac}_{n}(x)\right\} \leqslant E_{t}(x) .
\end{aligned}
$$

Следовательно, $E_{k}(x)=E_{t}(x)$.

СлЕДСтвИЕ 6.3.7. Для любого $0<\alpha<1$ cyществует почти периодическая последовательность $x$ с вычислимой оченкой сверху на регулятор почти периодичности такая, что $E_{t}(x)>\alpha$.

ДокАЗАТЕЛьство. Действительно, последовательность из теоремы 6.3 .3 подходит, что следует из теоремы 6.3.6.

Однако гипотеза Мучника до сих пор остается открытой.

6.4. Мера апериодичности. Периодические последовательности имеют самую простую структуру среди всех последовательностей над конечным алфавитом, поэтому естественно было бы научиться измерять, насколько данная последовательность далека от любой периодической. В [99] вводится понятие меры апериодичности последовательности. Неформально, мера апериодичности последовательности - это максимальное такое число $\alpha$, что последовательность с любым своим нетривиальным сдвигом имеет хотя бы долю $\alpha$ различий.

Формальное определение этого понятия основано на дискретном аналоге расстояния Безиковича $d_{B}$, использованном в [5] для определения почти периодических по Безиковичу последовательностей, см. с. 32. Тот же подход был использован в [100] для определения $\alpha$-апериодических последовательностей. По существу, в [100] было замечено, в нашей терминологии, что если $\operatorname{AM}(x)>\alpha$ для последовательности $x$, то $x$ находится на расстоянии хотя бы $\alpha / 2$ от любой заключительно периодической последовательности, в смысле расстояния $d_{B}$.

Напомним (см. раздел 1), что расстояние Безиковича определяется как

$$
d_{B}(x, y)=\liminf _{n \rightarrow \infty} \frac{1}{n} \#\{i: 0 \leqslant i \leqslant n-1, x(i) \neq y(i)\} .
$$

Определим меру апериодичности

$$
\operatorname{AM}(x)=\inf \left\{d_{B}\left(x, L^{n} x\right): n \geqslant 1\right\},
$$

где $L$ обозначает операцию левого сдвига. Другими словами, $\operatorname{AM}(x)-$ это максимальное число от 0 до 1 такое, что можно утверждать, что у последовательности $x$ с любым ее собственным сдвигом хотя бы доля $\operatorname{AM}(x)$ символов различны.

Одним из мотивов для изучения понятия меры апериодичности стала следующая гипотеза Б. Дюрана, А. Ромащенко и А. Шеня: Для любого $\alpha<1$ существует автоматная последовательность $x$ такая, что $\operatorname{AM}(x) \geqslant \alpha$. Эта гипотеза доказана в [99], см. теорему 6.4.6. Теорема 6.4.6 позволяет упростить конструкцию сильно апериодического замощения из [100].

Кроме того, понятие меры апериодичности достаточно естественно и просто, чтобы представлять самостоятельный интерес. 
Ясно, что если последовательность $x$ заключительно периодическая с периодом $p$, то $d_{B}\left(x, L^{p} x\right)=0$ и, следовательно, $\mathrm{AM}(x)=0$. Обратное не всегда верно - несложно привести пример апериодической последовательности, мера апериодичности которой равна 0. Тем не менее, естественно считать, что чем меньше число $\mathrm{AM}(x)$, тем ближе $x$ к периодической последовательности. Одним из подтверждений является следующий результат.

Теорема 6.4.1 [99]. Если $x$ - последователъность Штурма, то $\operatorname{AM}(x)=0$.

Если в вышеупомянутой гипотезе не требовать автоматности последовательности, то утверждение становится почти очевидным.

ТЕОРема 6.4 .2 [99]. Пусть $x$ - случайная последовательность в алфавите из $k$ символов. Тогда $\operatorname{AM}(x)=1 / k$.

Для фиксированного алфавита можно дать и верхнюю оценку на меру апериодичности.

ТеОрема 6.4 .3 [99]. Если последовательность $x$ состоит из не более чем $k$ символов, то $\operatorname{AM}(x) \leqslant 1-1 /(2 k)$.

Отметим, что в [101] была получена более сильная верхняя оценка $1-1 / k$ на меру апериодичности последовательности из не более чем $k$ символов.

Интересно также посчитать меру апериодичности некоторых конкретных последовательностей.

TeOpema 6.4.4 [99]. $\operatorname{AM}(\mathbf{t})=\frac{1}{3}$.

Заметим, что по существу в [5] было доказано $\mathrm{AM}(\mathbf{t}) \geqslant 1 / 4$.

Изначально предполагалось, что обобщение последовательности Туэ-Морса - последовательности Пруэ $\mathbf{t}_{k}$ (см. п. 5.1) - могут иметь высокую меру апериодичности. Оказывается, это не так.

Teоpema 6.4.5 [99]. $\operatorname{AM}\left(\mathbf{t}_{k}\right) \leqslant \frac{2}{k+1}-\frac{2}{k^{k-1}(k+1)}$.

Теперь приведем конструкцию автоматной последовательности с мерой апериодичности, сколь угодно близкой к 1 .

Пусть $k \geqslant 3$ и $\varphi:\{0, \ldots, k-1\}^{*} \rightarrow\{0, \ldots, k-1\}^{*}-$ морфизм такой, что

$$
(\varphi(i))(j)=i+1+2+\cdots+(j-1)+j \quad \text { для } 0 \leqslant i, j \leqslant k-1,
$$

где + всегда берется по модулю $k$, а $u(i)$ обозначает $i$-й символ конечного слова $u$. Положим $x_{k}=\varphi^{\infty}(0)$. Например, если $k=5$, то $\varphi$ получается таким:

$$
\begin{aligned}
& \varphi(0)=01310, \\
& \varphi(1)=12421, \\
& \varphi(2)=23032, \\
& \varphi(3)=34143, \\
& \varphi(4)=40204,
\end{aligned}
$$

и $x_{5}=013101242134143124210131012421 \ldots$.

Можно доказать (см. [99]), что если $k$ простое, то $\operatorname{AM}\left(x_{k}\right)=1-2 / k$. Таким образом, получаем следующий результат. 
Теорема 6.4.6 [99]. Для любого $\alpha<1$ существует автоматная последовательность $x$ такая, что $\operatorname{AM}(x) \geqslant \alpha$.

В заключение приведем некоторые открытые вопросы о мере апериодичности бесконечных последовательностей (подробнее см. [99]).

ПроБлемА 21. 1) Для каждого $k \geqslant 2$ каково максимальное значение $\operatorname{AM}(x)$ для автоматных последовательностей $x$ в алфавите из $k$ символов?

2 ) Для каждого $k \geqslant 2$ каково максимальное значение $\operatorname{AM}(x)$ для $k$-автоматных последовательностей $x$ ?

ПроБЛЕмА 22. Посчитайте меру апериодичности других известных последовательностей и классов последовательностей, например, последовательностей Тёплица (см. п. 7.3), каких-нибудь автоматных и морфических последовательностей (см. пп. 5.1 и 5.2), каких-нибудь обобщений последовательностей Штурма (см. п. 1.2) и т. д.

\section{5. Предельное поведение регулятора почти периодичности.}

Сложность почти периодической последовательности можно понимать и как сложность устройства ее регулятора почти периодичности. Следующий результат стал положительным ответом на долгое время остававшийся открытым вопрос Морса и Хедлунда, сформулированный в [5].

ТЕОрема 6.5.1 [102]. Если последовательность $x$ апериодическая, то не существует конечного предела последовательности $\mathrm{R}_{x}(n) / n$ nри $n \rightarrow \infty$.

Удобно кроме регулятора почти периодичности последовательности определить также функцию $\mathrm{r}_{x}(n)=\mathrm{R}_{x}(n)-n+1$, которая равна максимальному расстоянию между соседними вхождениями слова $u$ длины $n$ (расстояние берется между левыми концами вхождений, а максимум берется по всем словам длины $n$ и по всем расстояниям). Следуя [50], определим коэфбиииент почmи периодичности $\rho_{x}$ последовательности $x$ как $\rho_{x}=\limsup _{n \rightarrow \infty}\left(\mathrm{r}_{x}(n) / n\right)$. Несложно видеть, что для периодической последовательности $x$ коэффициент почти периодичности равен 1 . Кроме того, последовательность линейно почти периодическая (см. с. 43) тогда и только тогда, когда ее коэффициент почти периодичности конечен.

В [5] поставлен вопрос нахождения наилучшей нижней оценки на $\mathrm{r}_{x}$ для непериодических $x$. Из предложения 6.1.1 несложно видеть, что $\rho_{x} \geqslant 2$ для непериодических $x$. В [50] доказано, что $\rho_{x} \geqslant 3$ для непериодических $x$. Рози и Кассэнь выдвинули следующую гипотезу.

ГиПотезА 23 [50], [103]. Для любой непериодической последователъности $x$ выполнено $\rho_{x} \geqslant(5+\sqrt{5}) / 2=3.618 \ldots$.

Поскольку для последовательности Фибоначчи $\rho_{\mathbf{f}}=(5+\sqrt{5}) / 2$ (см. [50]), доказательство этой гипотезы стало бы полным ответом на упомянутый вопрос из [5].

Основным средством в доказательстве теоремы 6.5.1, а также в предполагаемом уже существующем, но пока не опубликованном доказательстве гипотезы 23 (см. комментарии в [50]) являются графы Рози последовательностей, широко используемые в комбинаторике слов (определение и некоторые свойства можно найти, например, в [102]). 


\section{7. Близость к периодичности: альтернативные подходы}

В этом разделе мы говорим о несколько менее известных и менее изученных, но также интересных вариантах понятий, обобщающих понятие периодической последовательности.

7.1. Квазипериодические последовательности. В [104], [105] была предложена следующая идея обобщения понятия периодичности. Будем говорить, что конечное слово $w$ является квазипериодом $x$ (конечного слова или бесконечной последовательности), если каждую позицию в $x$ можно покрыть вхождением $w$ в $x$. Более формально, для каждого $i: 0 \leqslant i \leqslant|x|-1$ найдутся $k, l: k \leqslant i \leqslant l$ такие, что $x[k, l]=w$ (для бесконечного $x$ можно положить $|x|=\infty)$.

Конечное слово квазипериодично, если у него есть хотя бы один квазипериод, отличный от него самого; в противном случае слово называют минимальным (или в [106] суперпримитивным). У каждого конечного слова есть ровно один минимальный квазипериод (т. е. квазипериод, являющийся минимальным словом), см. [106].

Последовательность называется квазипериодической, если у нее существует хотя бы один квазипериод. Класс всех квазипериодических последовательностей обозначим $\mathscr{Q} \mathscr{P}$. Существуют последовательности с бесконечным количеством минимальных квазипериодов (например, последовательность Фибоначчи, см. [106]). Последовательности с бесконечным количеством различных квазипериодов назовем мульти-квазипериодическими (multi-scale quasiperiodic, см. [107]). Класс мульти-квазипериодических последовательностей обозначим $\mathscr{M} \mathscr{Q} \mathscr{P}$. Ясно, что существуют последовательности вообще без квазипериодов. Как показано в [106], любая промежуточная ситуация реализуется: для каждого $n$ можно построить последовательность, у которой ровно $n$ минимальных квазипериодов.

Определение квазипериодичности, несмотря на свою естественность, было впервые рассмотрено относительно недавно. В [104] появилось определение для конечных слов, которое затем было расширено на последовательности в [105]. Там же поставлены различные открытые вопросы. В [106] авторы получают ответы на некоторые из них. В [108] они получают полное описание квазипериодических последовательностей Штурма. В работе [107] рассмотрено понятие мульти-квазипериодичности.

Оказывается, класс $\mathscr{Q} \mathscr{P}$ находится в общем положении с классом $\mathscr{A} \mathscr{P}$. Неравенство $\mathscr{Q} \mathscr{P} \backslash \mathscr{A} \mathscr{P} \neq \varnothing$ очевидно. Действительно, например, в $\mathscr{Q} \mathscr{P}$ лежит произвольная последовательность из 0 и 1, начинающаяся с 1 и не содержащая 00 и 111 (с квазипериодом 101), и, очевидно, среди таких последовательностей есть не почти периодические. Неравенство $\mathscr{A} \mathscr{P} \backslash \mathscr{Q} \mathscr{P} \neq \varnothing$ следует из результатов [106], где авторы приводят примеры последовательностей Штурма, не являющихся квазипериодическими (в ответ на вопрос из [105]).

Таким образом, квазипериодичность и почти периодичность - разные подходы к обобщению периодичности, что является дополнительным стимулом к изучению недавно появившегося класса квазипериодических последовательностей. Ясно, однако, что имеет место включение $\mathscr{M} \mathscr{Q} \mathscr{P} \subseteq \mathscr{A} \mathscr{P}$, а также 
невырожденность $\mathscr{P} \subsetneq \mathscr{M} \mathscr{Q} \mathscr{P}$ (см. [107]; из результатов оттуда следует также, что класс $\mathscr{M} \mathscr{Q} \mathscr{P}$ континуален). Таким образом, некоторая связь между почти периодичностью и квазипериодичностью (правда, уже уточненной) все же есть. Неравенство $\mathscr{M} \mathscr{Q} \mathscr{P} \subsetneq \mathscr{A} \mathscr{P}$ сразу следует из характеризации подсловной сложности произвольного слова $x \in \mathscr{M} \mathscr{Q} \mathscr{P}$ :

$$
\liminf _{n \rightarrow \infty} \frac{\mathrm{p}_{x}(n)}{n^{2}} \leqslant 1
$$

(cм. [107]).

Как показано в [107], подсловная сложность квазипериодических последовательностей асимптотически может быть почти произвольной, с точностью до эквивалентности специального вида. В [106] приведен несложный пример последовательности, у которой подсловная сложность экспоненциальна, с основанием экспоненты примерно 1.32. Остается открытым следующий вопрос.

ПроБлЕмА 24. Какая максимальная возможная подсловная сложность может быть у квазипериодической последовательности?

Про некоторые вопросы из [105] авторы [106] пишут, что они нуждаются в более точной формулировке, поскольку в них идет речь о последовательностях, в которых все факторы квазипериодичны, но ясно, что слово длины 1 никогда не квазипериодично. Один из них, вопрос 6 (существует ли неквазипериодическая последовательность, у которой все факторы квазипериодичны?), переформулируется так: можно ли найти не квазипериодическую последовательность, у которой найдется бесконечное количество квазипериодических префиксов? В [106] на него дается положительный ответ.

В контексте настоящей статьи естественно задать следующий вопрос.

ПроБлЕмА 25. 1) Верно ли, что класс квазипериодических последовательностей замкнут относительно конечно-автоматных преобразований?

2) Если ответ на предыдущий вопрос положительный, то можно ли это доказать в каком-нибудь смысле эффективно и получить критерий разрешимости монадической теории квазипериодической последовательности (см. раздел 3)?

7.2. Разреженные периодичности. В [109] предложен любопытный подход к обобщению понятия периодичности, сходный с тем, который изложен в п. 7.1.

Назовем шаблоном слово в алфавите $A \cup\{\square\}$, где $\square \notin A$ означает для нас пробел, пропуск, или прозрачное окно. Конечное слово $u \in A^{*}$ назовем разреженно периодическим, если найдется такой шаблон $v$, что его копиями можно покрыть все слово $u$, причем каждый символ покрыт ровно один раз; при этом мы считаем, что при наложении шаблона на слово окно $\square$ не покрывает находящийся под ним символ - этот символ "просвечивает" из-под окна. Например, слово 0011 можно покрыть шаблоном $0 \square 1$.

В отличие от обычной периодичности, бывают слова с несколькими несравнимыми разреженными периодами (если сравнивать по вложенности) - в [109] приводится кратчайший известный пример такого слова из 24 символов в алфавите из двух символов: слово 
имеет минимальные периоды

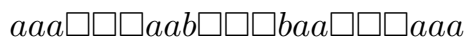

и

$a a a a \square \square \square \square b a a b \square \square \square \square a a a a$,

которые удобно увидеть, рассмотрев две разметки этого слова:

$$
\text { aaa }|a a a| a a b|a a b| b a a|b a a| a a a \mid a a a
$$

И

aaaa $|a a a a| b a a b|b a a b| a a a a \mid a a a a$.

Однако можно найти связь между обычным периодом и разреженным: минимальный разреженный период слова является также минимальным разреженным периодом его минимального настоящего периода. Кроме того, представлена классификация всех разреженных периодов произвольного слова и получена рекуррентная формула для максимального количества разреженных периодов для слов длины $n$. Наконец, получен алгоритм нахождения всех минимальных разреженных периодов слова длины $n$, время работы $O(n \log n \log \log n)$.

В конце работы [109] приведено несколько открытых вопросов. По мнению авторов, наиболее важным является следующий.

ПроБЛЕмА 26 [109]. Предложить естественный способ обобщения понятия "чистой" (pure) разреженной периодичности (по аналогии с pure и ultimate (заключительной) периодичностью). Это может помочь описать регулярности в большем количестве слов и получить новые алгоритмы компрессии.

Для нас наиболее существенным является следующее замечание негативного характера: при прямом обобщении понятия разреженной периодичности на бесконечные последовательности получаются только периодические последовательности, как следует, например, из теоремы 2 работы [110].

Таким образом, остается следующая проблема.

ПроБлЕмА 27. Предложить нетривиальный способ обобщения понятия разреженной периодичности на бесконечные последовательности, который позволит получить для них критерий разрешимости монадической теории или теории первого порядка.

7.3. Последовательности Тёплица, периодически альтернируемые морфизмы и последовательность Колакоски. В работе [111] Тёплиц ввел конструкцию для определения почти периодических функций на действительной прямой. В работе [112] эта конструкция модифицируется для определения бесконечных слов и эти слова называются словами Тёплица (мы для единообразия будем говорить о последовательностях). Краткий нижеследующий обзор о последовательностях Тёплица сделан в основном по работе [113].

Пусть шаблон (как и в п. 7.2) - слово в алфавите $A \cup\{\square\}$, где $\square \notin A$ означает для нас пробел или пропуск. Из шаблона $w$ можно получить последовательность следующим образом. Пусть $T_{w}^{0}=w^{\infty}$ - периодическая последовательность в алфавите $A \cup\{\square\}$ с периодом $w$. Для каждого $i$ последовательность $T_{w}^{i+1}$ получается из $T_{w}^{i}$, если все пробелы $\square$ последовательно заполнять 
символами последовательности $T_{w}^{0}=w^{\infty}$ : если идти слева направо, в самое левое вхождение поместить нулевой символ последовательности $T_{w}^{0}$, в следующий встреченный - первый символ последовательности $T_{w}^{0}$, и т. д. Последовательность $T_{w}=\lim _{i \rightarrow \infty} T_{w}^{i}$ в алфавите $A$ называется последовательностью Тёnлица, полученной из шаблона $w$. Если $|w|=p$ и $|w|_{\square}=q$, то последовательность $T_{w}$ называется последовательностью Тёплица типа $(p, q)$.

Например, пусть $w=1 \square 0 \square$. Тогда

$$
\begin{aligned}
& T_{w}^{0}=1 \square 0 \square 1 \square 0 \square 1 \square 0 \square 1 \square 0 \square 1 \square 0 \square 1 \square 0 \square 1 \square 0 \square 1 \square 0 \square \ldots, \\
& T_{w}^{1}=110 \square 100 \square 110 \square 100 \square 110 \square 100 \square 110 \square 100 \square \ldots, \\
& T_{w}^{2}=1101100 \square 1100100 \square 1101100 \square 1100100 \square \ldots, \\
& T_{w}^{3}=110110011100100 \square 110110001100100 \square \ldots, \\
& T_{w}^{4}=1101100111001001110110001100100 \square \ldots, \\
& T_{w}^{5}=11011001110010011101100011001001 \ldots,
\end{aligned}
$$

т. е. $T_{w}=11011001110010011101100011001001 \ldots$. Это известная "последовательность сгибания бумаги" (paperfolding sequence), например, см. [42]. (Эта последовательность получается следующим образом: возьмем ("одномерную") полоску бумаги и согнем ее посередине; потом получившееся опять согнем посередине, и т. д. Каждый раз необходимо сгибать в одном и том же направлении (например, положив полоску на стол и все время сгибая левой рукой вправо). После сгибания некоторое количество раз, разогнув полоску, мы получим начальный отрезок последовательности сгибания бумаги: 1 соответствует "горке" на полоске, а 0 соответствует "ямке".)

Как несложно видеть, любая последовательность Тёплица почти периодична. В [113] получен критерий периодичности для последовательностей Тёплица. Кроме того, в [113] дана классификация последовательностей Тёплица, для формулировки которой нам понадобится новое понятие - обобщение понятия морфических последовательностей - введенное в работе [114].

Пусть $H=\left\{h_{0}, h_{1}, \ldots, h_{p-1}\right\}$ - произвольное множество нестирающих морфизмов $A^{*} \rightarrow A^{*}$. Определим отображение $H$ на слове $a_{0} a_{1} \ldots a_{n} \in A^{*}$ как

$$
H\left(a_{0} a_{1} \ldots a_{n}\right)=\alpha_{0} \alpha_{1} \ldots \alpha_{n}, \quad \text { где } \alpha_{i}=h_{k}\left(a_{i}\right), k \equiv i(\bmod p) .
$$

Если для какой-то буквы $s$ слово $H(s)$ начинается с $s$, то можно корректно определить последовательность $H^{\infty}(s)=\lim _{n \rightarrow \infty} H^{n}(s)$. Будем говорить, что эта последовательность порождается периодически альтернируемыми морфизмами $h_{0}, h_{1}, \ldots, h_{p-1}$.

Ясно, что все чисто морфические последовательности порождаются периодически альтернируемыми морфизмами, точнее, одним морфизмом. Менее тривиальный пример - последовательность Колакоски - рассмотрен ниже.

В [115] доказано, что для любого полинома существуют последовательности, порождаемые периодически альтернируемыми морфизмами, с подсловной сложностью не менее этого полинома. В частности, такие последовательности не все морфические, поскольку (см. п. 5.2) подсловная сложность морфической последовательности не более чем квадратичная. Неизвестно, может ли 
подсловная сложность последовательностей, порожденных периодически альтернируемыми морфизмами, быть экспоненциальной.

ПроБлема 28. Разрешима ли (монадическая или первого порядка) теория последовательностей, порождаемых периодически альтернируемыми морфизмами?

Напомним, что монадическая теория морфической последовательности всегда разрешима (теорема 5.2.2). Можно надеяться, что и в случае периодически альтернируемых морфизмов это так. По аналогии с планом доказательства разрешимости монадических теорий, описанным в разделе 3 , можно детализировать проблему 28.

ПроБЛЕмА 29. 1) Верно ли, что класс последовательностей, порожденных периодически альтернируемыми морфизмами, сохраняется при конечно-автоматных преобразованиях?

2) Если ответ на предыдущий вопрос положительный, можно ли доказать эффективный вариант этого утверждения?

3) Существует ли алгоритм проверки по описанию последовательности, порожденной периодически альтернируемыми морфизмами, того, входит ли в нее данный символ бесконечное количество раз?

Теорема 7.3.1 [113]. 1) Последовательности Тёллица типа $(p, 1)$ являются чисто морфическими.

2) Последовательности Тёллица типа $(t q, q)$ являются морфическими.

3) Произвольные последовательности Тёплича порождаются периодически альтернируемыми морфизмами.

Естественным представляется, особенно в виду теоремы 5.2 .2 и проблемы 28, сформулировать следующую гипотезу.

ГипотезА 30. Монадическая теория любой последовательности Тёплица разрешима.

Скорее всего, эту гипотезу можно доказать, доказав, что множество подслов любой последовательности Тёплица разрешимо (последовательность Тёплица, очевидно, вычислима, и тогда можно воспользоваться следствием 3.1.6). Более того, достаточно доказать, что функция подсловной сложности вычислима. По всей видимости, это можно сделать, проанализировав доказательство результата из [113], в котором найдена асимптотика подсловной сложности последовательностей Тёплица. При этом отметим, что из описания асимптотики функции подсловной сложности, вообе говоря, прямо не следует вычислимость функции подсловной сложности.

Последовательность Колакоски - одна из самых загадочных последовательностей в комбинаторике слов, про нее есть гораздо больше открытых вопросов, чем известных фактов.

Определяется последовательность Колакоски так. Это бесконечная вправо последовательность, состоящая из 1 и 2, причем если записать длины блоков из подряд идущих одинаковых символов, то получится сама эта последовательность. Последовательность Колакоски начинается так:

$$
\mathbf{k}=22112122122112112212112 \ldots
$$


Сначала идет блок из 2 двоек, потом 2 единицы, 1 двойка, 1 единица, 2 двойки и т. д. Можно начать записывать последовательность Колакоски и с 1, руководствуясь тем же самым определением, но тогда получится в точности последовательность $1 \mathbf{k}$.

Последовательность k впервые появилась в заметке Колакоски [116]. В [117] он доказывает, что эта последовательность не периодическая. См. также [118].

Главная гипотеза про последовательность $\mathbf{k}$ заключается в следующем.

ГиПотезА 31. Частота символа 1 (а значит, и символа 2) в последовательности $\mathbf{k}$ равна $1 / 2$.

До сих пор неизвестно, верно ли это. Более того, неизвестно, существует ли вообще частота 1 , но известно, что если она существует, то обязательно равна $1 / 2$. Компьютерные эксперименты подтверждают гипотезу о частоте $1 / 2$. Более того, если $o_{n}$ - количество единиц в начальном отрезке $\mathbf{k}$ длины $n$, то компьютерные эксперименты позволяют предположить, что $o_{n}=0.5 n+O(\log n)$ (см. [119]), в то время как для случайной последовательности можно было бы утверждать лишь, что $o_{n}=0.5 n+O(\sqrt{n})$.

ПроБлемА 32. 1) Верно ли, что если какое-то слово входит в $\mathbf{k}$ (например, 22112), то оно входит еще хотя бы раз?

2) Верно ли, что если какое-то слово входит в $\mathbf{k}$, то входит и слово, полученное из этого заменой 1 на 2 и 2 на 1 ?

3) Верно ли, что если какое-то слово входит в $\mathbf{k}$, то входит и слово, полученное из этого, если его записать задом наперед?

Ответы на все эти вопросы неизвестны (см. [120], [121]). В частности, неизвестно, является ли $\mathbf{k}$ рекуррентной или почти периодической. Однако известно, что из рекуррентности $\mathbf{k}$ следовало бы существование частоты 1 в ней. Неизвестна и подсловная сложность последовательности Колакоски.

K этой серии открытых и, по всей видимости, довольно сложных проблем мы добавим такую.

ПроблемА 33. Разрешима ли (монадическая или первого порядка) теория последовательности $\mathbf{k}$ ?

Формально из разрешимости монадической теории последовательности Колакоски не следует ответ на сложные открытые вопросы, приведенные выше. Это может являться аргументом за то, что проблема 33 проще. Однако если доказать, что монадическая теория последовательности $\mathbf{k}$ разрешима, это дало бы возможность для дополнительных экспериментов. В частности, из этого следовало бы существование алгоритма, который по каждому слову проверяет, входит ли оно в $\mathbf{k}$, входит ли оно в $\mathbf{k}$ бесконечно много раз и т. п.

Тем не менее кое-что про последовательность Колакоски известно. Как уже было отмечено выше, она не периодическая. Кроме того, она не содержит кубов, а квадраты, которые в нее входят, имеют длину 2, 4, 6, 18 или 54 (см. [122]). Кроме того, последовательность Колакоски не является чисто морфической [123]. Является ли она морфической, неизвестно.

Последовательность Колакоски порождается периодически альтернируемыми морфизмами. Пусть $h_{0}(1)=2, h_{0}(2)=22, h_{1}(1)=1, h_{1}(2)=11$. Будем 
итерировать соответствующее отображение $H$ на символе 2 :

$$
\begin{aligned}
H(2) & =h_{0}(2)=22, \\
H(22) & =h_{0}(2) h_{1}(2)=2211 \\
H(2211) & =h_{0}(2) h_{1}(2) h_{0}(1) h_{1}(1)=221121, \\
H(221121) & =h_{0}(2) h_{1}(2) h_{0}(1) h_{1}(1) h_{0}(2) h_{1}(1)=221121221, \\
H(221121221) & =h_{0}(2) h_{1}(2) h_{0}(1) h_{1}(1) h_{0}(2) h_{1}(1) h_{0}(2) h_{1}(2) h_{0}(1) \\
& =22112122122112 \ldots
\end{aligned}
$$

Можно доказать, что $H^{\infty}(2)=\mathbf{k}[123]$.

Указатель терминов. В этом разделе собраны в виде таблицы ссылки на основные используемые в статье термины и понятия. Для каждого понятия дается соответствующее обозначение (если есть) и номер страницы, на которой появляется определение понятия.

\section{Понятие}

$\mathscr{A} \mathscr{P}$-порожденная $\mathscr{A} \mathscr{P}$-схемой последовательность

$\mathscr{A} \mathscr{P}$-порожденная $\mathscr{G} \mathscr{A} \mathscr{P}$-схемой последовательность

$\mathscr{A} \mathscr{P}$-схема

$\mathscr{G} \mathscr{A} \mathscr{P}$-порожденная $\mathscr{G} \mathscr{A} \mathscr{P}$-схемой последовательность

$\mathscr{G} \mathscr{A} \mathscr{P}$-схема

$k$-равномерный морфизм

автомат Бюхи

автомат Бюхи принимает последовательность

автомат Мюллера

автомат Мюллера принимает последовательность

автоматная последовательность

алгебраическое множество

алгебраическое число

алфавит

апериодическая последовательность

бесквадратная последовательность

бескванторная теория

бесконечное слово

бескубная последовательность

блочное произведение

буква

вращательные последовательности

вхождение слова в последовательность

выразимое свойство

вычислимое действительное число

вычислимый оператор на последовательностях
Обозначение Страница 
детерминированный автомат Бюхи 56

детерминированный автомат Мюллера 56

заключительно линейно почти периодическая последо- 44

вательность

заключительно периодическая последовательность $\quad \mathscr{E} \mathscr{P}$

заключительно почти периодическая последователь- $\quad \mathscr{E} \mathscr{A} \mathscr{P}$

ность

заключительно рекуррентная последовательность

$\mathscr{E} \mathscr{R} \quad 33$

канторовское расстояние

$d_{C}(x, y)$

квадрат слова

квазипериодические последовательности

$\mathscr{Q} \mathscr{P} \quad 81$

кодирование

колмогоровская сложность

конечно-автоматный преобразователь

коэффициент почти периодичности

куб слова

линейно почти периодическая последовательность

нестирающий морфизм

нормальное число

макросостояние

мера апериодичности

механическая последовательность

$\operatorname{AM}(x) \quad 78$

минимальная последовательность

$s_{\alpha, \rho}$

минимальный префикс

морфическая последовательность

морфизм

монадическая теория последовательности

мульти-квазипериодические последовательности

$\operatorname{MT}\langle\mathbb{N},<, x\rangle \quad 54$

$\mathscr{M} \mathscr{Q} \mathscr{P} \quad 81$

образ последовательности под действием преобразова-

теля

обратимый конечно-автоматный преобразователь

обобщенно почти периодическая последовательность

$\mathscr{G} \mathscr{A} \mathscr{P} \quad 33$

операция левого сдвига

L $\quad 35$

период

периодическая последовательность

$\mathscr{P} \quad 32$

периодически альтернируемые морфизмы

правильно $\mathscr{G} \mathscr{A} \mathscr{P}$-порожденная последовательность

предельное макросостояние $\quad 56$

предпериод

преобразователь подходит ко вхождению слова в состоянии $q$

префикс 
продолжаемый на букве морфизм

проекция

произведение последовательностей

$x \times y$

почти обратимый конечно-автоматный преобразователь

почти периодическая относительно меры близости функ-

ция

почти периодическая по Безиковичу последовательность

почти периодическая по Безиковичу функция

почти периодическая по Бору функция

почти периодическая последовательность

подсловная сложность

$\begin{array}{ll}\mathscr{A} \mathscr{P} & 32 \\ \mathrm{p}_{x}(n) & 70\end{array}$

подслово

полуалгебраическое множество

последовательности Какутани

последовательности Тёплица

последовательности Штурма

последовательность Колакоски

последовательности Пруэ

последовательность сгибания бумаги

последовательность Туэ-Морса

последовательность Фибоначчи

равномерно рекуррентная последовательность

равномерно чезаровская последовательность

равномерный конечно-автоматный преобразователь

разреженная периодичность

разрешимая теория

расстояние Безиковича

регулярный язык

регулятор почти периодичности

счетчиковый конечно-автоматный преобразователь

сбалансированное слово или последовательность

символ

слово

суффикс 
топологическая динамическая система

топологическая энтропия

точно почти периодическая последовательность

фактор

ход преобразователя

циклический конечно-автоматный преобразователь 51

чезаровская последовательность 46

чезаровский блок 46

чисто морфическая последовательность $\quad 67$

эффективно обобщенно почти периодическая последова- 33

тельность

эффективно почти периодическая последовательность 34

язык

Авторы благодарны С. Августиновичу, И. Богданову, Н. Верещагину, Р. Девятову, М. Раскину, А. Румянцеву, Ю. Уляшкиной, А. Фрид, А. Шеню, а также B. Adamczewski, J. Cassaigne, V. Diekert, B. Durand, J. Karhumäki, O. Kupferman, F. Nicolas, N. Rampersad, K. Saari за многочисленные полезные обсуждения на темы, затронутые в настоящей обзорной статье. Темы и результаты этой статьи неоднократно обсуждались на Колмогоровском семинаре МГУ, а также на семинаре “Алгоритмические вопросы алгебры и логики" под руководством академика С. И. Адяна, авторы благодарны всем участникам за внимание.

Работа над текстом продолжалась и когда один из его авторов - Андрей Альбертович Мучник (24.02.1958-18.03.2007) - скончался. Его вклад в деятельность двух других соавторов далеко не ограничивается формальными публикациями, перечисленными в настоящем обзоре и некрологе [124]. Завершение нашей работы над текстом обзора является для нас посильным вкладом в память об Андрее и продолжение его работ и идей в мировой математике. А. Л. Семенов, Ю.Л. Притыкин.

\section{Список литературы}

[1] J. S. Hadamard, "Les surfaces à courbures opposées et leurs lignes géodésiques", $J$. de Math. (5), 4 (1898), 27-73.

[2] G. D. Birkhoff, "Nouvelles recherches sur les systèmes dynamiques", Memoriae Pont. Acad. Scient. Novi Lyncaei, 1 (1935), 85-216.

[3] B. Hasselblatt, A. Katok, "The development of dynamics in the 20th century and the contribution of Jürgen Moser", Ergodic Theory Dynam. Systems, 22:5 (2002), 1343-1364.

[4] H. M. Morse, "Recurrent geodesics on a surface of negative curvature", Trans. Amer. Math. Soc., 22:1 (1921), 84-100.

[5] M. Morse, G. A. Hedlund, "Symbolic dynamics", Amer. J. Math., 60:4 (1938), $815-866$.

[6] M. Morse, G. A. Hedlund, "Symbolic dynamics. II. Sturmian trajectories", Amer. J. Math., 62:1 (1940), 1-42. 
[7] A. Thue, "Über unendliche Zeichenreihen", Norske Vidensk. Selsk. Skrifter. I. Mat. Nat. Kl., 7 (1906), 1-22; перепеч. в: Selected mathematical papers, Universitetsforlaget, Oslo, 1977, 139-158.

[8] A. Thue, "Über die gegenseitige Lage gleicher Teile gewisser Zeichenreihen", Norske Vidensk. Selsk. Skrifter. I. Mat. Nat. Kl., 1 (1912), 1-67; перепеч. в: Selected mathematical papers, Universitetsforlaget, Oslo, 1977, 413-478.

[9] С.И. Адян, Определяющие соотношения и алгоритмические проблемы для групn и полугрупn, Тр. МИАН, 85, Наука, М., 1966; англ. пер.: S. I. Adjan, Defining relations and algorithmic problems for groups and semigroups, Proc. Steklov Inst. Math., 85, Amer. Math. Soc., Providence, RI, 1966.

[10] А.Л. Семенов, "О некоторых расширениях арифметики сложения натуральных чисел”, Изв. АН СССР. Сер. матем., 43:5 (1979), 1175-1195; англ. пер.: A.L. Semenov, "On certain extensions of the arithmetic of addition of natural numbers", Math. USSR-Izv., 15:2 (1980), 401-418.

[11] А. Л. Семенов, “Логические теории одноместных функций на натуральном ряде”, Изв. АН СССР. Сер. матем., 47:3 (1983), 623-658; англ. пер.: A. L. Semenov, "Logical theories of one-place functions on the set of natural numbers", Math. USSR-Izv., 22:3 (1984), 587-618.

[12] Ю.Л. Притыкин, "Почти периодичность, конечно-автоматные преобразования и вопросы эффективности", Изв. вузов. Матем., 2010, № 1, 74-87; Almost periodicity, finite automata mappings and related effectiveness issues, arXiv: cs/0607009, 2006.

[13] М.А. Раскин, "Об оценке регулятора автоматного образа почти периодической последовательности", Труды XXVIII конферениии молодых ученых, Изд-во МГУ, М., 2006, 181-185.

[14] A. Yu. Rumyantsev, M. A. Ushakov, "Forbidden substrings, Kolmogorov complexity and almost periodic sequences", STACS"06, 23rd Annual Symposium on Theoretical Aspects of Computer Science (Marseile, 2006), Lecture Notes in Comput. Sci., 3884, ed. B. Durand et al., Springer, Berlin, 2006, 396-407.

[15] Колмогоровский семинар, http://kolmsem.math.ru.

[16] Семинар "бакторные языки", http://math.nsc.ru/seminar/lang/2009.html.

[17] J.-P. Allouche, J. Shallit, "The ubiquitous Prouhet-Thue-Morse sequence", Sequences and their applications (Singapore, 1998), Springer Ser. Discrete Math. Theor. Comput. Sci., Springer, London, 1999, 1-16.

[18] J. Berstel, D. Perrin, "The origins of combinatorics on words", European J. Combin., 28:3 (2007), 996-1022.

[19] J.-P. Allouche, A. Arnold, J. Berstel, S. Brlek, W. Jockusch, S. Plouffe, B. Sagan, "A relative of the Thue-Morse sequence", Formal power series and algebraic combinatorics (Montreal, PQ, 1992), Discrete Math., 139, № 1-3, 1995, 455-461.

[20] M. Euwe, "Mengentheoretische Betrachtungen über das Schachspiel", Proc. Konin. Akad. Wetenschappen, Amsterdam, 32 (1929), 633-642.

[21] M. Morse, "Abstract 360: a solution of the problem of infinite play in chess", Bull. Amer. Math. Soc., 44 (1938), 632.

[22] M. Morse, G. A. Hedlund, "Unending chess, symbolic dynamics and a problem in semi-groups", Duke Math. J., 11:1 (1944), 1-7.

[23] M.E. Prouhet, "Mémoire sur quelques relations entre les puissances des nombres", Comptes Rendus des Séances de l'Académie des Sciences, 33 (1851), 225; http:// gallica.bnf.fr/ark:/12148/bpt6k29901.image.f227.langFR.

[24] G. Tarry, "Question 4100", L'intermédiaire des mathématiciens, 19 (1912), 200.

[25] E. B. Escott, "Logarithmic series", Quart. J., 41:2 (1910), 141-156.

[26] E. B. Escott, "The calculation of logarithms", Quart. J., 41:2 (1910), 157-167. 
[27] G. H. Hardy, E. M. Wright, An introduction to the theory of numbers, 5th edition, The Clarendon Press, Oxford Univ. Press, New York, 1979.

[28] A. O. Gelfond, "Sur les nombres qui ont des propriétés additives et multiplicatives données", Acta Arith., 13 (1968), 259-265.

[29] C. Mauduit, J. Rivat, "Sur un problème de Gelfond: la somme des chiffres des nombres premiers", Ann. of Math. (to appear); http://pjm.math.berkeley.edu/ annals/ta/080511-Rivat/080511-Rivat-v1.pdf.

[30] П. С. Новиков, С. И. Адян, "О бесконечных периодических группах. I", Изв. АН СССР. Сер. матем., 32:1 (1968), 212-244; англ. пер.: P. S. Novikov, S. I. Adjan, "Infinite periodic groups. I", Math. USSR Izv., 2:1 (1968), 209-236.

[31] П. С. Новиков, С. И. Адян, "О бесконечных периодических группах. II", Изв. АН СССР. Сер. матем., 32:2 (1968), 251-524; англ. пер.: P. S. Novikov, S. I. Adjan, "Infinite periodic groups. II", Math. USSR Izv., 2:2 (1968), 241-479.

[32] П. С. Новиков, С. И. Адян, "О бесконечных периодических группах. III", Изв. АН СССР. Сер. матем., 32:3 (1968), 709-731; англ. пер.: P. S. Novikov, S. I. Adjan, "Infinite periodic groups. III", Math. USSR Izv., 2:3 (1968), 665-685.

[33] С.И. Адян, Проблема Бернсайда и тождества в группах, Наука, М., 1975; англ. пер.: S. I. Adian, The Burnside problem and identities in groups, Ergeb. Math. Grenzgeb., 95, Springer-Verlag, Berlin-New York, 1979.

[34] В. С. Кирсанов, "Уничтоженные книги: эхо сталинского террора в советской истории науки", Вопросы истории естествознания и техники, 2005, № 4, 105-124.

[35] Н. Я. Виленкин, "Формулы на фанере”, Природа, 6 (1991), 95-104.

[36] С. Е. Аршон, "Доказательство существования $n$-значных бесконечных асимметричных последовательностей”, Матем. просвещение, сер. 2, 2 (1934), 24-33.

[37] С. Е. Аршон, "Доказательство существования $n$-значных бесконечных асимметричных последовательностей", Матем. сб., 2:4 (1937), 769-779.

[38] M. Lothaire, Algebraic combinatorics on words, Encyclopedia Math. Appl., 90, Cambridge Univ. Press, Cambridge, 2002.

[39] J. Bernoulli, "Sur une nouvelle espèce de calcul", Recueil pour les astronomes, 1 (1775), 255-284.

[40] E. B. Christoffel, "Observatio arithmetica", Brioschi Ann. (2), 6:1 (1873), 148-153.

[41] A. Markoff, "Sur une question de Jean Bernoulli", Math. Ann., 19:1 (1881), 27-36.

[42] J.-P. Allouche, J. Shallit, Automatic sequences. Theory, applications, generalizations, Cambridge Univ. Press, Cambridge, 2003.

[43] H. Bohr, "Zur Theorie der fastperiodischen Funktionen. I", Acta Math., 45:1 (1925), 29-127.

[44] A.S. Besicovitch, Almost periodic functions, Cambridge Univ. Press, Cambridge, 1932.

[45] K. Jacobs, "Turing-Maschinen und zufallige 0-1-Folgen", Selecta Math., II, Heidelberger Taschenbucher, 67, Springer, Berlin, 1970, 141-167; пер. с англ.: К. Якобс, "Машины Тьюринга и рекурсивные функции", Популярная серия "Современная математика", ред. Г.-Д. Эббинхауз, К. Якобс, Ф.-К. Ман, Мир, М., 1972, 183-215.

[46] An. Muchnik, A. Semenov, M. Ushakov, "Almost periodic sequences", Theoret. Comput. Sci., 304:1-3 (2003), 1-33.

[47] Ю.Л. Притыкин, "Конечно-автоматные преобразования строго почти периодических последовательностей”, Матем. заметки, 80:5 (2006), 751-756; англ. пер.: Yu. L. Pritykin, "Finite-automaton transformations of strictly almost-periodic sequences", Math. Notes, 80:5-6 (2006), 710-714.

[48] M. Lothaire, Combinatorics on words, 2nd edition, Cambridge Math. Lib., Cambridge Univ. Press, Cambridge, 1997. 
[49] Ю.Л. Притыкин, "Конечно-автоматные преобразования почти периодических последовательностей и алгоритмическая неразрешимость", Труды XXVIII конференции молодых ученых, Изд-во МГУ, М., 2006, 177-181.

[50] J. Cassaigne, "Recurrence in infinite words", STACS (Dresden, 2001), Lecture Notes in Comput. Sci., 2010, Springer, Berlin, 2001, 1-11.

[51] N. P. Fogg, Substitutions in dynamics, arithmetics and combinatorics, Lecture Notes in Math., 1794, Springer-Verlag, Berlin, 2002.

[52] M. Keane, "Generalized Morse sequences", Z. Wahrscheinlichkeitstheor. verw. Geb., 10:4 (1968), 335-353.

[53] S. Kakutani, "Ergodic theory of shift transformations", Proceedings of the Fifth Berkeley Symposium on Mathematical Statistics and Probability. Vol. II: Contributions to Probability Theory, Part 2 (Berkeley, CA, 1965/66), Univ. California Press, Berkeley, CA, 1967, 405-414.

[54] A. Hoit, "Arithmetic on blocks", Integers, 4 (2004), A1.

[55] A. Cobham, "Uniform tag sequences", Math. Systems Theory, 6:1-2 (1972), 164-192.

[56] Yu. Pritykin, M. Raskin, Almost periodicity and finite automata, Electronic Proceedings of WIWAD (satellite to CSR 2007), Ekaterinburg, 2007.

[57] М. Раскин, "О произведении почти периодических последовательностей", не опубликовано.

[58] P. Salimov, "On uniform recurrence of a direct product", AutoMathA 2009 (Liège, Belgium, 2009); Discrete Math. Theor. Comput. Sci. (to appear).

[59] R. McNaughton, "Testing and generating infinite sequences by a finite automaton", Information and Control, 9:5 (1966), 521-530.

[60] J.R. Büchi, "On a decision method in restricted second-order arithmetic", Logic, Methodology and Philosophy of Science (1960), Stanford Univ. Press, Stanford, CA, 1962, 1-11.

[61] D. Siefkes, "Undecidable extensions of monadic second order successor arithmetic", Z. Math. Logik und Grundlagen der Math., 17:5 (1971), 383-394.

[62] O. Carton, W. Thomas, "The monadic theory of morphic infinite words and generalizations", Inform. and Comput., 176:1 (2002), 51-65.

[63] Х. Роджерс, Теория рекурсивных функиий и эффективная вычислимость, Мир, M., 1972; пер. с англ.: H. Rogers, Jr., Theory of recursive functions and effective computability, McGraw-Hill, New York-Toronto-London, 1967.

[64] Ю.Л. Притыкин, "О нерегулярности некоторых множеств бесконечных слов", Труды 30-й конференции молодых ученых ИТиС 2007, ИППИ РАН, Москва, 2007, 134-137.

[65] An. A. Muchnik, "The definable criterion for definability in Presburger arithmetic and its applications", Theoret. Comput. Sci., 290:3 (2003), 1433-1444.

[66] A. Adler, Sh.-Y.R. Li, "Magic cubes and Prouhet sequences", Amer. Math. Monthly, 84:8 (1977), 618-627.

[67] J.-P. Allouche, J. Shallit, "Sums of digits, overlaps, and palindromes", Discrete Math. Theor. Comput. Sci., 4:1 (2000), 1-10.

[68] P. Séébold, "On some generalizations of the Thue-Morse morphism", Theoret. Comput. Sci., 292:1 (2003), 283-298.

[69] Yu. Pritykin, "On almost periodicity criteria for morphic sequences in some particular cases", Developments in language theory, Lecture Notes in Comput. Sci., 4588, Springer, Berlin, 2007, 361-370.

[70] F. Nicolas, Yu. Pritykin, "On uniformly recurrent morphic sequences", Internat. J. Found. Comput. Sci., 20:5 (2009), 919-940.

[71] F.M. Dekking, "Iteration of maps by an automaton", Discrete Math., 126:1-3 (1994), 81-86. 
[72] J.-P. Allouche, "Sur la complexité des suites infinies", Journées Montoises (Mons, 1992), Bull. Belg. Math. Soc. Simon Stevin, 1, № 2, 1994, 133-143.

[73] S. Ferenczi, "Complexity of sequences and dynamical systems", Discrete Math., 206:1-3 (1999), 145-154.

[74] J. Cassaigne, "Sequences with grouped factors", Developments in language theory III (DLT'97), Aristotle University of Thessaloniki, 1998, 211-222.

[75] А.Л. Чернятьев, "Сбалансированные слова и динамические системы", Фундамент. и прикл. матем., 13:5 (2007), 213-224; англ. пер.: А. L. Chernyatiev, "Balanced words and dynamical systems", J. Math. Sci. (N. Y.), 156:2 (2009), 351-358.

[76] J.-J. Pansiot, "Complexité des facteurs des mots infinis engendrés par morphismes itérés", Automata, languages and programming (Antwerp, 1984), Lecture Notes in Comput. Sci., 172, Springer, Berlin, 1984, 380-389.

[77] J.-J. Pansiot, "Subword complexities and iteration", Bull. Eur. Assoc. Theor. Comput. Sci. EATCS, 26 (1985), 55-62.

[78] R. Devyatov, "On subword complexity of morphic sequences", Computer science theory and applications, Lecture Notes in Comput. Sci., 5010, Springer, Berlin, 2008, 146-157.

[79] R. Devyatov, "More on subword complexity of morphic sequences" (to appear).

[80] S. V. Avgustinovich, D. G. Fon-Der-Flaass, A. E. Frid, "Arithmetical complexity of infinite words", Words, languages \& combinatorics, III (Kyoto, 2000), eds. M. Ito, T. Imaoka, World Sci. Publ., River Edge, NJ, 2003, 51-62.

[81] A. E. Frid, "On possible growths of arithmetical complexity", Theor. Inform. Appl., 40:3 (2006), 443-458.

[82] S. V. Avgustinovich, J. Cassaigne, A. E. Frid, "Sequences of low arithmetical complexity", Theor. Inform. Appl., 40:4 (2006), 569-582.

[83] M. Waldschmidt, "Words and transcendence", Analytic number theory, Cambridge Univ. Press, Cambridge, 2009, 449-470.

[84] É. Borel, "Les probabilités dénombrables et leurs applications arithmétiques", Rend. Circ. Mat. Palermo (2), 27:1 (1909), 247-271.

[85] É. Borel, "Sur les chiffres décimaux de $\sqrt{2}$ et divers problèmes de probabilités en chaînes", C. R. Acad. Sci. Paris, 230 (1950), 591-593.

[86] S. Ferenczi, C. Mauduit, "Transcendence of numbers with a low complexity expansion", J. Number Theory, 67:2 (1997), 146-161.

[87] B. Adamczewski, Y. Bugeaud, "On the complexity of algebraic numbers. I. Expansions in integer bases", Ann. of Math. (2), 165:2 (2007), 547-565.

[88] A. Cobham, "On the Hartmanis-Stearns problem for a class of tag machines", Proceedings of the 9th Annual Symposium on Switching and Automata Theory (SWAT'68), IEEE Computer Society, 1968, 51-60.

[89] J. Albert, Propriétés combinatoires et arithmétiques de certaines suites automatiques et substitutives, Thèse de Doctorat, Université Paris XI, Orsay, 2006.

[90] Y. Bugeaud, J.-H. Evertse, "On two notions of complexity of algebraic numbers", Acta Arith., 133:3 (2008), 221-250.

[91] M. Li, P. Vitányi, An introduction to Kolmogorov complexity and its applications, 2nd edition, Grad. Texts Comput. Sci., Springer-Verlag, New York, 1997.

[92] В. А. Успенский, Н. К. Верещагин, А. Шень, Колмогоровская сложность, Неопубл. книга; http://kolmsem.math.ru/rus/materials/materials.html.

[93] Ю. Л. Притыкин, "Колмогоровская сложность", Матем. просвещение, сер. 3, 13 (2009), 127-133.

[94] A. Yu. Rumyantsev, "Kolmogorov complexity, Lovász local lemma and critical exponents", Proceedings of the 2 nd International Symposium on Computer Science in 
Russia (CSR 2007, Ekaterinburg), Lecture Notes in Comput. Sci., 4649, Springer, Berlin, 2007, 349-355.

[95] D. Krieger, J. Shallit, "Every real number greater than 1 is a critical exponent", Theoret. Comput. Sci., 381:1-3 (2007), 177-182.

[96] Yu. Pritykin, "Information in infinite words", Proceedings of the 6th International Conference on Words (WORDS 2007, CIRM, Marseille, France), 2007, 254-261.

[97] B. Durand, L. Levin, A. Shen, "Complex tilings", Proceedings of the Thirty-Third Annual ACM Symposium on Theory of Computing, ACM, New York, 2001, 732-739; arXiv: cs/0107008, 2001.

[98] M.-P. Béal, D. Perrin, "Symbolic dynamics and finite automata", Handbook of formal languages, vol. 2, eds. G. Rozenberg, A. Salomaa, Springer, Berlin, 1997, 463-505.

[99] Yu. Pritykin, J. Ulyashkina, "Aperiodicity measure for infinite sequences", Proceedings of CSR 2009 (Novosibirsk, 2009), Lecture Notes in Comput. Sci., 5675, Springer, Berlin, 2009, 274-285.

[100] B. Durand, A. Romashchenko, A. Shen, "Fixed point and aperiodic tilings", Proceedings of DLT 2008, Lecture Notes in Comput. Sci., 5257, Springer, Berlin, 2008, 276-288; arXiv: 0802.2432.

[101] E. Grant, J. Shallit, T. Stoll, "Bounds for the discrete correlation of infinite sequences on $k$ symbols and generalized Rudin-Shapiro sequences", Acta Arith. (to appear); arXiv: 0812.3186, 2008.

[102] J. Cassaigne, N. Chekhova, "Fonctions de récurrence des suites d'Arnoux-Rauzy et réponse à une question de Morse et Hedlund", Ann. Inst. Fourier (Grenoble), 56:7 (2006), 2249-2270.

[103] G. Rauzy, "Suites à termes dans un alphabet fini", Seminar on number theory, Exp. № 25 (Talence, 1982/1983), Univ. Bordeaux I, Talence, 1983.

[104] A. Apostolico, A. Ehrenfeucht, "Efficient detection of quasiperiodicities in strings", Theoret. Comput. Sci., 119:2 (1993), 247-265.

[105] S. Marcus, "Quasiperiodic infinite words", Bull. Eur. Assoc. Theor. Comput. Sci. EATCS, 82 (2004), 170-174.

[106] F. Levé, G. Richomme, "Quasiperiodic infinite words: some anwers", Bull. Eur. Assoc. Theor. Comput. Sci. EATCS, 84 (2004), 128-138.

[107] T. Monteil, S. Marcus, Quasiperiodic infinite words: multi-scale case and dynamical properties, arXiv: math/0603354, 2006.

[108] F. Levé, G. Richomme, "Quasiperiodic Sturmian words and morphisms", Theoret. Comput. Sci., 372:1 (2007), 15-25; arXiv: cs/0601027, 2006.

[109] J. Karhumäki, Y. Lifshits, W. Rytter, "Tiling periodicity", Proceedings of CPM 2007, Lecture Notes in Comput. Sci., 4580, Springer, Berlin, 2007, 295-306.

[110] D. J. Newman, "Tesselation of integers", J. Number Theory, 9:1 (1977), 107-111.

[111] O. Toeplitz, "Ein Beispiel zur Theorie der fastperiodischen Funktionen", Math. Ann., 98:1 (1928), 281-295.

[112] K. Jacobs, M. Keane, "0-1-sequences of Toeplitz type", Z. Wahrscheinlichkeitstheor. verw. Geb., 13:2 (1969), 123-131.

[113] J. Cassaigne, J. Karhumäki, "Toeplitz words, generalized periodicity and periodically iterated morphisms", European J. Combin., 18:5 (1997), 497-510.

[114] K. Culik II, J. Karhumäki, "Iterative devices generating infinite words", Proceedings of STACS'92, Lecture Notes in Comput. Sci., 577, Springer, Berlin, 1992, 529-543.

[115] A. Lepistö, "On the power of periodic iteration of morphisms", Automata, languages and programming (Lund, 1993), Lecture Notes in Comput. Sci., 700, Springer, Berlin, 1993, 496-506.

[116] W. Kolakoski, "Problem 5304: Self generating runs", Amer. Math. Monthly, 72:6 (1965), 674. 
[117] W. Kolakoski, N. Ucoluk, "Problems and solutions: Solutions of advanced problems: 5304", Amer. Math. Monthly, 73:6 (1966), 681-682.

[118] E.W. Weisstein, Kolakoski sequence, http://mathworld.wolfram.com/KolakoskiSequence.html.

[119] A. Scolnicov, PlanetMath: Kolakoski Sequence, http://planetmath.org/encyclopedia/KolakoskiSequence.html.

[120] C. Kimberling, Integer sequences and arrays, http://faculty.evansville.edu/ ck6/integer/.

[121] C. Kimberling, Unsolved problems and rewards, http://faculty.evansville.edu/ ck6/integer/unsolved.html.

[122] N.J.A. Sloane, On-line encyclopedia of integer sequences, id:A000002, http:// www. research.att.com/ njas/sequences/A000002.

[123] K. Culik II, J. Karhumäki, A. Lepistö, "Alternating iteration of morphisms and the Kolakovski sequence", Lindermayer systems, eds. G. Rozenberg, A. Salomaa, Springer, Berlin, 1992, 93-106.

[124] С.И. Адян, А.Л. Семенов, В.А. Успенский, "Андрей Альбертович Мучник (некролог)", УМH, 62:4 (2007), 140-144; англ. пер.: S. I. Adian, A. L. Semenov, V. A. Uspenskii, "Andrei Al'bertovich Muchnik (obituary)", Russian Math. Surveys, 62:4 (2007), 775-779.

Ан. А. Мучник (An. A. Muchnik)

Институт новых технологий образования, Москва

Ю. Л. Притыкин (Yu. L. Pritykin)

Московский государственный университет

им. М. В. Ломоносова

E-mail: pritykin@lpcs.math.msu.su, yuri.pritykin@gmail.com

\section{А. Л. Семенов (А. L. Semenov)}

Вычислительный центр им. А. А. Дородницына РАН

E-mail: alsemenov@mtu-net.ru, alsemenov@umail.ru
Поступила в редакцию

07.08.2009 